\title{
THE SPITZER c2d SURVEY OF LARGE, NEARBY, INTERSTELLAR CLOUDS. X. THE CHAMAELEON II PRE-MAIN-SEQUENCE POPULATION AS OBSERVED WITH IRAC AND MIPS
}

\author{
Juan M. Alcalá, ${ }^{1}$ Loredana Spezzi, ${ }^{1,2}$ Nicholas Chapman, ${ }^{3}$ Neal J. Evans II, ${ }^{4}$ Tracy L. Huard, \\ Jes K. Jørgensen, ${ }^{6}$ Bruno Merín, ${ }^{7,8}$ Karl R. Stapelfeldt, ${ }^{9}$ Elvira Covino, ${ }^{1}$ \\ Antonio Frasca, ${ }^{2}$ Davide Gandolfi, ${ }^{2}$ and Isa Oliveira ${ }^{8,10}$ \\ Received 2007 August 28; accepted 2007 November 17
}

\begin{abstract}
We discuss the results from the combined IRAC and MIPS c2d Spitzer Legacy survey observations and complementary optical and NIR data of the Chamaeleon II (Cha II) dark cloud. We perform a census of the young population in an area of $\sim 1.75 \mathrm{deg}^{2}$ and study the spatial distribution and properties of the cloud members and candidate premain-sequence (PMS) objects and their circumstellar matter. Our census is complete down to the substellar regime $\left(M \approx 0.03 M_{\odot}\right)$. From the analysis of the volume density of the PMS objects and candidates we find two groups of objects with volume densities higher than $25 M_{\odot} \mathrm{pc}^{-3}$ and 5-10 members each. A multiplicity fraction of about $13 \% \pm 3 \%$ is observed for objects with separations $0.8^{\prime \prime}<\theta<6.0^{\prime \prime}(142-1065 \mathrm{AU})$. No evidence for variability between the two epochs of the c2d IRAC data set, $\Delta t \sim 6 \mathrm{hr}$, is detected. We estimate a star formation efficiency of $1 \%-4 \%$, consistent with the estimates for Taurus and Lupus, but lower than for Cha I. This might mean that different star formation activities in the Chamaeleon clouds reflect a different history of star formation. We also find that Cha II is turning some 6-7 $M_{\odot}$ into stars every Myr, which is low in comparison with the star formation rate in other c2d clouds. The disk fraction of $70 \%-80 \%$ that we estimate in Cha II is much higher than in other star-forming regions and indicates that the population in this cloud is dominated by objects with active accretion. Finally, the Cha II outflows are discussed; a new Herbig-Haro outflow, HH 939, driven by the classical T Tauri star Sz 50, has been discovered.
\end{abstract}

Subject headings: circumstellar matter — infrared: stars — planetary systems: protoplanetary disks — stars: formation — stars: low-mass, brown dwarfs — stars: pre-main-sequence

Online material: color figures

\section{INTRODUCTION}

The Chamaeleon cloud complex is one of the major starforming regions within $200 \mathrm{pc}$ from the Sun. It consists of three main dark clouds, namely, Cha I, Cha II, and Cha III (Schwartz 1991). In terms of star formation, the most active of the three is Cha I, while Cha III is the least active one (Schwartz 1977; Gregorio Hetem et al. 1988).

At a distance of $178 \mathrm{pc}$ (Whittet et al. 1997), the Cha II dark $\operatorname{cloud}\left(\right.$ R.A. $\approx 13^{\mathrm{h}} 00^{\mathrm{m}}$ and decl. $\approx-77^{\circ} 06^{\prime}$ ) is a well-defined structure that covers about $2 \mathrm{deg}^{2}$ in the sky. The presence of pre-main-sequence (PMS) objects in this cloud has been traced by a variety of observations, including objective prism surveys (Schwartz 1977; Hartigan 1993), X-ray imaging with ROSAT (Alcalá et al. 2000 and references therein), and infrared (IR) surveys with IRAS (Whittet et al. 1991; Prusti et al. 1992; Larson et al. 1998) and the Infrared Space Observatory (ISO; Persi et al.

\footnotetext{
1 INAF-OA Capodimonte, 80131 Naples, Italy; alcala@oacn.inaf.it.

2 INAF-OA Catania, 95123 Catania, Italy; afr@oact.inaf.it.

3 Astronomy Department, University of Maryland, College Park, MD 20742; chapman@astro.umd.edu.

4 Astronomy Department, University of Texas, Austin, TX 78712-0259; nje@ astro.as.utexas.edu

5 Smithsonian Astrophysical Observatory, Cambridge, MA 02138; thuard@ cfa.harvard.edu.

${ }^{6}$ Argelander-Institut für Astronomie, University of Bonn, 53121 Bonn, Germany; jes@astro.uni-bonn.de.

7 Research and Scientific Support Department (ESTEC), European Space Agency, 2200 AG Noordwijk, Netherlands; bmerin@rssd.esa.int.

8 Leiden Observatory, Leiden University, 2300 RA Leiden, Netherlands.

9 Jet Propulsion Laboratory, California Institute of Technology, Pasadena, CA 91109; krs@exoplanet.jpl.nasa.gov.

10 Division of Geological and Planetary Sciences, California Institute of Technology, Pasadena, CA 91125; isa@gps.caltech.edu.
}

2003). Some 20 classical T Tauri stars (CTTSs) and a number of weak-line T Tauri star (WTTS) candidates have been identified in the cloud so far from ground-based optical and near-IR (NIR) photometry and subsequent spectroscopic follow-up (Hughes \& Hartigan 1992; Alcalá et al. 1995; Covino et al. 1997a; Larson et al. 1998; Vuong et al. 2001; Barrado y Navascués \& Jayawardhana 2004; Allers et al. 2007). In addition to the low-mass young stars, one intermediate-mass AeBe star, IRAS 12496-7650, and the well-studied Herbig-Haro object HH 54 (Knee 1992; Liseau et al. 1996; Nisini et al. 1996; Neufeld et al. 1998, 2006) are also associated with Cha II.

The Cha II dark cloud is one of the five star formation regions selected by the Legacy program "From Molecular Cores to Planet-forming Disks" (c2d; Evans et al. 2003). Cha II was chosen for the $\mathrm{c} 2 \mathrm{~d}$ program as an example of a cloud with modest star formation activity, based on previous data. One of the major goals was to investigate whether the modest star formation activity in the cloud is the result of observational biases and the more sensitive data from Spitzer would discover a more vigorous star formation, or whether the modest star formation is real. For instance, previous ground-based studies (Barrado y Navascués \& Jayawardhana 2004) failed to find young substellar objects. Hence, an important goal of the $\mathrm{c} 2 \mathrm{~d}$ program has been to confirm whether the nondetection of brown dwarfs (BDs) in Cha II is due to incompleteness of the previous surveys, in both sky area and flux, or to a real lack of BDs in the region. Recent follow-up of the $\mathrm{c} 2 \mathrm{~d}$ observations has now confirmed the substellar nature of some objects in Cha II (Allers et al. 2006, 2007; Alcalá et al. 2006; Jayawardhana \& Ivanov 2006; Spezzi et al. 2007b, hereafter S07b).

The Spitzer observations in Cha II are reported in previous basic data papers of this series: the MIPS (Multiband Imaging Photometer for Spitzer) observations are presented in Young et al. 
(2005), while those with IRAC (Infrared Array Camera) are reported in Porras et al. (2007). Young et al. (2005) also presented a $1.2 \mathrm{deg}^{2}$ millimeter map of Cha II obtained with SIMBA on the Swedish-ESO Submillimetre Telescope (SEST); they show that only a small fraction of the gas is in compact structures with high column density. Complementary to the $\mathrm{c} 2 \mathrm{~d}$ survey, data in Cha II in optical and NIR wavelengths are presented in Spezzi et al. (2007a, hereafter S07a) and Allers et al. (2006), respectively.

The main goal of this paper is to combine the c2d IRAC and MIPS data with the complementary data, and with other data existing in the literature, to make a synthesis of the young population of Cha II. Our intention is to deliver the most reliable census to date of the young population that permits a thorough study of the star formation in this cloud. To this aim, we report the available photometry in the range of $0.36-1300 \mu \mathrm{m}$ for as many Cha II members as possible. The IRAC observations reported in Porras et al. (2007) were performed with the criterion to cover the area with visual extinction greater than $2 \mathrm{mag}$. The consequence of this is that only the central and "densest" part of the Cha II cloud was investigated with IRAC. Thus, to perform a complete census, we also include the PMS objects and candidates that lie off the cloud, in regions where no IRAC observations were performed. The data presented here are used to investigate the spectral energy distribution (SED) of the Cha II certified and candidate PMS objects and study their circumstellar disk/envelope properties.

Throughout this paper we use the terms young stellar object (YSO) and PMS object. The c2d convention is to define the former as those PMS objects with detectable IR excess in at least one of the Spitzer bands and that satisfy the multicolor criteria described in Harvey et al. (2007, hereafter H07) and Evans et al. (2007), while the latter considers a broader class that includes not only YSOs but also PMS objects discovered using other observational techniques like $\mathrm{H} \alpha$ and $\mathrm{X}$-ray surveys or chromospheric activity indicators. Thus, by PMS objects we mean objects not yet on the main sequence that may or may not have IR excess. That is, all YSOs are PMS objects, but not vice versa. Therefore, in this paper the term YSO includes all the envelope and disk stages as a subset of all PMS phases.

In $\S 2$ we present an overview on the observational photometric data set used, while in $\S 3$ the census of the PMS population in Cha II is performed by merging the $\mathrm{c} 2 \mathrm{~d}$ data with those from optical and IR surveys. In $\S 4$ a search for variability in the c $2 d$ data set is described. In $\S 5$ the photometric data set is used to investigate the global properties of the circumstellar material, including the comparison with accretion models in color-color diagrams, as well as from fits of both accretion and reprocessing models to the SEDs. The disk frequency and disk properties versus stellar parameters are also discussed in this section. In $\S 6$ the results on the efficiency and rate of star formation in the cloud are discussed, making use of the extinction maps so far derived in Cha II to estimate the cloud mass. Finally, in $\S 7$ we give an overview of the outflows in Cha II and report the discovery of new Herbig-Haro objects. We summarize our results in $\S 8$.

\section{PHOTOMETRIC DATA SET}

The basic data set used for this paper comes from the Spitzer (IRAC+MIPS) c2d observations of Cha II, complemented with optical and NIR imaging surveys.

In addition, data from the Spitzer Wide-area Infrared Extragalactic $\left(\mathrm{SWIRE}^{11}\right)$ survey are used for statistical analysis and corrections for background contaminants.

\subsection{Spitzer Data}

The observations, data reduction, source statistics, and the results from the IRAC observations are reported in Porras et al. (2007). An area of $1.04 \mathrm{deg}^{2}$ in Cha II was mapped in the four IRAC bands $(3.6,4.5,5.8$, and $8 \mu \mathrm{m})$ with the criterion that $A_{V}>2 \mathrm{mag}$ in the extinction map reported by Cambrésy (1999). In order to make statistical comparisons of source counts and determine the contamination by background objects, six off-cloud fields were also observed with IRAC adopting the criterion of $A_{V}<0.5$ mag in the Cambrésy (1999) map. Based on the 2005 catalog, some 40 sources were classified as YSO candidates from the IRAC observations by Porras et al. (2007). However, as pointed out in that paper, a significant fraction of these candidates may correspond to extragalactic contaminants. This issue is discussed in more detail in $\S 3$.

The results of the MIPS survey in Cha II are presented in Young et al. (2005). The surveyed area of $1.5 \mathrm{deg}^{2}$ entirely covers the one mapped with IRAC. By combining the MIPS data with those of the Two Micron All Sky Survey (2MASS) catalog (Cutri et al. $2003^{12}$ ) and using color-color and color-magnitude diagrams, Young et al. (2005) classified more than 40 sources as potential YSO candidates. Considering the completeness in area and flux, and despite the low number of potential YSO candidates, Young et al. (2005) concluded that the population of YSO candidates found in previous surveys is nearly complete.

An important step beyond the $2005 \mathrm{c} 2 \mathrm{~d}$ catalogs was the creation of new "band-filled" catalogs. Such band filling was performed by the $\mathrm{c} 2 \mathrm{~d}$ catalog infrastructure in order to obtain upper limits or low signal-to-noise $(\mathrm{S} / \mathrm{N})$ detections of objects that were not detected in the original source extraction processing. Details on the band-filling process, which is applied only to sources clearly detected in at least one of the Spitzer bands, are described in the final $\mathrm{c} 2 \mathrm{~d}$ data delivery document (Evans et al. 2007). For a short description we refer the reader to H07.

\subsection{Complementary Optical to Millimeter Data}

In the following sections we describe the complementary data used for this work.

The optical imaging survey was performed using the Wide Field Imager (WFI) attached to the ESO $2.2 \mathrm{~m}$ telescope, at La Silla, Chile. The observational strategy, data reduction, and source extraction are described in S07a. An area of about $1.75 \mathrm{deg}^{2}$ in Cha II was observed in the $R_{\mathrm{C}}, I_{\mathrm{C}}, z$, and $\mathrm{H} \alpha$ bands and in two intermediate-band filters. The optical observations cover the overlap areas observed with IRAC and MIPS. By combining the optical data with those of 2MASS and using color-magnitude and color-color diagrams, S07a selected 10 new candidate PMS objects not revealed by previous surveys. These photometric data also confirmed the $\mathrm{H} \alpha$ emission from these objects. They concluded that these objects are most likely Class III sources belonging to the WTTS class, as expected by the complementary character of the optical survey with respect to the IR surveys. In line with Young et al. (2005), S07a concluded that the optical surveys performed so far in Cha II may have already provided a population of PMS objects that is basically complete.

In addition to the optical survey, a deep NIR survey was performed by Allers et al. (2006) over an area of about $0.6 \mathrm{deg}^{2}$ in Cha II. Based on color-magnitude and color-color diagrams, they selected seven candidate very low mass PMS objects in Cha II. Three of them were confirmed to be young substellar objects based on follow-up spectroscopy (Alcalá et al. 2006; Jayawardhana \& 
Ivanov 2006; Allers et al. 2007), among these the lowest mass objects in Cha II (Allers et al. 2007).

Photometric data from the "Naval Observatory Merged Astrometric Dataset" (NOMAD; Zacharias et al. 2004) in the optical and from the $I S O$ observations by Persi et al. (2003) in the IR and by Henning et al. (1993) in millimeter wavelengths are used as ancillary data for this work. In particular, these data are incorporated in the SEDs presented in $\S 5.2$.

Finally, the spectral types and physical parameters of the PMS objects and candidates in Cha II derived by S07b on the basis of optical spectroscopic follow-ups are used here for the determination and analysis of the SEDs, as well as for the analysis of the overall star formation in Cha II.

\subsection{SWIRE Data}

In order to perform statistical comparisons and correct for extragalactic contaminants, we also use in this work the much larger and deeper catalog coming from the SWIRE observations (see footnote 11) of the ELAIS N1 field (Rowan-Robinson et al. 2004). The SWIRE catalog was extincted and resampled as accurately as possible to match the $\mathrm{c} 2 \mathrm{~d}$ sensitivity limits, as well as extinction in Cha II. For the details on how the trimmed resampled SWIRE comparison catalog was created, we refer the reader to the final c2d data delivery document (Evans et al. 2007) and for a short description to $\mathrm{H} 07$.

\section{THE SAMPLE OF PMS OBJECTS AND CANDIDATES IN CHA II}

The IRAC observations in Cha II cover only the central and "densest" part of the cloud. However, some previously known members and candidate PMS objects lie to the east of the Cha II cloud (see Fig. 9 below). Thus, in order to include these objects, our census is performed by merging the $\mathrm{c} 2 \mathrm{~d}$ data with those from previous optical and NIR surveys. In the following we describe how our list of certified and candidate members of Cha II was constructed. The IRAC and MIPS data are used first to select new YSO candidates and to estimate their number in regions where no IRAC observations were performed. The certified and candidate PMS objects without IRAC observations are then discussed.

The samples of PMS objects and candidates presented in the previous surveys plus the sources selected from new c $2 \mathrm{~d}$ criteria (see $\S 3.2$ ) in this work were merged into a single list, which is given in Table 1. A spectroscopic follow-up of many of the candidate PMS objects has been performed with FLAMES at the ESO VLT, but the details and results of these observations are published in a companion paper (S07b). From such investigation we conclude that there are 51 certified PMS objects plus 11 candidates that still need spectroscopic investigation in order to firmly assess their nature. Of these 62 objects, 26 are YSOs selected from the $\mathrm{c} 2 \mathrm{~d}$ criteria; 2 of these 26 were previously unknown, while the remaining 24 coincide with Cha II members or candidate PMS objects identified in early surveys. The sample is flagged in the fifth column of Table 1 with "PMS" indicating a certified PMS object, with "CND" a candidate PMS object, and with "YSO" if the object passed the new c2d selection criteria described in $\S 3.1$. If the young nature of the 11 candidates will be confirmed, the population of PMS objects in Cha II will increase to more than 60 members.

In Table 1 we also report the sources HH 54 (or IRAS 125227640) and IRAS 13036-7644. The former corresponds to the head of the HH 54 outflow (see $\S 7$ ), while the latter, also known as BHR 86 (Bourke et al. 1995), has been reported by Lehtinen et al. (2005) to be a transition object between Class 0 and I. BHR 86 is located to the east, somewhat detached from the main body of the Cha II cloud. BHR 86 is a matter of focused c $2 \mathrm{~d}$ observations and will be studied in more detail in a future paper (T. L. Huard et al. 2008, in preparation).

The Spitzer fluxes were extracted from the new band-filled catalog described in $\S 2.1$. These, in combination with the optical magnitudes reported by S07a and those at longer wavelengths available in the literature for some objects, constitute the main data set for our subsequent analysis. In Tables 2, 3, 4, and 5 we report the available photometric data for the sources reported in Table 1.

We remind the reader that the IRAC observations were performed in such a way that the coverage at 3.6 and $5.8 \mu \mathrm{m}$ is shifted by about $7^{\prime}$ to the east of the area covered at 4.5 and $8.0 \mu \mathrm{m}$ (see Fig. 1 by Porras et al. 2007). Thus, some sources possessing fluxes at 3.6 and $5.8 \mu \mathrm{m}$ may lack data at 4.5 and $8.0 \mu \mathrm{m}$, and vice versa. In the eighth column of Table 1, the flag "In" is used to indicate that the object lies on the IRAC 3.6-8 $\mu \mathrm{m}$ /MIPS $24 \mu \mathrm{m}$ overlap area; the flag "Off" is used otherwise.

\subsection{Selection of YSOs}

One of the main goals of the c2d observations was the selection of new YSO candidates based on their IR emission. However, the IR colors of many galaxies are very similar to those of YSOs. Thus, a sample of YSO candidates must be corrected for the contamination of background extragalactic objects. This and the next subsection explain how the 26 YSOs were selected.

The first criteria for the selection of YSO candidates from the Spitzer data are described in the third delivery document of the c2d program (Evans et al. 2005). These criteria, applied to the IRAC data of the 2005 catalog, provided $\sim 40$ YSO candidates in Cha II with a density of 38.5 candidates $\mathrm{deg}^{-2}$ (Porras et al. 2007). Interestingly, many Class I or flat-spectrum sources were identified among these candidates, indicating a much more active star formation in Cha II than previously found. It is also interesting to note that, despite the larger area covered with MIPS, Young et al. (2005) also selected 44 YSO candidates, but with the smaller density of 29.3 objects deg $^{-2}$. This cast doubts on the YSO nature of the new Class I or flat-spectrum sources. As pointed out by Porras et al. (2007), a substantial fraction of the low-luminosity objects, classified as Class I or flat-spectrum sources from the IRAC data, may correspond to extragalactic contaminants. We now provide further arguments supporting this conclusion.

Figure 1 shows the four luminosity histograms corresponding to the Lada classes as determined by Porras et al. (2007). The luminosities were computed by integrating the SEDs, from 1.2 to $70 \mu \mathrm{m}$, using the available fluxes for each source. The dashed histograms represent the total sample of YSO candidates, while the solid ones represent only those selected from new criteria described below. In the dashed histograms, low luminosity is much more common for earlier Lada classes. The few more luminous sources in this sample are mostly Class III, well-known stars with enough circumstellar dust to be luminous, but not very embedded. Essentially all the Class I and flat-spectrum sources have a luminosity of less than $0.01 L_{\odot}$. All these ultra-low-luminosity sources, with not much to be embedded in given the SIMBA millimeter map reported in Young et al. (2005), are suspected to be background galaxies.

Additional arguments indicating that the low-luminosity sources are extragalactic contaminants come from their appearance in the optical images. Figure 2 shows $I$-band images of such objects collected in the optical survey by S07a. Although some sources are barely seen, the vast majority of them clearly appear as extended, most likely corresponding to extragalactic contaminants. 
TABLE 1

Pre-Main-Sequence Objects and Candidates in Cha II

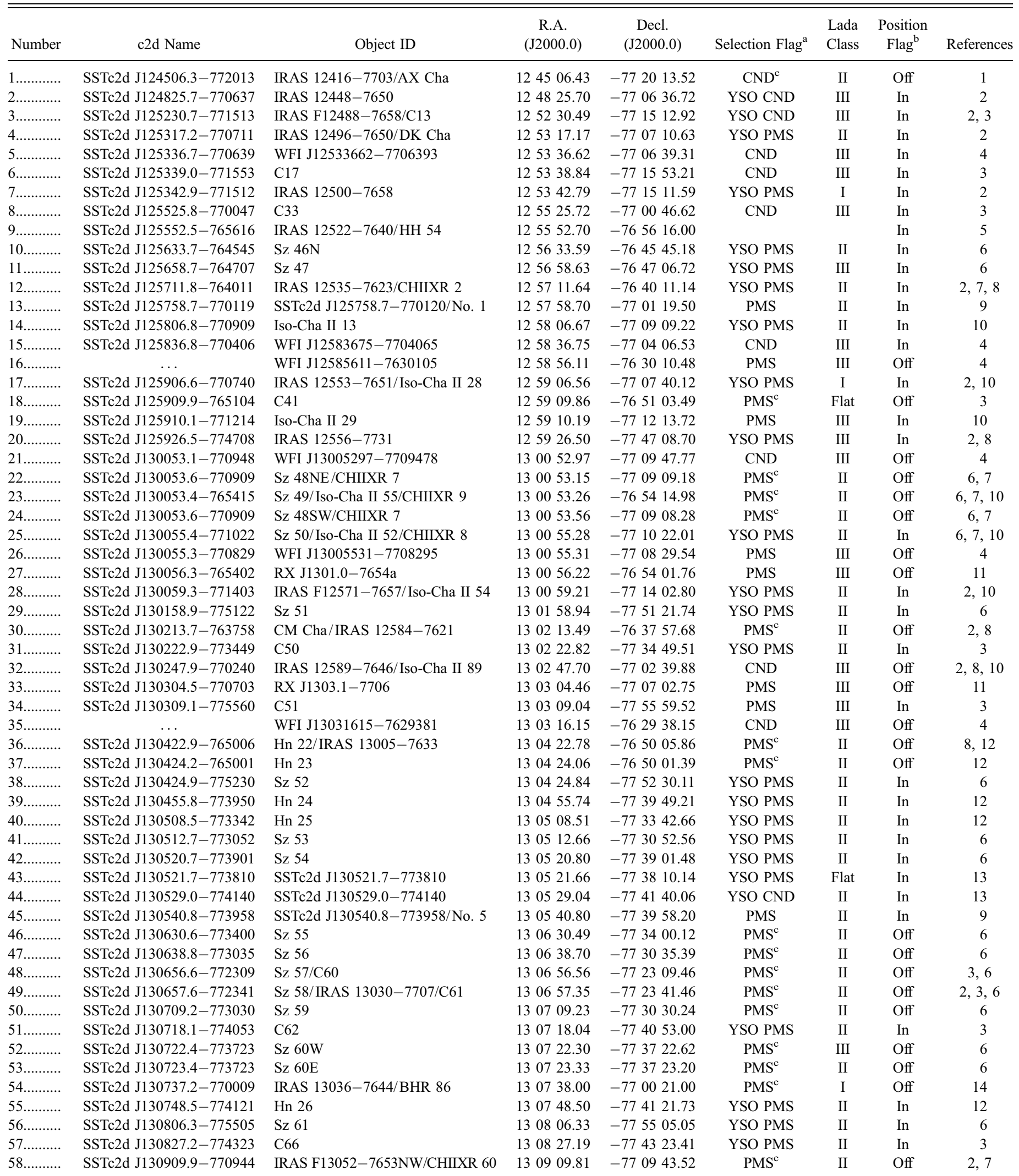


TABLE 1 - Continued

\begin{tabular}{|c|c|c|c|c|c|c|c|c|}
\hline Number & c2d Name & Object ID & $\begin{array}{c}\text { R.A. } \\
\text { (J2000.0) }\end{array}$ & $\begin{array}{c}\text { Decl. } \\
\text { (J2000.0) }\end{array}$ & Selection Flag ${ }^{\mathrm{a}}$ & $\begin{array}{l}\text { Lada } \\
\text { Class }\end{array}$ & $\begin{array}{l}\text { Position } \\
\text { Flag }^{\mathrm{b}}\end{array}$ & References \\
\hline $59 \ldots \ldots \ldots . . .$. & SSTc2d J130910.7-770944 & IRAS F13052-7653N/CHIIXR 60 & 130910.98 & -770944.14 & $\mathrm{PMS}^{\mathrm{c}}$ & II & Off & 2,7 \\
\hline $61 \ldots \ldots \ldots . . .$. & SSTc2d J131004.2-771045 & $\mathrm{Sz} 63$ & 131004.12 & -771044.62 & $\mathrm{PMS}^{\mathrm{c}}$ & II & Off & 6 \\
\hline $62 \ldots \ldots \ldots \ldots$ & SSTc2d J131252.4-773918 & 2MASS $13125238-7739182$ & 131252.37 & -773918.58 & PMS & III & Off & 15 \\
\hline $63 \ldots \ldots \ldots \ldots$ & SSTc2d J131403.8-775308 & Sz 64 & 131403.83 & -775307.48 & $\mathrm{PMS}^{\mathrm{c}}$ & II & Off & 6 \\
\hline
\end{tabular}

NoтES.-Most coordinates are from optical images, where the multiple objects are better resolved. Units of right ascension are hours, minutes, and seconds, and units of declination are degrees, arcminutes, and arcseconds. The c2d names are as in the c2d final delivery document by Evans et al. (2007). We note that, because of reprocessing during the source extraction for new catalog releases, some of these names may be slightly different than those reported in Young et al. (2005) for the same sources.

${ }^{\mathrm{a}}$ PMS $=$ pre-main-sequence object; CND $=$ candidate pre-main-sequence object; YSO = object with IRAC/MIPS $24 \mu \mathrm{m}$ colors that passed the H07 criteria IRAS $12496-7650$ is definitely a YSO, but not selected by the H07 criteria because it is saturated in IRAC $3.6 \mu \mathrm{m}$ and IRAC $4.5 \mu \mathrm{m}$.

${ }^{\mathrm{b}}$ In = object inside the IRAC 3.6-8 $\mu \mathrm{m}$ /MIPS $24 \mu \mathrm{m}$ overlapping area; Off = object off the IRAC $3.6-8 \mu \mathrm{m} / \mathrm{MIPS} 24 \mu \mathrm{m}$ overlapping area.

${ }^{c}$ Would have met the c2d selection criteria if IRAC observations had been available.

References.-(1) Gauvin \& Strom 1992; (2) Prusti et al. 1992; (3) Vuong et al. 2001; (4) S07a; (5) Knee 1992; (6) Schwartz 1991; (7) Alcalá et al. 2000; (8) Larson et al. 1998; (9) Allers et al. 2006; (10) Persi et al. 2003; (11) Alcalá et al. 1995; (12) Hartigan 1993; (13) this paper; (14) Bourke et al. 1995; (15) Young et al. 2005.

Four sources, namely, SSTc2d J125944.9-774808, SSTc2d 130201.3-774813, SSTc2d 130217.0-774121, and SSTc2d $130400.9-774811$, seem to present a more pointlike PSF than the others, but none of them have been selected as candidate PMS objects in previous surveys, nor do they appear as $\mathrm{H} \alpha$ emitters (see S07a). In addition, the IRAC fluxes of two of them (SSTc2d 125944.9-774808 and SSTc2d 130400.9-774811) are partially contaminated by the flux of neighbor sources, most likely mimicking a flat spectrum. Therefore, none of these four sources can be considered as a YSO candidate. As seen below, this is further confirmed by applying new c2d selection criteria.

\subsection{New c2d Selection Criteria}

In order to improve the selection of the YSO candidates and clean the $\mathrm{c} 2 \mathrm{~d}$ samples as best as possible from extragalactic contamination, a new selection procedure was proposed by $\mathrm{H} 07$, using both IRAC and MIPS color and magnitude criteria, as well as a comparison with the resampled SWIRE catalogs matching the c2d sensitivity limits. To be able to use these criteria, the sources must be well detected $(\mathrm{S} / \mathrm{N} \geq 3)$ in all four IRAC bands and in MIPS $24 \mu \mathrm{m}$. This restricts the identification of the YSO candidates in the overlapping area surveyed in all four IRAC bands and MIPS $24 \mu \mathrm{m}$. A detailed description of the selection procedure can be found in the $\mathrm{c} 2 \mathrm{~d}$ final delivery document (Evans et al. 2007).

As a result of the multicolor procedure, applied to the bandfilled catalog in Cha II, 30 YSO candidates were selected in the IRAC $3.6-8 \mu \mathrm{m} /$ MIPS $24 \mu \mathrm{m}$ overlap area of $1.038 \mathrm{deg}^{2} .{ }^{13} \mathrm{Fig}-$ ure 3 shows the multicolor selection for Cha II; that figure is the equivalent to Figure 3 by H07. Five of the 30 objects are newly selected, while 25 coincide with already known certified or candidate YSOs reported in previous surveys (Young et al. 2005; Allers 2006; S07a and references therein). However, the latter reduce to 24 because 2MASS $12560549-7654106$, originally proposed as a YSO candidate by Young et al. (2005), has been spectroscopically confirmed to be a field star, unrelated to Cha II (see $\mathrm{S} 07 \mathrm{~b})$.

The recovery of all the 24 previously known objects in the IRAC 3.6-8 $\mu \mathrm{m}$ /MIPS $24 \mu \mathrm{m}$ overlapping area shows that the

\footnotetext{
13 The actual number of selected objects is 29 , but we stress that the wellknown young star IRAS $12496-7650$ (DK Cha) is saturated in IRAC 3.6 and $4.5 \mu \mathrm{m}$. By interpolating its SED in these bands, the object is definitely selected with the new c2d criteria. In fact, its $L$ and $M$ magnitudes from the literature (Hughes \& Hartigan 1992) are typical of a YSO.
}

new criteria work quite well in selecting sources with IR excess. More important is the fact that, except for the previously known Class I and flat-spectrum sources, all the other objects classified as Class I or flat-spectrum sources in Porras et al. (2007) are rejected, further supporting their extragalactic nature. Moreover, the previously confirmed Class I or flat-spectrum sources selected with the new criteria possess the highest luminosities in their Lada class (see Fig. 1). We thus conclude that the low-luminosity YSO candidates, selected using the previous criteria applied to IRAC data alone and classified as Class I or flat-spectrum sources in Porras et al. (2007), are indeed extragalactic contaminants.

A further test for the reliability of the new selection criteria can be performed by using the information available in the optical catalogs. An output parameter resulting from the source extraction with the SExtractor tool (Bertin \& Arnouts 1996) is the CLASS_STAR index. Pointlike objects have CLASS_STAR index very close to 1 , while for extended sources the index is very close to 0. Although some YSOs might appear as extended in some bands and some galaxies as pointlike, statistically one expects that most YSOs will show up as pointlike and galaxies as extended. In Figure 4 we show the histograms of the CLASS STAR index resulting from the $R$ and $I$ imaging for the objects classified as YSO candidates and galaxies after applying the new selection criteria. For the histograms we used the optical catalogs by S07a. The samples are well separated: sources classified as galaxies with the new multicolor criteria normally have CLASS STAR index close to 0 , while the index is typically 1 for the YSO candidates. Some galaxies may have CLASS_STAR index close to 1 , but the index is 1 for the vast majority of the YSO candidates. We thus conclude that the new selection criteria provide results that are consistent with the SExtractor morphological classification, giving further support for the new selection criteria to work well in cleaning the sample of YSO candidates from background extragalactic contaminants. Note that, while the morphological classification does separate extended from pointlike objects, some extended YSOs would escape selection if we rely on such selection alone; the c2 $\mathrm{d}$ multicolor criteria would select these as YSO candidates.

We now briefly discuss the five newly selected c2d sources: SSTc2d J124858.6-764154 and SSTc2d J125701.6-764835 are galaxies; the former coincides with the well-known galaxy LEDA 239204, while the latter is clearly identified with an interacting galaxy pair in our $I$-band images. Therefore, these two are rejected as YSO candidates. The remaining three sources show up as follows: SSTc2d J130521.7-773810 is a pointlike source and 


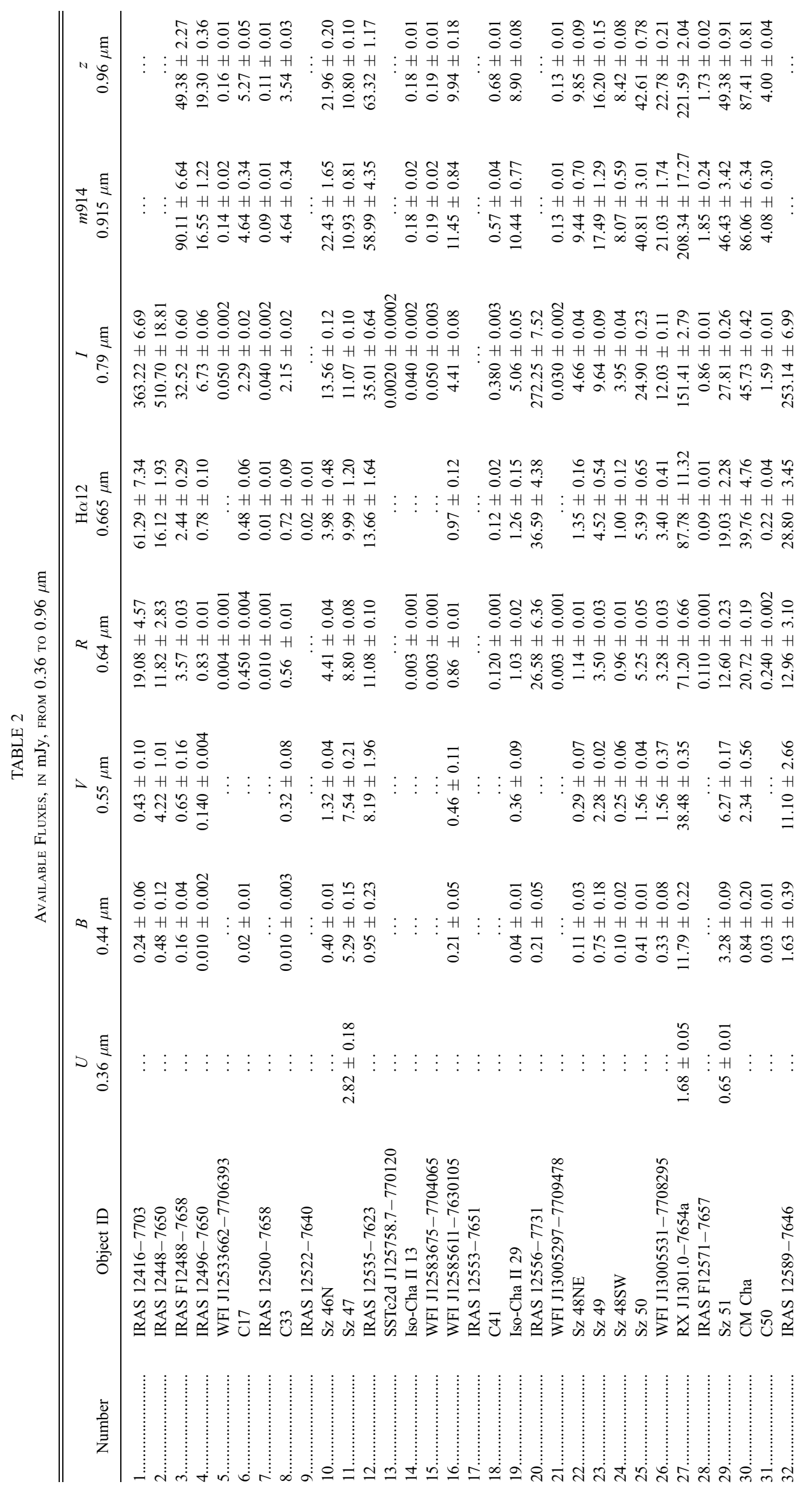




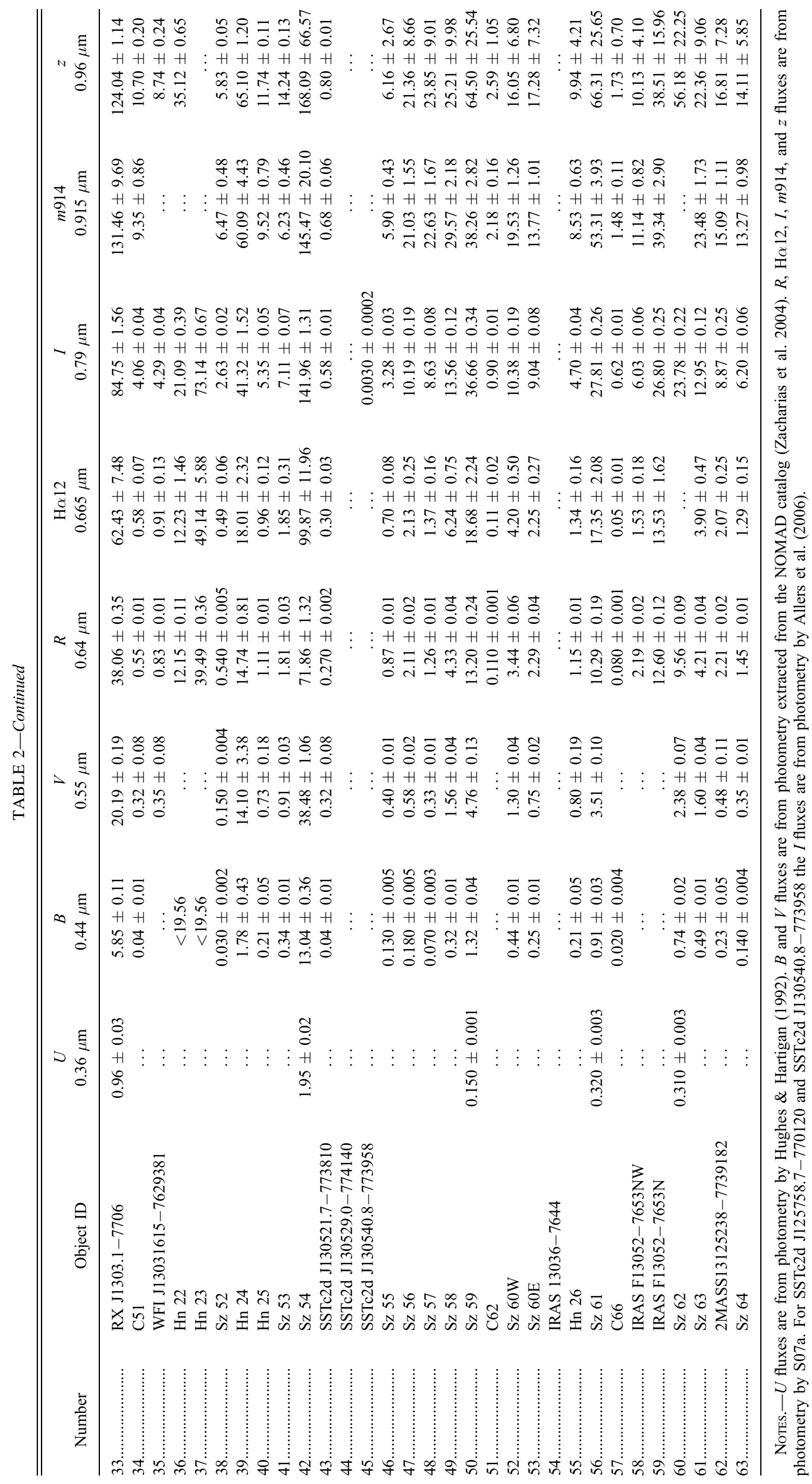




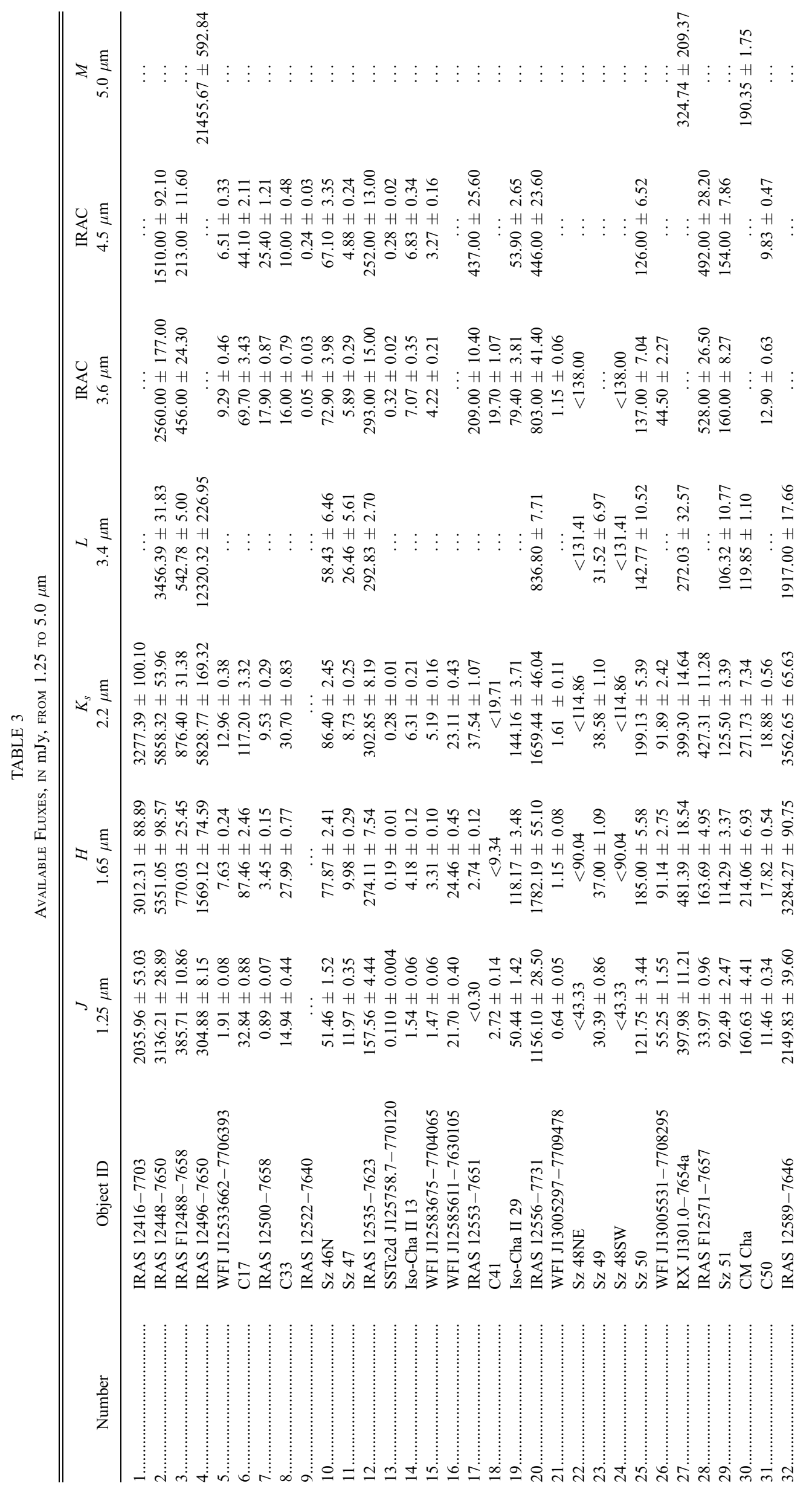




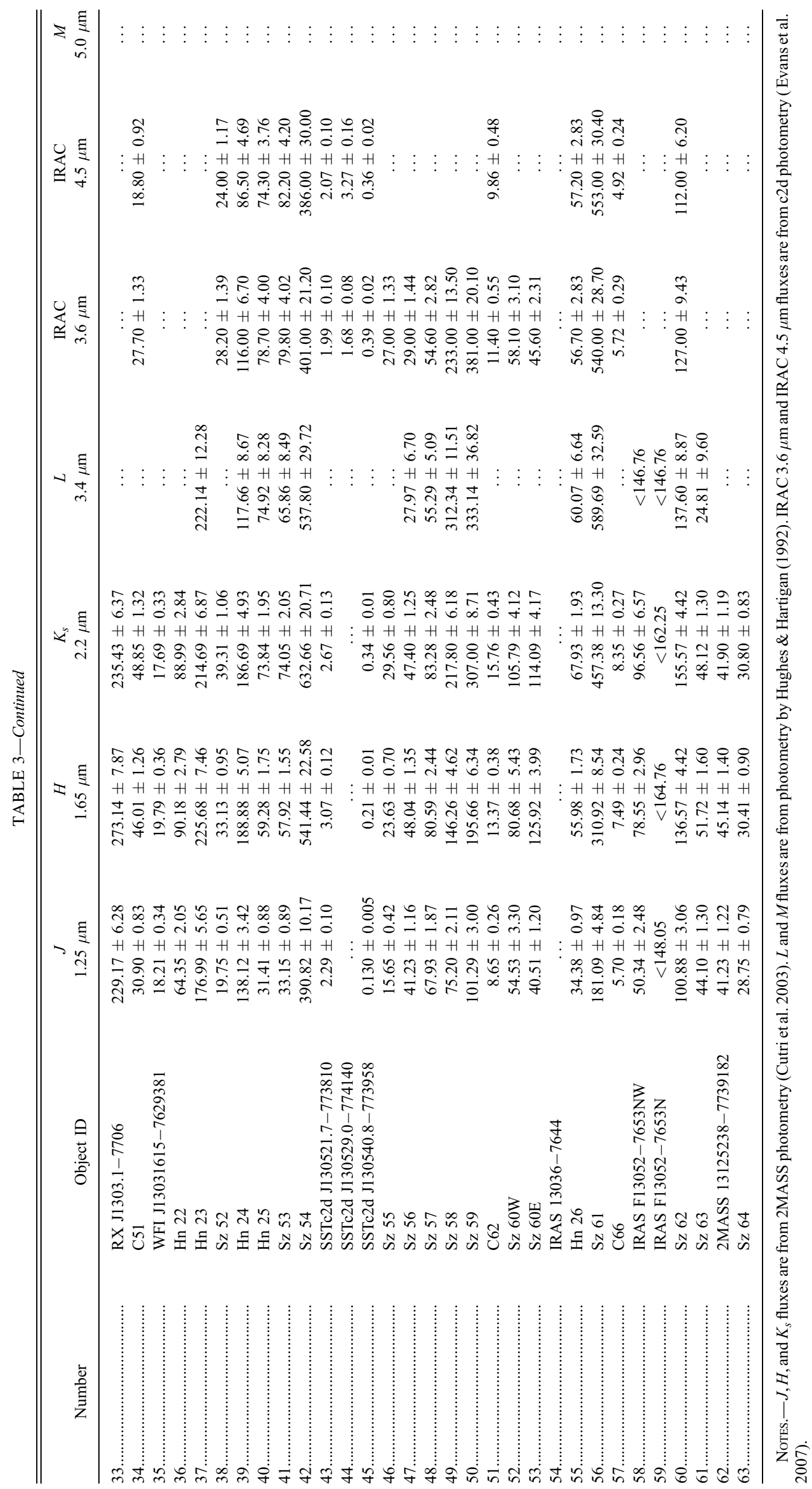




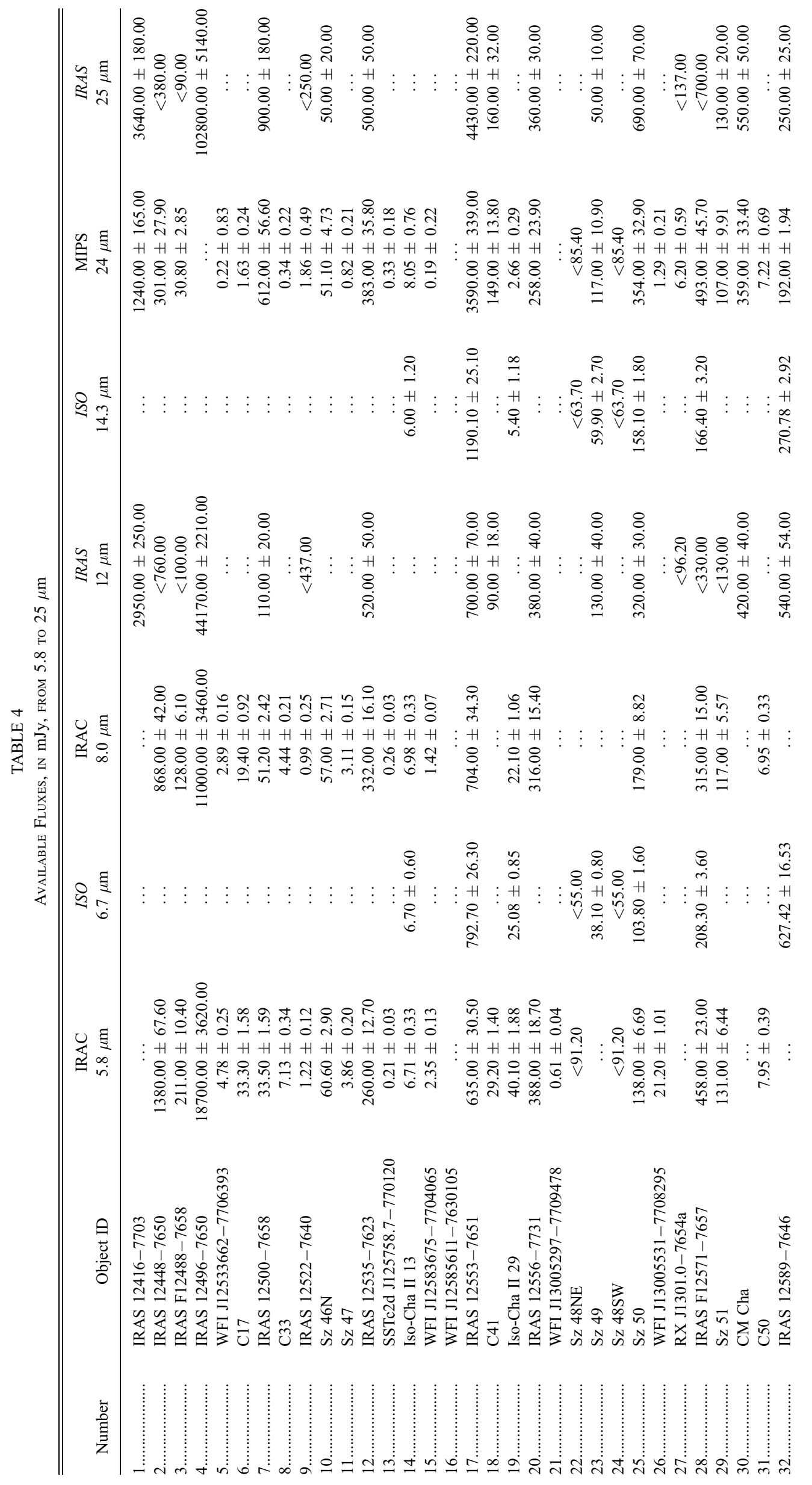




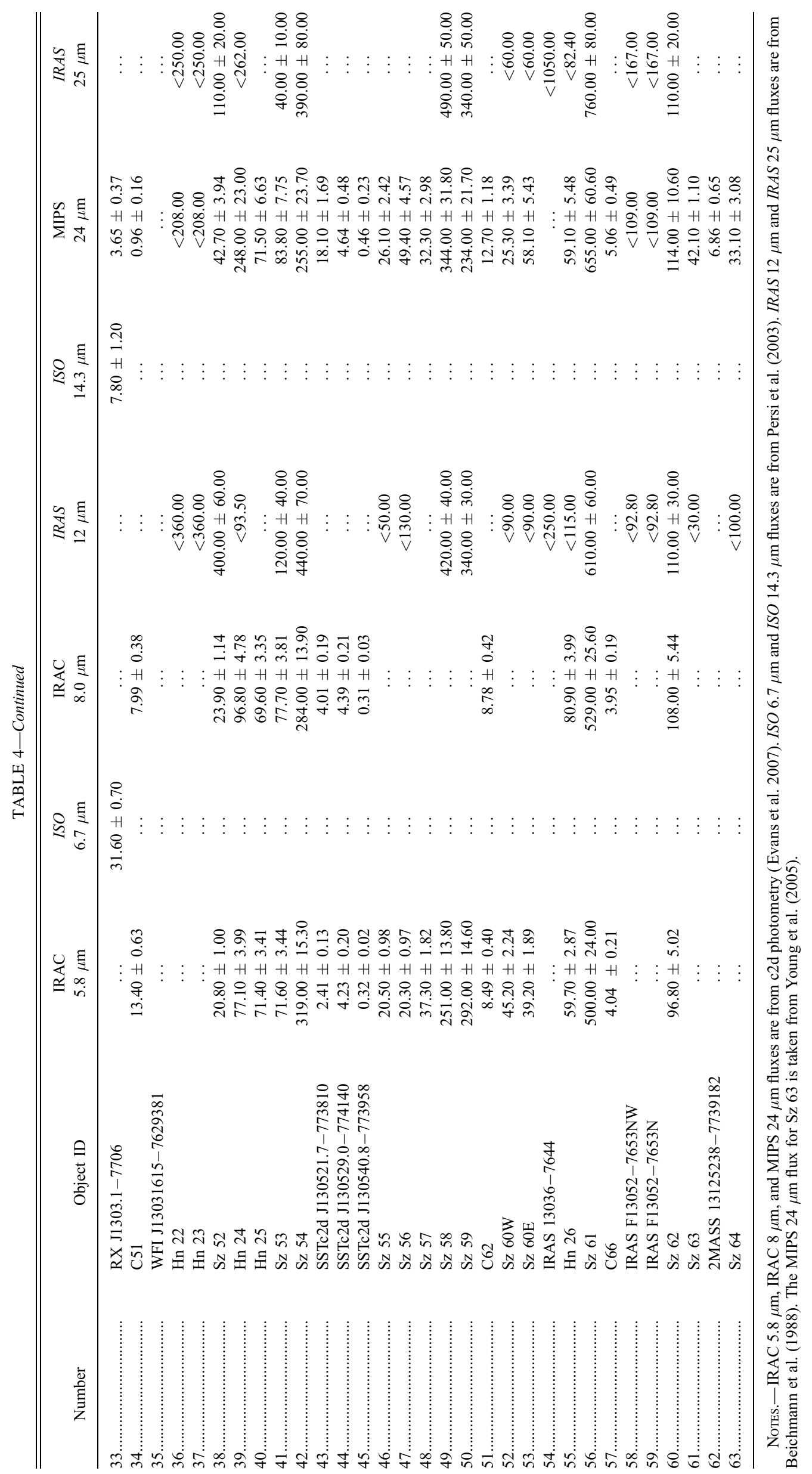




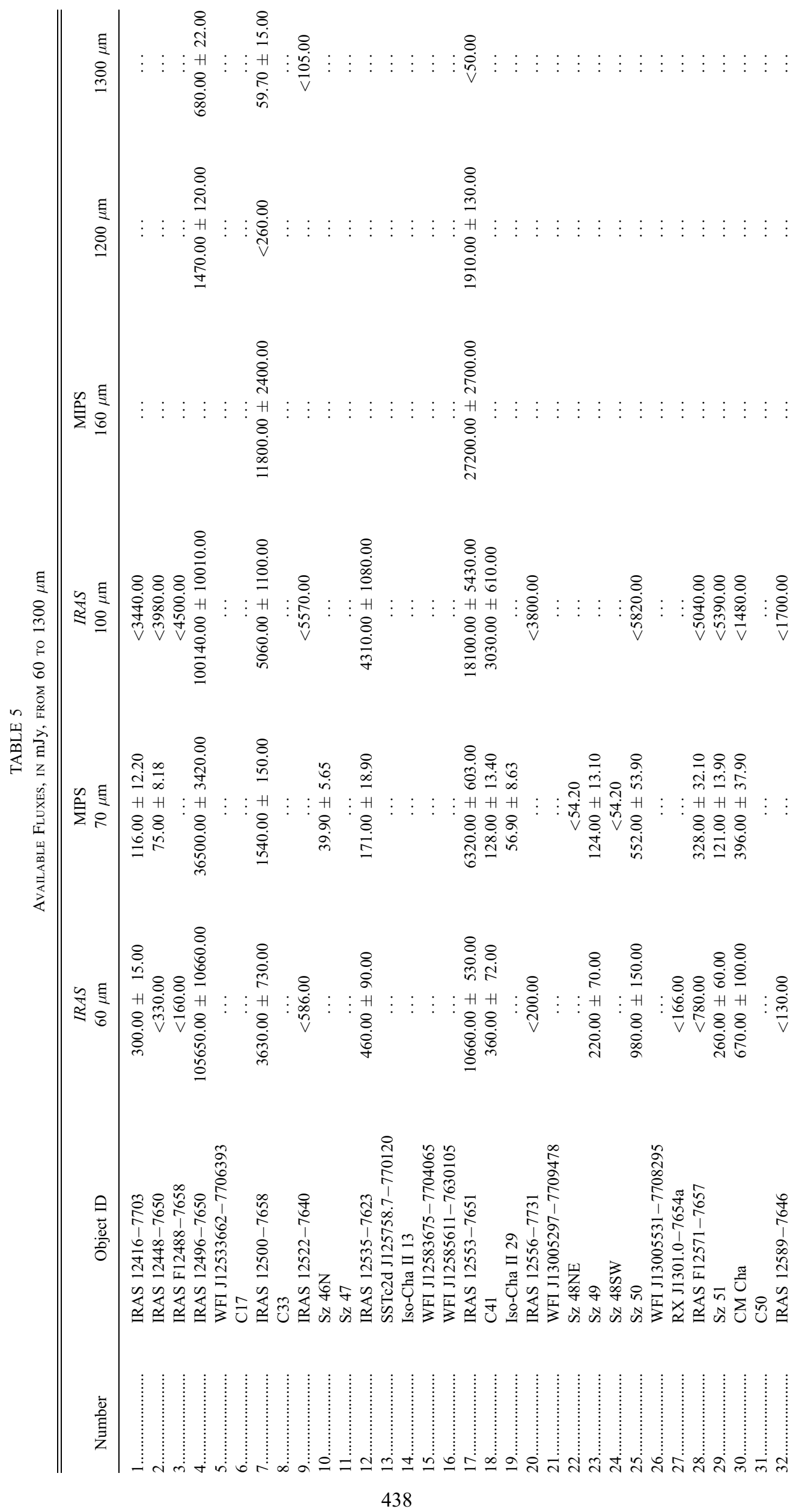




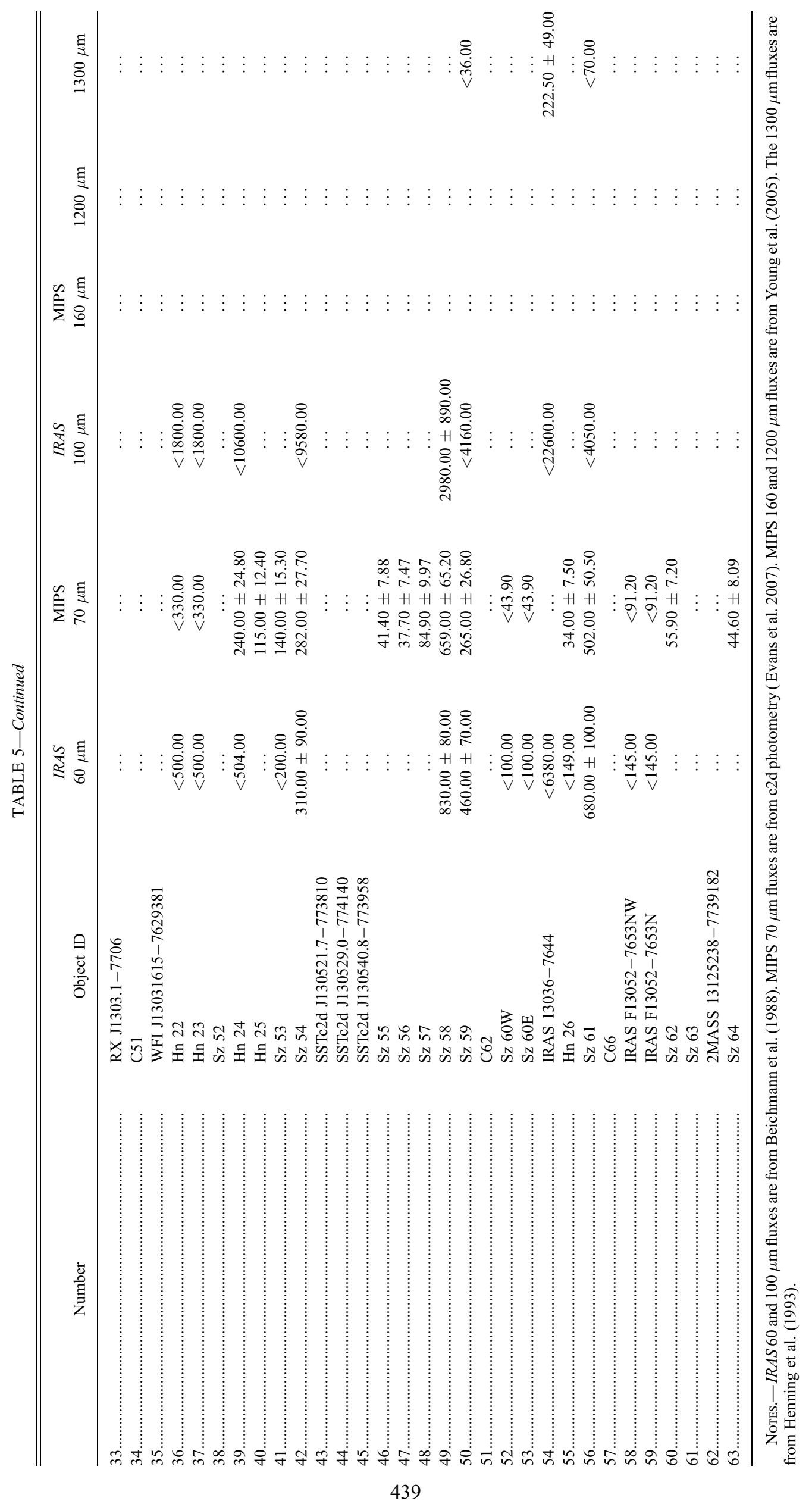




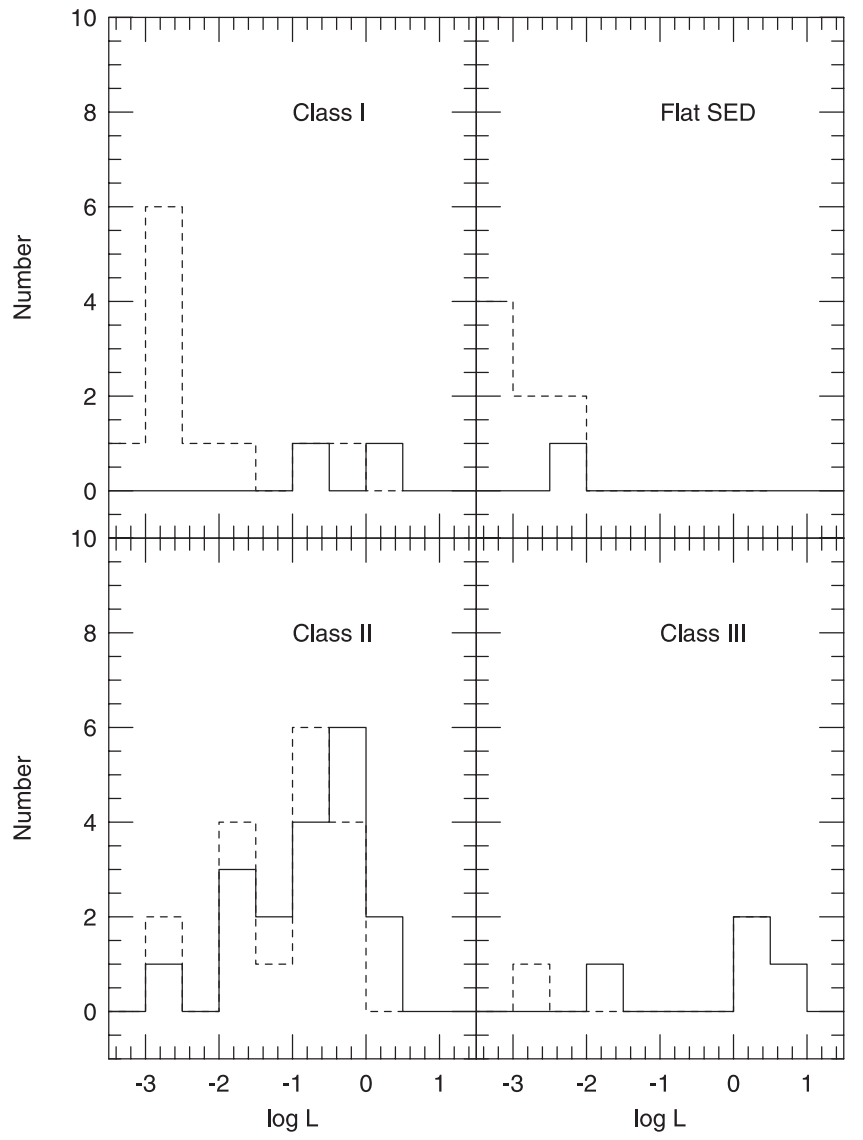

FIG. 1.-Luminosity histograms, in solar units, of the YSO candidates resulting from the selection criteria applied to the IRAC data alone (dashed histograms). Each panel represents a Lada class, as determined by Porras et al. (2007). Luminosities were derived by integration of the observed SEDs, assuming isotropic radiation and adopting the Cha II distance of $178 \mathrm{pc}$. The solid histograms represent the total sample of YSO candidates selected from the new criteria described in the text.

SSTc2d J130452.6-773947 is clearly a visual binary star in the $I$ band, while SSTc2d J130529.0-774140 was not detected in optical or NIR bands. SSTc2d J130521.7-773810 and both visual components of SSTc2d J130452.6-773947 are rejected as YSO candidates by the optical selection criteria by S07a. However, the former displays strong emission, as indicated by its photometric $\mathrm{H} \alpha$ index. Thus, its optical colors might be affected by strong veiling. Note that such YSOs may escape selection with the S07a criteria. Indeed, this object has been spectroscopically confirmed to show very strong $\mathrm{H} \alpha$ emission (S07b). On the other hand, SSTc2d J130452.6-773947 is strongly contaminated by the MIPS $24 \mu \mathrm{m}$ emission of Hn 24 (see $\S 3.4$ ), a certified YSO, explaining why its colors may apparently be consistent with those of YSOs. Considering that the higher resolution optical data reject this object and that no trace of $\mathrm{H} \alpha$ emission is found, we removed it from the list of YSO candidates. Finally, not being detected in optical and NIR bands, SSTc2d J130529.0-774140 cannot be ruled out as a YSO candidate. In conclusion, the three newly selected sources SSTc2d J124858.6-764154, SSTc2d J125701.6764835, and SSTc2d J130452.6-773947 are rejected as YSO candidates, while SSTc2d J130521.7-773810 and SSTc2d J130529.0-774140 can be considered as YSO candidates.

To summarize, in addition to the 24 previously known YSOs and candidates in the IRAC 3.6-8 $\mu \mathrm{m} /$ MIPS $24 \mu$ m overlapping area, there are at most two new potential YSOs. The members of the final list of 26 certified and candidate YSOs selected with the

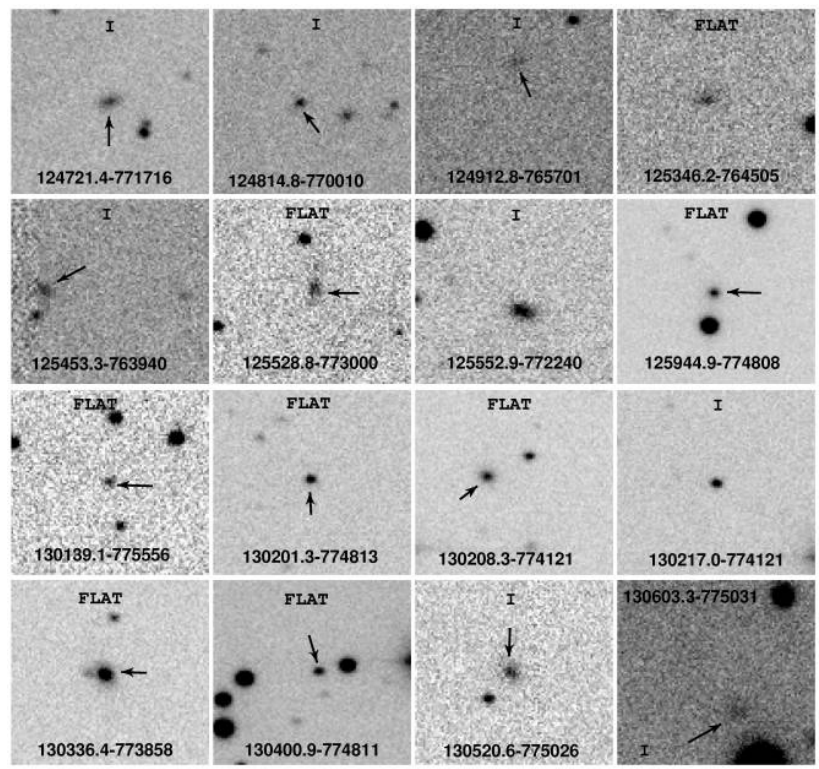

FIG. 2.- I-band images of objects classified as Class I or flat-spectrum sources by Porras et al. (2007). Each stamp covers an area of about $21 \times 21 \operatorname{arcsec}^{2}$. North is up and east to the left. The name of the sources and their classification are indicated. In case of ambiguity, the source is indicated with an arrow. Two sources (SSTc2d J125843.7-774728 and SSTc2d 125906.5-770740) are not shown because nothing is seen in the $I$ band, nor in any other optical image.

new multicolor criteria by $\mathrm{H} 07$ are marked with a flag ("YSO" or "YSO CND") in Table 1.

The selection criteria applied to the comparison resampled SWIRE data lead to the conclusion that $2 \pm 1$ of the selected sources in Cha II may be galaxies (see Fig. 3). Indeed, two of the five newly selected sources in Cha II, namely, SSTc2d J124858.6764154 and SSTc2d J125701.6-764835, were identified with galaxies. This result is similar to what $\mathrm{H} 07$ found for the Serpens molecular cloud.

\subsection{PMS Objects off the IRAC Area}

The areas mapped by the MIPS, optical, and NIR observations by Young et al. (2005), S07a, and Allers et al. (2006), respectively, are larger than the one covered with IRAC. The different selection criteria by these three surveys, besides providing new candidate PMS objects, coherently recovered the previously known certified and candidate PMS objects. Moreover, since the multicolor criteria select sources with IR excess emission, PMS objects not possessing substantial IR excess, at least somewhere in the range between 3.6 and $70 \mu \mathrm{m}$, escape selection. Thus, in Table 1 we include also the PMS objects found in the aforementioned MIPS, optical, and NIR surveys. As reported in $\S 3$, the complete sample includes 62 certified and candidate PMS objects.

The luminosity function of the selected YSO candidates (see $\S 5.5)$ demonstrates that the $\mathrm{c} 2 \mathrm{~d}$ observations are basically complete down to $\log \left(L / L_{\odot}\right) \approx-2.5$ in the IRAC $3.6-8 \mu \mathrm{m} /$ MIPS $24 \mu \mathrm{m}$ overlap area. ${ }^{14}$ In the case of Serpens, H07 extrapolate the number of YSO candidates selected in the IRAC 3.6-8 $\mu \mathrm{m} / \mathrm{MIPS} 24 \mu \mathrm{m}$ overlap area to the larger area surveyed only with MIPS using the percentages of sources classified as YSOs from different criteria. Following a similar reasoning, we can investigate the expected number of YSOs if IRAC observations had been performed in the entire area covered by MIPS $24 \mu \mathrm{m}$ in Cha II. Young et al.

\footnotetext{
14 This luminosity limit corresponds to a mass $M \approx 0.03 M_{\odot}$, assuming the Chabrier et al. (2000) PMS evolutionary tracks for objects younger than $10 \mathrm{Myr}$.
} 

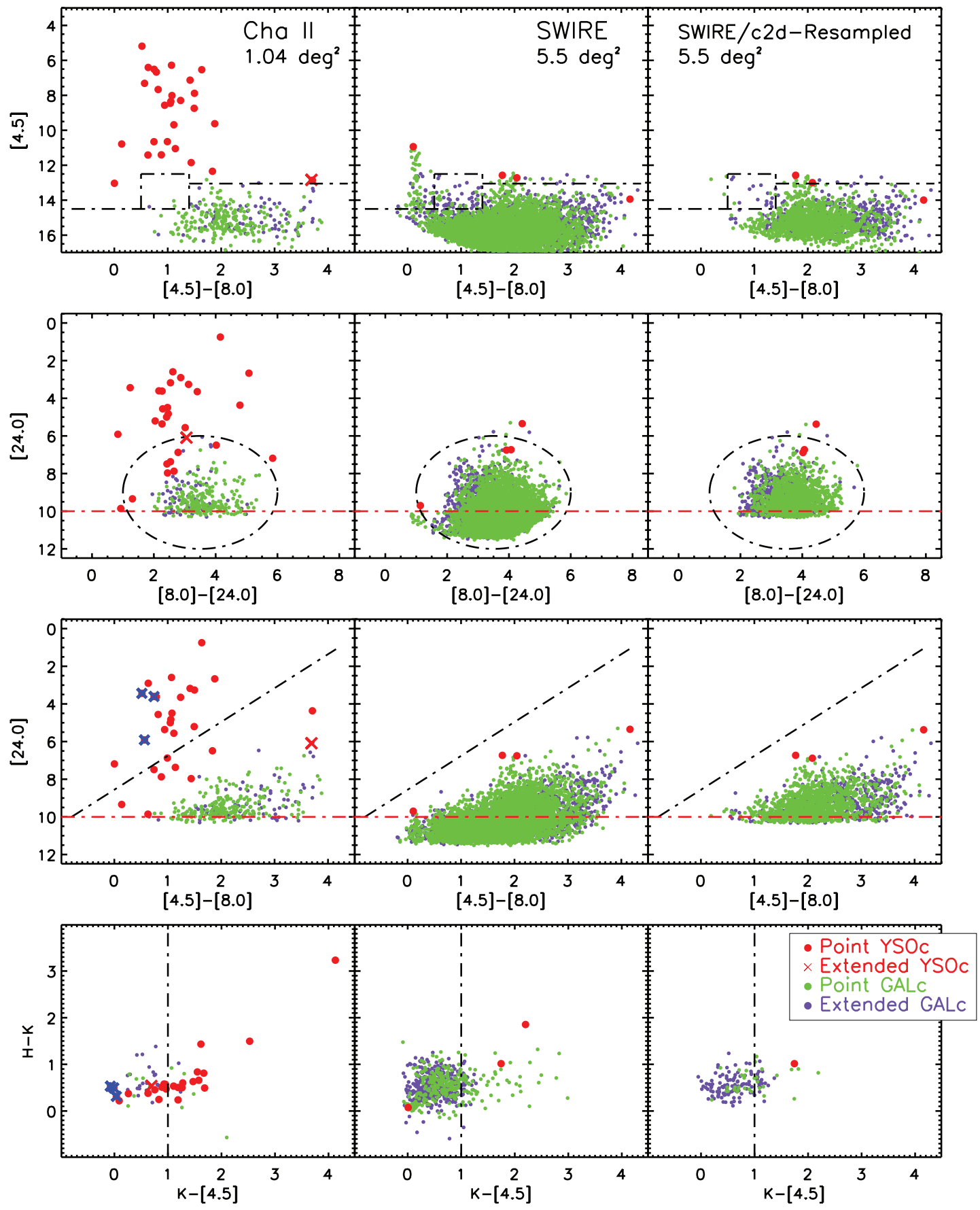

Fig. 3.-Color-magnitude and color-color diagrams for the Cha II cloud (left), the full SWIRE catalog (middle), and the resampled SWIRE catalog (right). The dotdashed lines represent the color-magnitude cuts defining the YSO candidate criteria according to $\mathrm{H} 07$.

(2005) selected 44 YSOs based on the combination of MIPS and 2MASS data; 20 of these fall outside the area observed with IRAC. ${ }^{15}$ Of the 24 objects selected by Young et al. (2005) in the IRAC 3.6-8 $\mu \mathrm{m}$ /MIPS $24 \mu \mathrm{m}$ overlap area in Cha II, 23 satisfy the $\mathrm{H} 07$ multicolor criteria presented in this work, i.e., $96 \%$. This means that $4 \%$ of the 20 objects $(\sim 1)$ outside the IRAC $3.6-8 \mu \mathrm{m} /$ MIPS $24 \mu \mathrm{m}$ overlap area would have been rejected by the new multicolor criteria. Now, 24 of the 26 YSOs selected in the IRAC 3.6-8 $\mu \mathrm{m} / \mathrm{MIPS} 24 \mu \mathrm{m}$ overlap area in this work are recovered by the Young et al. (2005) criteria, which means that

\footnotetext{
15 We stress, however, that 3 objects (Sz 48, Sz 60, and IRAS F13052-7653) out of these 20 are multiple visual systems that are blended in the MIPS data by Young et al. (2005)
}

about $8 \%$ of the 20 objects $(1-2)$ outside the IRAC $3.6-8 \mu \mathrm{m} /$ MIPS $24 \mu \mathrm{m}$ overlap are missed by the Young et al. (2005) criteria. All this leads to an expected number of about 21 YSOs in the area observed only with MIPS $24 \mu \mathrm{m}$. Therefore, the expected number of YSOs if IRAC observations had been performed in the entire area covered by MIPS $24 \mu \mathrm{m}$ would be something like $26+21=47$. This means that about $24 \%$ of the 62 certified and candidate PMS objects mentioned above should not display significant IR excess in any of the Spitzer bands and thus are expected to be preferentially Class III sources. This conclusion is in agreement with the results of our census (see $\S \S 3.1$ and 5.3). Therefore, besides having a complete sample also outside the IRAC $3.6-8 \mu \mathrm{m} /$ MIPS $24 \mu \mathrm{m}$ overlap area, and despite the low number statistics, our results provide support to the arguments by 

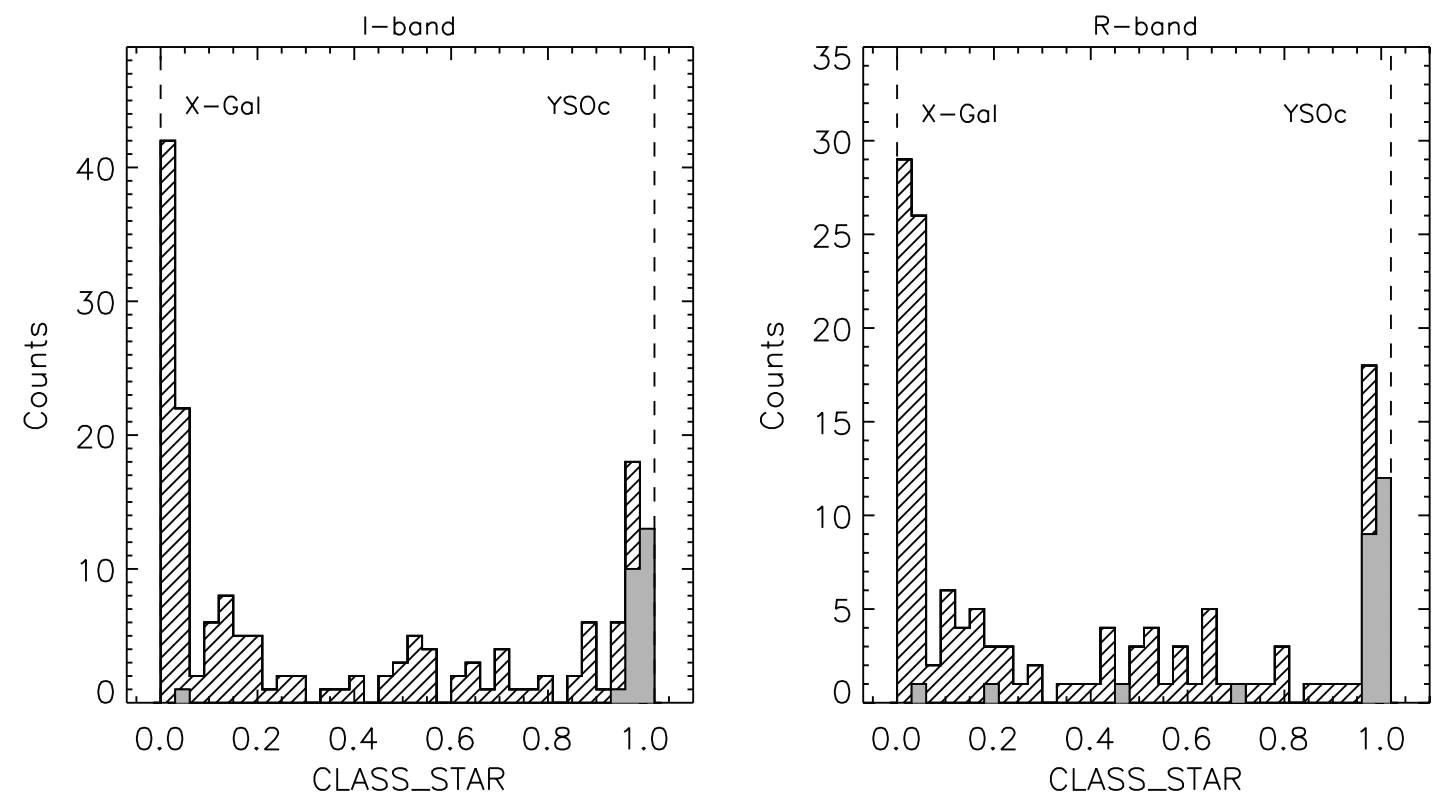

FIG. 4.-Histograms of SExtractor CLASS_STAR indices of the sources extracted from the $R$ - $(r i g h t)$ and $I$-band (left) images by S07a. The hatched histograms correspond to sources having high probability of being extended from the new multicolor criteria, while the gray ones correspond to the pointlike sources. The dashed lines at CLASS_STAR 0 and 1 represent the limits for extended and pointlike objects, respectively. The only YSO candidate with CLASS_STAR index close to 0 in both bands corresponds to the interacting galaxies discussed in the text.

H07 for the extrapolation of the number of YSOs to the areas observed only with MIPS.

\subsection{Multiple Visual Systems}

In order to avoid spurious selections due to crowding, we have visually inspected all the confirmed and candidate PMS objects in all available images, from optical to MIPS $24 \mu \mathrm{m}$. The result is that seven objects are found to have neighbors within less than $10^{\prime \prime}$ in our optical images and may be blended in the IRAC or MIPS images. In some cases they appear as single sources, in particular in the MIPS images, where the spatial resolution is worse $\left(\sim 6^{\prime \prime}\right.$ at $24 \mu \mathrm{m}$ and $\sim 17^{\prime \prime}$ at $\left.70 \mu \mathrm{m}\right)$ with respect to IRAC images $\left(<2^{\prime \prime}\right)$. We now discuss these objects individually.

SSTc2d J130452.6-773947.- - In the $R$-band images this source coincides with a visual binary system with a separation of $1.6^{\prime \prime}$. The PMS star Hn 24 is located at about 10" to the east of it. Hn 24 itself is a visual binary with a separation of about $1.8^{\prime \prime}$. In the MIPS $24 \mu \mathrm{m}$ image Hn 24 and SSTc2d J130452.6-773947 appear as a single object; hence, the MIPS $24 \mu \mathrm{m}$ emission of SSTc2d J130452.6-773947 is strongly contaminated by the $24 \mu \mathrm{m}$ emission from $\mathrm{Hn} 24$. The quality flags from the new band-filled c2d catalog indicate that, although SSTc2d J130452.6773947 was well detected in the four IRAC bands, only an upper flux limit could be determined in MIPS $24 \mu \mathrm{m}$. Therefore, it is most likely that Hn 24 is the main source of the $24 \mu \mathrm{m}$ emission. In fact, the optical magnitudes and colors of both components of SSTc2d J130452.6-773947 do not satisfy the selection criteria by S07a, nor do their $\mathrm{H} \alpha$ photometric indices indicate emission. Therefore, SSTc2d J130452.6-773947 is most likely a spurious selection.

IRAS F13052-7653.- - In the optical this object is revealed as a triple visual system (see S07a). The north and southwest components are the brightest, while the northwest component is the faintest one. The separation of the bright components is about $3^{\prime \prime}$, while the separation of IRAS F13052-7653N and IRAS F13052$7653 \mathrm{NW}$ is about $4^{\prime \prime}$. The latter two, while being potential candidate PMS objects based also on optical criteria by S07a, have been spectroscopically confirmed to be PMS stars (S07b). The op- tical magnitudes and colors of the south component are inconsistent with those of Cha II PMS stars (S07a). The visual triple was not observed with IRAC, but in MIPS $24 \mu \mathrm{m}$ the three visual components are blended in a single source.

Hn 22 and Hn 23.--In optical images these PMS stars appear as a visual pair with a separation of about $6^{\prime \prime}$ in the northeastsouthwest direction. In the $R$ band the northeast component, $\mathrm{Hn} 23$, is brighter than the southwest component $\mathrm{Hn} 22$. The visual pair was not observed with IRAC, but an elongated structure in the MIPS $24 \mu \mathrm{m}$ images can be appreciated.

$S z$ 48. - This object appears as a visual pair with a separation of about $1.5^{\prime \prime}$ in the northeast-southwest direction in the $R$-band image. The visual binary appears as a single object in the Spitzer data. The new band-filled c $2 \mathrm{~d}$ catalog lists a single detection in IRAC $3.6 \mu \mathrm{m}$, IRAC $5.8 \mu \mathrm{m}$, MIPS $24 \mu \mathrm{m}$, and MIPS $70 \mu \mathrm{m}$. In the MIPS $70 \mu \mathrm{m}$ image the object falls quite near the border. This pair was also studied by Brandner \& Zinnecker (1997).

Sz 59. - This object has been detected by Brandner \& Zinnecker (1997) to be a visual binary with a separation of $0.78^{\prime \prime}$. The binary appears as a single object in optical and IR images.

$S z 60$.- This is a visual pair with a separation of about $3^{\prime \prime}$ in the east-west direction in optical images. Both components were detected in IRAC $3.6 \mu \mathrm{m}$, IRAC $5.8 \mu \mathrm{m}$, and MIPS $24 \mu \mathrm{m}$, but their $\mathrm{S} / \mathrm{N}$ is too low to perform shape analysis. The west component was well detected in MIPS $70 \mu \mathrm{m}$, but only an upper limit in this band could be determined for the east component.

Sz 62.-Brandner \& Zinnecker (1997) report this object as a binary with a separation of $1.1^{\prime \prime}$. The object also appears as a visual binary in our optical images and shows up as a single object in all IR images.

Because of the difficulty to deblend the components of these objects in the Spitzer images, we consider their c2d fluxes as upper limits in Tables 2, 3, 4, and 5. The range of separation of the certified and candidate PMS objects is from about $0.8^{\prime \prime}$ to $6^{\prime \prime}$, the former being about the limiting separation we can resolve with the WFI images, mainly due to the seeing (see S07a). This range of separations means about 140-1060 AU at the distance of Cha II. Considering that among the above objects there are six legitimate 

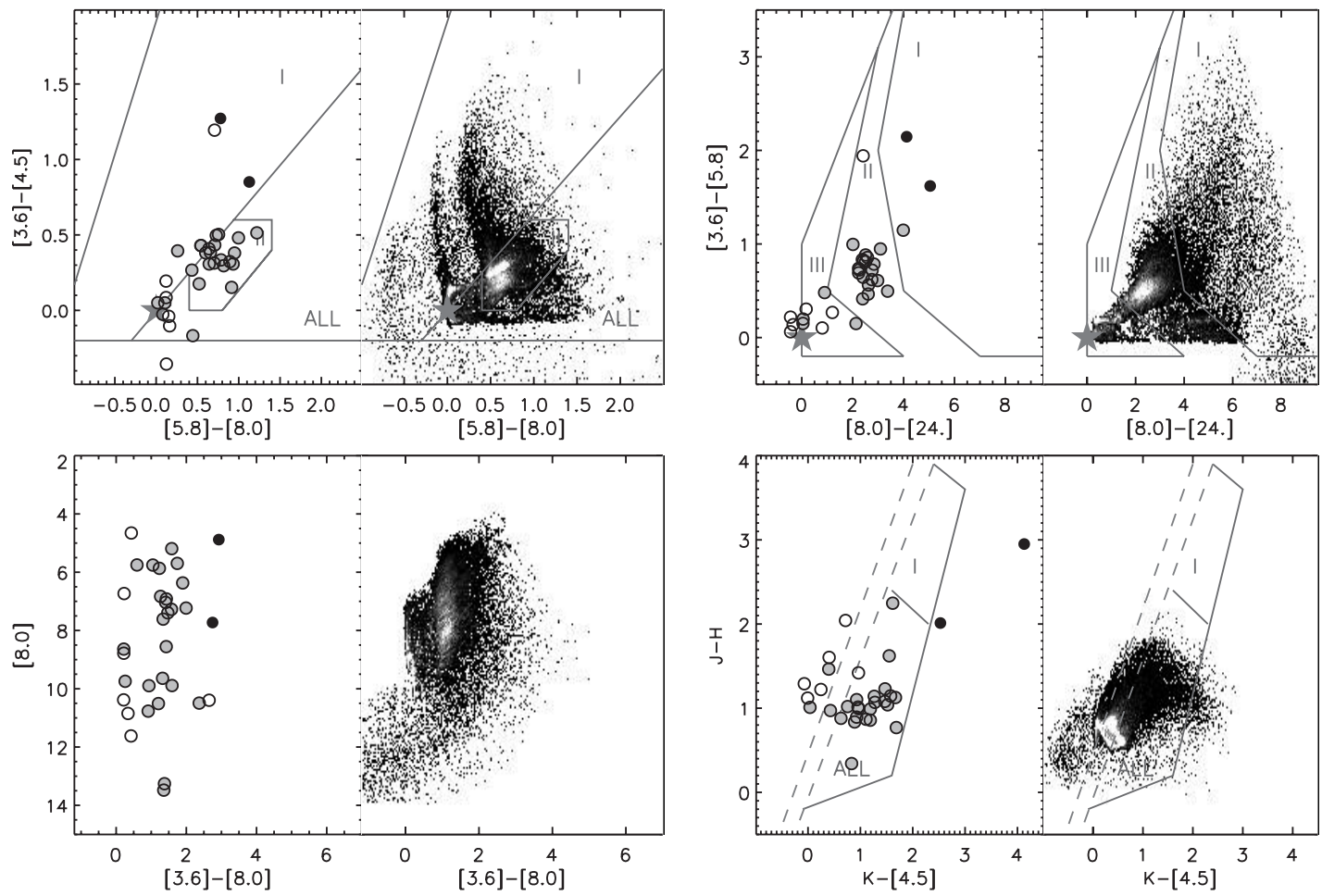

FIG. 5.-Color-color and color-magnitude diagrams for the spectroscopically confirmed ( gray filled circles) and candidate (open circles) PMS objects with Spitzer and 2MASS data are plotted on the left side of each panel. The objects classified as Class I sources are represented with black filled circles. The colors derived from the SED models by R06 are plotted (in gray-scale intensity representing the density of points) on the right side of each panel. The areas corresponding to Stages I, II, and III as defined by R06 are also indicated in each diagram. The label "ALL" marks the regions where models of all evolutionary stages can be present. The big star represents normal unreddened photospheres. The majority of PMS stars in Cha II fall in regions corresponding to Stage II sources. [See the electronic edition of the Journal for a color version of this figure.]

PMS objects and taking into account uncertainties on the final number of true cloud members, we find a multiplicity fraction of the order of $13 \% \pm 3 \%$, which is consistent with the observed fraction in PMS clusters in the same range of separations (see Padgett et al. 1997). The result is also consistent with the binary fraction of $14.0 \% \pm 4.3 \%$ reported by Köhler (2001) for stars in the Chamaeleon complex, with separations of $0.13^{\prime \prime}-6^{\prime \prime}$. We do not see any dependence of binarity on spectral type, but the statistics are poor.

\section{SEARCH FOR VARIABILITY IN THE SPITZER BANDS}

Since the Spitzer data were gathered in two epochs separated by about $6 \mathrm{hr}$, we can search for variability on that timescale, similarly as has been done in other c2d papers (H07; Rebull et al. 2007). In order to be able to assess variability, the investigated sources need first to be detected with a good confidence level in both epochs, and the artifacts due to cosmic rays, detections in image borders, etc., need to be cleaned up. Thus, in each band, we have selected all the sources detected in both epochs with an $\mathrm{S} / \mathrm{N}$ greater than or equal to 3 and checked for cosmetics in connection with objects close to the image border or bright stars.

We did not find significant variability on the timescale of $6 \mathrm{hr}$ for the Cha II sources in any of the IRAC bands and in MIPS $24 \mu \mathrm{m}$. This implies that none of the PMS objects or candidates show variability in the Spitzer bands on that timescale. This is in line with the $\mathrm{c} 2 \mathrm{~d}$ findings in other star-forming regions like Serpens (H07) and Perseus (Rebull et al. 2007).

\section{CIRCUMSTELLAR DUST PROPERTIES IN CHA II}

\subsection{Color-Color and Color-Magnitude Diagrams}

Infrared color-color (CC) and color-magnitude (CM) diagrams are good diagnostic tools for the investigation of circumstellar matter around YSOs (Hartmann et al. 2005; Lada et al. 2006 and references therein). The traditional Lada (1987) classification of classes based on the slope of the SED alone may sometimes lead to misclassification. For instance, an embedded Class I source seen pole-on may display a flat spectrum, while a normal disk seen edge-on can be confused with a Class I object. In order to avoid confusion between the Lada classes and their evolutionary status, Robitaille et al. (2006, hereafter R06) introduced the term "stages," which refers to the true evolutionary stage of the sources as follows: Stages 0-I have significant envelopes and possibly disks, Stage II objects have optically thick disks, while sources with optically thin disks are classified as Stage III sources.

About $60 \%$ of the sample reported in Table 1 have sufficient data to perform an analysis in CC and CM diagrams similar to the one presented in previous c2d papers (e.g., H07). Figure 5 shows CC and CM diagrams in the Spitzer and 2MASS bands. The diagrams are compared with those derived from the models by $\mathrm{R} 06$, adjusted in order to fit the distance and mass distribution of Cha II and matching the sensitivity cutoff for the 2MASS (Cutri et al. 2003) and Spitzer bands (Harvey et al. 2006; Jørgensen et al. 2006). Although the number of objects in Cha II for which we can perform this analysis is small, the ranges of colors and magnitudes are those expected for YSOs in different evolutionary stages. For instance, it is evident that only a handful of Stage I sources exist in Cha II. Only two YSOs have colors similar to Stage I sources; these are Iso-Cha II 28 and IRAS 12500-7658, which were classified as Class I IR sources in previous works (Young et al. 2005 and references therein). The comparison with the models indicates that the majority of the previously known PMS objects have colors consistent with those belonging to Stage II, while most of the candidate PMS objects have IR colors similar to those of Stage III; the exception is the new Spitzer YSO 

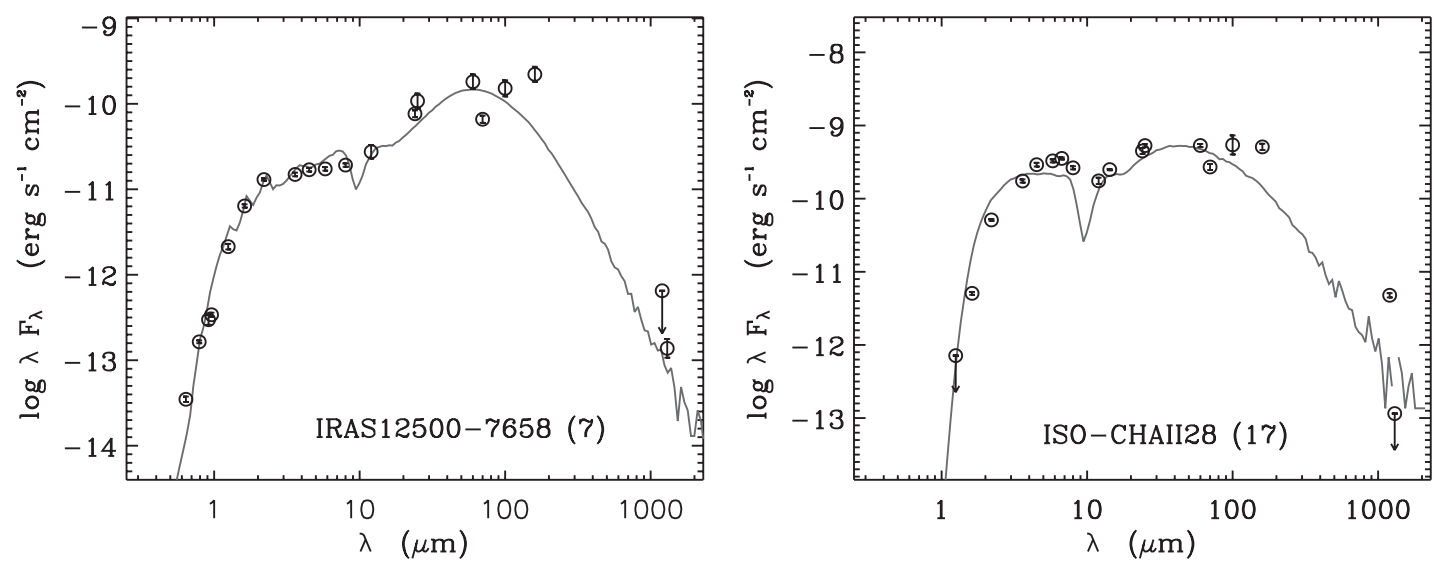

FIG. 6. - SEDs of the two Class I YSOs in Cha II. Complementary ancillary data in millimeter wavelengths for IRAS 12500-7658 are from Henning et al. (1993).

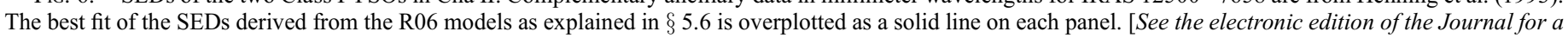
color version of this figure.]

candidate SSTc2d J130521.7-773810, which has colors consistent with Stage II objects.

Differently to what is found in $\mathrm{H} 07$ for Serpens, we do not have sources with very red colors in the [8.0] versus [3.8] - [8.0] diagram. The smaller percentage of very red objects might in prin- ciple be due to a more evolved evolutionary status of the YSO population; however, these evolved objects are not found in big numbers in Cha II. Thus, the lack of very red objects is probably a consequence of the low number statistics. On the other hand, while the number of Stage I sources is quite small relative to the
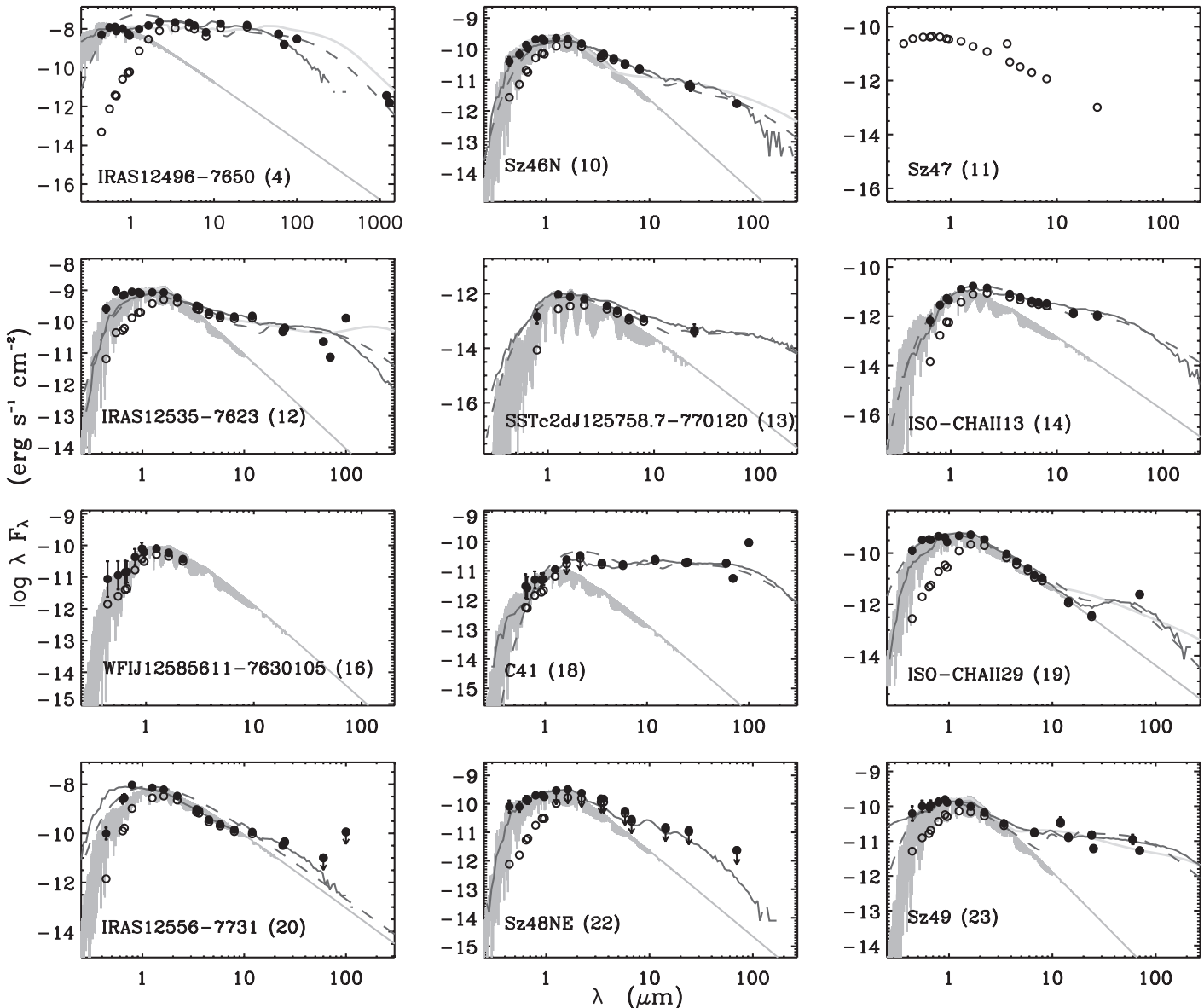

FIG. 7.-SEDs of the Class II and Class III PMS objects in Cha II. The dereddened fluxes are represented with filled circles, while the observed fluxes are represented with open circles. The best-fit NextGen spectrum (by Hauschildt et al. [1999] for objects with $T_{\text {eff }}>4000 \mathrm{~K}$ ) or StarDusty spectrum (by Allard et al. [2000] for objects with $T_{\text {eff }}<4000 \mathrm{~K}$ ) is overplotted on each SED, representing the stellar flux. The best fits of the SEDs derived from both the R06 and D01 models are overplotted as solid and dashed lines, respectively, while those derived from the D'Alessio et al. (2005) models are represented with the thick lines. Note that a reasonable fit to the stellar and dust emission of the veiled object Sz 47 could not be obtained, while for WFI J12585611-7630105 the dust emission could not be modeled because of insufficient IR photometry. The modeling was not attempted for those sources showing pure photospheric Class III SEDs (see $\S$ 5.6). Each panel is labeled with the object's name and its entry number from Table 1 in parentheses. [See the electronic edition of the Journal for a color version of this figure.] 

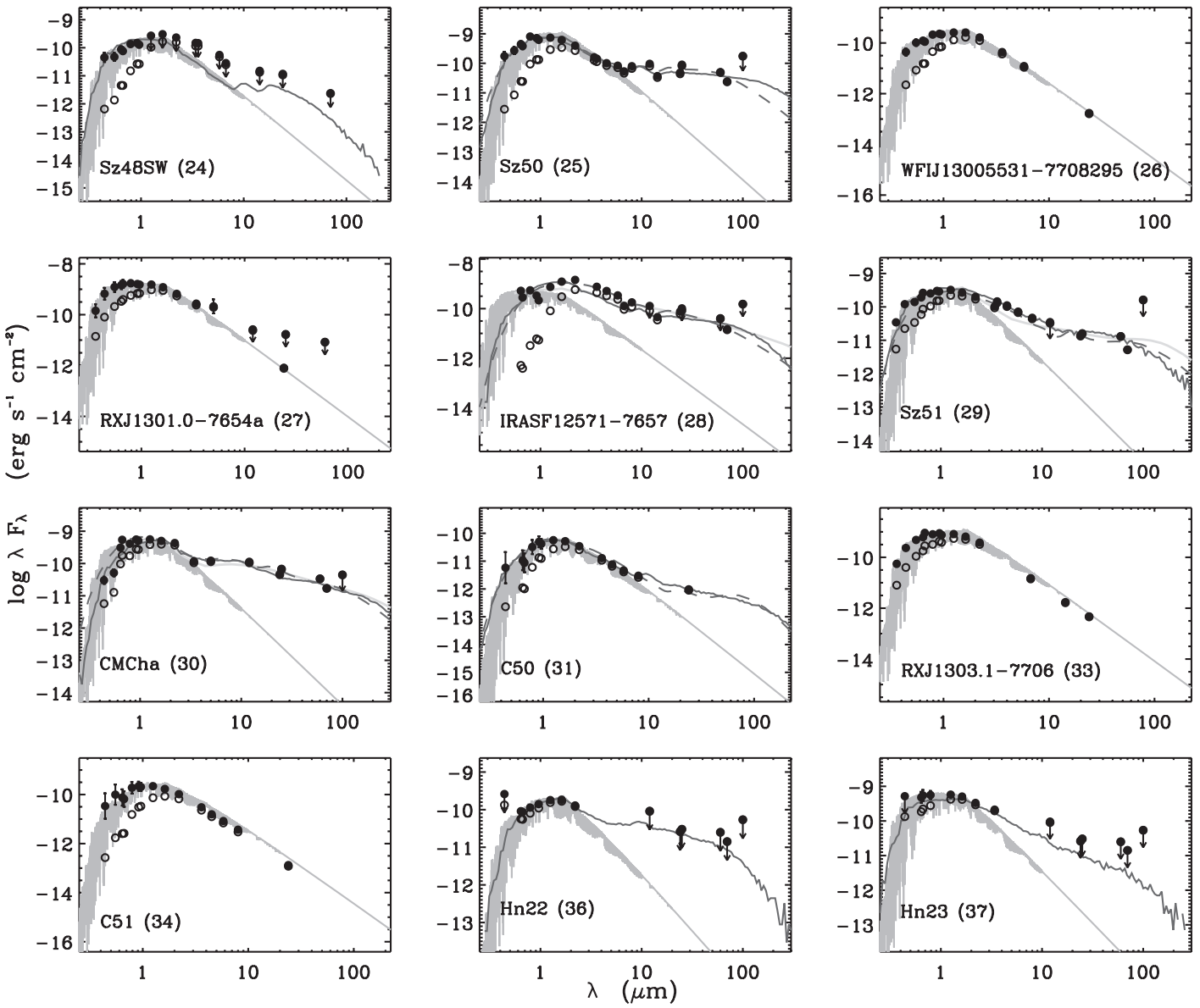

Fig. 7-Continued

number of Stage II sources, the disk fraction is quite high (see $\S 5.9)$. This may mean that either (1) star formation in Chamaeleon has occurred in a burst a few million years ago or (2) the disk evolution timescale may be different as in other regions like Serpens, Perseus, and Ophiuchus. The discussion on triggered star formation in Chamaeleon presented in $\S 5.4$ seems to be consistent with the former hypothesis.

The IR colors of the very low mass objects $\left(M<0.2 M_{\odot}\right)$ in Cha II are very similar to those of Class II sources. We use here the average mass for each individual object as determined by S07b. We note that the colors of these very low mass objects are also consistent with those of Stage II. This shows, as previous studies do (e.g., Allers et al. 2006), that disks are also common in very low mass objects, down to the substellar regime.

The analysis of the SEDs reported in $\S 5.2$ implies that the conclusions drawn from the $\mathrm{CC}$ and $\mathrm{CM}$ diagrams discussed above can be extrapolated to the whole sample of 62 objects. Therefore, we conclude that most objects in Cha II have IR colors similar to those of Class II sources, with only a few corresponding to Class I and Class III sources, and that most PMS objects in this cloud are in the evolutionary Stage II. A few Class III objects may be missed, however, by the multicolor criteria by H07 in the IRAC 3.6-8 $\mu \mathrm{m} /$ MIPS $24 \mu \mathrm{m}$ overlapping area. More discussion on the source IR classes is provided in the next sections.

\subsection{Spectral Energy Distributions}

In order to study in more detail the circumstellar matter around the certified and candidate PMS objects, we have determined their SEDs from the optical to millimeter wavelengths depending on available data. These SEDs, corrected for interstellar extinction, can be used to model the structure of the PMS circumstellar material. To this aim, the flux at each wavelength must be corrected for reddening. The extinction has been determined for each individual PMS object and candidate using the spectral types provided in $\mathrm{S} 07 \mathrm{~b}$ and the extinction law by Weingartner \& Draine (2001) for $R_{V}=5.5$ (hereafter WD5.5; see also $\S 6.2$ ). For the details we refer the reader to $\mathrm{S} 07 \mathrm{~b}$. The uncertainties on $A_{V}$, derived from the uncertainties in temperature, range from 0.1 to less than $1 \mathrm{mag}$. Our extinction determinations are in general agreement with the values reported by the large-scale extinction maps of the Cha II cloud by Cambrésy (1999), J. Kainulainen (2006, private communication), and the c2d extinction map (discussed in $\S 6.2$ ) within less than 2 mag for objects with $A_{V}$ lower than about 4 mag, while for those with higher values the maps tend to provide higher $A_{V}$ values. This might mean that the certified and candidate PMS objects are not behind the cloud or may be a consequence of the large beams used to make the extinction maps.

A different approach was followed for the objects lacking spectroscopy. In these cases, the $A_{V}$ and temperature values were derived following the prescription by S07a, but using the WD5.5 extinction law. These methods yield consistent temperatures with those determined from spectroscopy, with residuals less than $200 \mathrm{~K}$ provided that the extinction is not high, which is the case for most of the candidate PMS objects in Cha II. Moreover, since most of these candidates are expected to be Class III sources (see $\S \S 5.1$ and 5.3), those methods to estimate effective temperature can be applied confidently.

In the case of the embedded Class I sources IRAS 125007658 and Iso-Cha II 28 , for which we do not know the temperature, we cannot apply the above procedures. Since most of the 

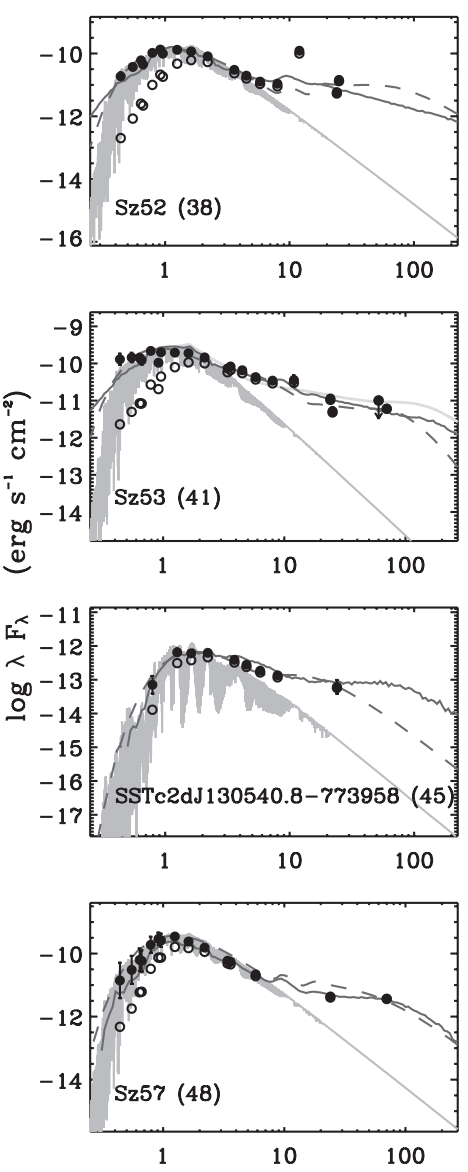
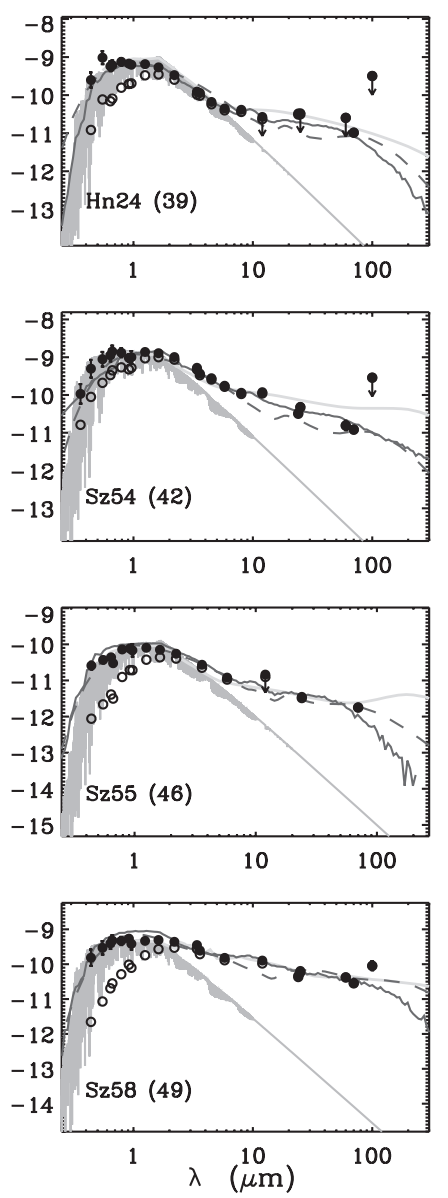

FIG. 7-Continued

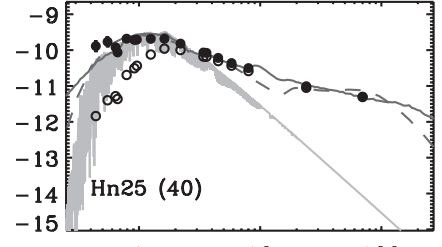

1
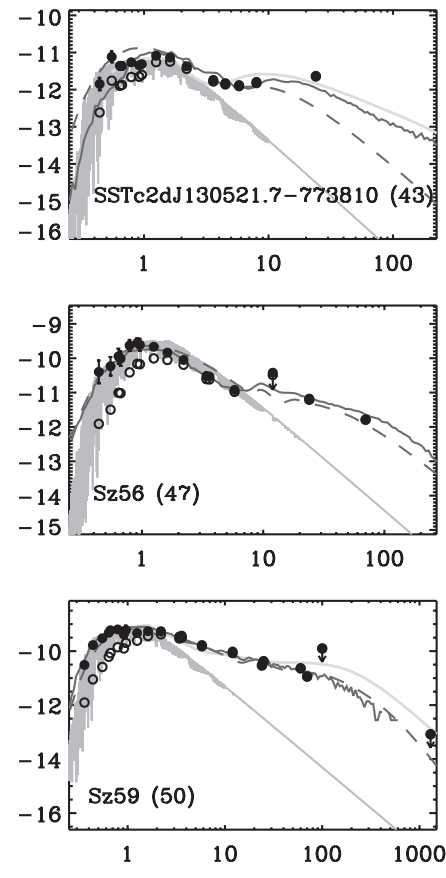

emission of these sources is in the IR, one way to determine their properties is by fitting a disk+envelope accretion model to their SED. From such a fit it is possible to provide an estimate of the temperature and other physical parameters of the central object. This is done in $\S 5.6$.

The observed SEDs of the two Class I objects are presented in Figure 6, while in Figure 7 those for the Class II and Class III objects are shown. The SEDs for the candidate PMS objects are presented in Figure 8. Although the SEDs for a few of these sources have already been presented in previous works that use c2d data (Young et al. 2005; Cieza et al. 2005; Allers et al. 2006; Alcalá et al. 2006; S07a), here we include all of them for completeness. Moreover, we have incorporated in the SEDs additional data in the optical from NOMAD (Zacharias et al. 2004), in the IR from the ISO observations by Persi et al. (2003), and in millimeter wavelength from the observations by Henning et al. (1993). For objects saturated in the $R$ band, we also used the data corresponding to the continuum $\mathrm{H} \alpha$ filter reported in $\mathrm{S} 07 \mathrm{a}$.

Except for a few flat SEDs, most objects in Cha II possess typical SEDs where the flux smoothly drops with wavelength, depending on the Lada class. This may be interpreted in terms of disks that evolve approximately homogeneously from flared to flat (Lada et al. 2006). However, there may be objects in which the SED, after dropping normally to a certain wavelength, rises at longer wavelengths to a flux level comparable to that of flatspectrum or Class II SEDs. An example of such types of objects in our sample is Iso-Cha II 29, with its SED rising beyond $24 \mu \mathrm{m}$ (see Fig. 7). Such SED behavior is explained in terms of an inner disk hole (Lada et al. 2006). These types of sources, better known as transition objects, are interesting because they represent a class of objects where the inner part of the disk may have already been cleared (Calvet et al. 2005 and references therein). The number of these types of objects may depend on the detection limit at $\lambda \geq 24 \mu \mathrm{m}$ (Lada et al. 2006). In Cha II the number statistics are rather poor to make an estimate of their frequency.

\subsection{The $\alpha$ Index and Lada Classes}

The spectral index $\alpha$ can be used to further investigate the different object classes (Lada et al. 2006) in Cha II. Traditionally, this index is determined as the slope of the SED at wavelengths longer than $2 \mu \mathrm{m}$. However, the index may vary significantly depending on the range of wavelengths and method used to derived it. R06 discuss several ways to determine the index and the consequences of using the various combinations of fluxes at different wavelengths. We have followed their prescription in order to determine the index $\alpha_{[K, \text { MIPS } 24 \mu \mathrm{m}]}$ for the Cha II certified and candidate PMS objects. We stress that one of the $\mathrm{c} 2 \mathrm{~d}$ products is the determination and inclusion of the spectral index $\alpha$ in the catalogs. Within the $\mathrm{c} 2 \mathrm{~d}$, the index is calculated as described in the final c2d delivery document (Evans et al. 2007), i.e., the slope of the least-squares fit line to all the flux measurements between the $K$ and MIPS $24 \mu \mathrm{m}$ bands, while $\alpha_{[K, \text { MIPS } 24 \mu \mathrm{m}]}$ is calculated as the slope of the line joining the flux measurements at $K$ and MIPS $24 \mu \mathrm{m}$. We have compared the c2d $\alpha$ index with $\alpha_{[K, \text { MIPS } 24 \mu \mathrm{m}]}$ and find a very good agreement, within an $\mathrm{rms}<0.16$, for all the sources in Cha II detected with $\mathrm{S} / \mathrm{N}>3$ in the bands between $K$ and MIPS $24 \mu \mathrm{m}$. Thus, since the R06 models are used in $\S 5.6$ for the SED fitting, for the sake of homogeneity and for discussions regarding the different Lada classes in Cha II, we use the reference index, $\alpha_{[K, \text { MIPS } 24 \mu \mathrm{m}]}$, as defined by R06, but adopting 

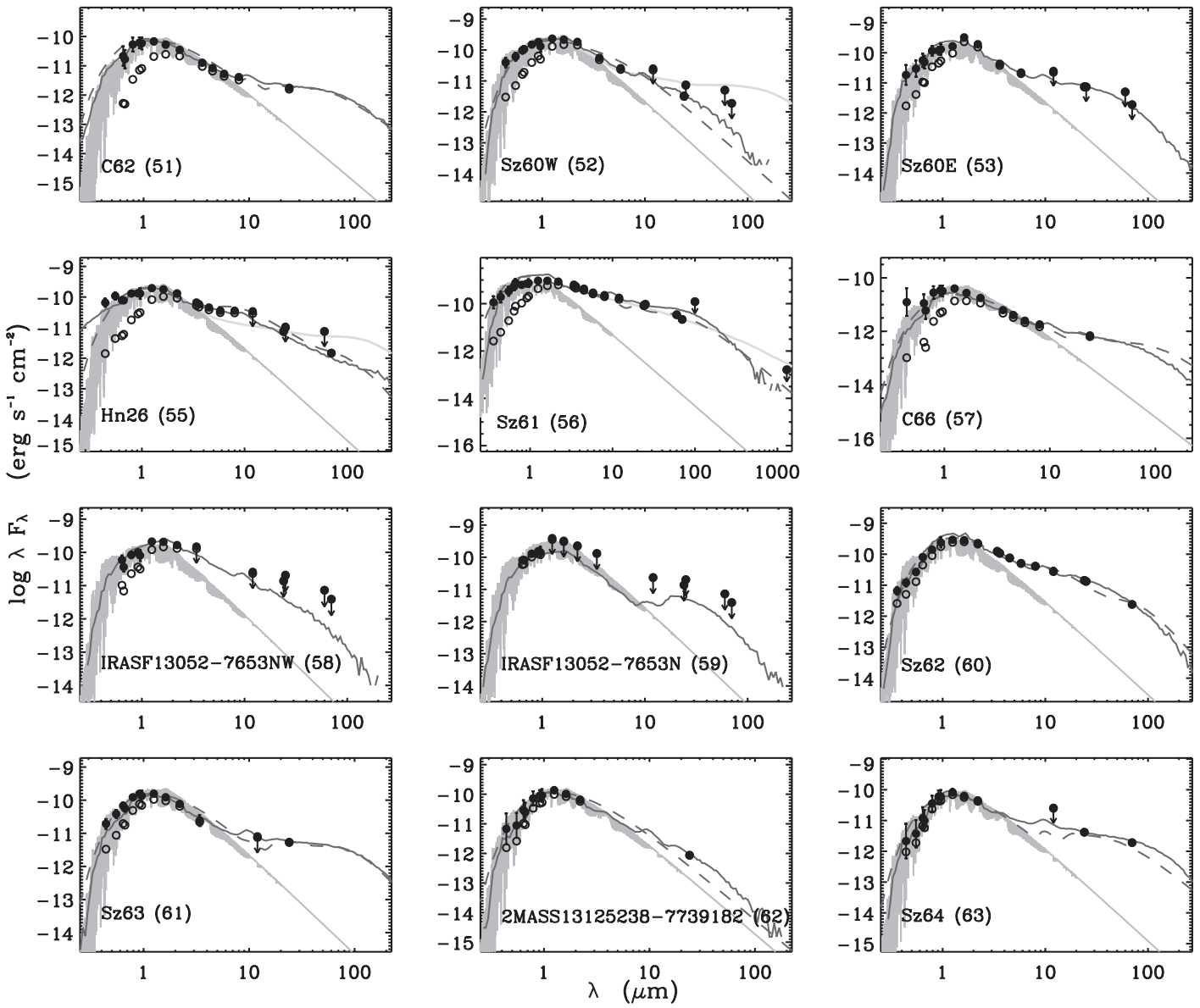

Fig. 7-Continued

the Lada class separation as extended by Greene et al. (1994), i.e., $\alpha_{[K, \text { MIPS } 24 \mu \mathrm{m}]} \geq 0.3$ for Class I, $-0.3 \leq \alpha_{[K, \text { MIPS } 24 \mu \mathrm{m}]}<0.3$ for flat-spectrum sources, $-1.6 \leq \alpha_{[K}$. MIPS $\left.24 \mu \mathrm{m}\right]<-0.3$ for Class II sources, and $\alpha_{[K}$, MIPS $\left.24 \mu \mathrm{m}\right]<-1.6$ for Class III sources. The $\alpha_{[K, \text { MIPS } 24 \mu \mathrm{m}]}$ slope is reported in the third column of Table 8 below.

In Table 6, the statistics of the Lada classes for the YSOs and PMS objects and candidates in Cha II is given. For our purpose, IRAS 13036-7644 (or BHR 86) is included in this table as a Class I source (see Lehtinen et al. 2005). It is interesting to note that including all the PMS objects nearly doubles the number of the Class II sources over the YSOs. The reason is that the IRAC 3.6-8 $\mu \mathrm{m}$ overlap coverage missed about half of the Class II objects in Cha II. Assigning a Lada class to those PMS objects off the IRAC $3.6-8 \mu \mathrm{m}$ overlap area is not a problem because only the $K$ and MIPS $24 \mu$ m fluxes are needed to calculate the $\alpha_{[K, \text { MIPS } 24 \mu \mathrm{m}]}$ index. ${ }^{16}$ Thus, we can classify those too. Only for 2 out of the 62 PMS objects and candidates was it not possible to assign a Lada class because of insufficient photometry (see Tables 2, 3, 4, and 5). One, WFI J12585611-7630105, is a cer-

16 We have, in any case, verified the Class II classification of these objects using IR photometry from the literature and, when available, also the IRAC $3.6 \mu \mathrm{m} /$ IRAC $5.8 \mu \mathrm{m}$ or IRAC $4.5 \mu \mathrm{m} /$ IRAC $8 \mu \mathrm{m}$ data. We remind the reader that, due to the $7^{\prime}$ east-west shift between the IRAC $3.6 \mu \mathrm{m} /$ IRAC $5.8 \mu \mathrm{m}$ and IRAC $4.5 \mu \mathrm{m} /$ IRAC $8 \mu \mathrm{m}$ observations (see end of $\S 3$ and right panel of Fig. 1 by Porras et al. 2007), some objects located off the IRAC 3.6-8 $\mu$ m overlap area, but on the eastern border of the IRAC $3.6 \mu \mathrm{m} /$ IRAC $5.8 \mu \mathrm{m}$ observations, possess only IRAC $3.6 \mu \mathrm{m} /$ IRAC $5.8 \mu \mathrm{m}$ data while others, on the western border of the IRAC $4.5 \mu \mathrm{m} /$ IRAC $8 \mu \mathrm{m}$ observations, possess only IRAC $4.5 \mu \mathrm{m} /$ IRAC $8 \mu \mathrm{m}$ data. tified PMS object, whereas the other, WFI J13031615-7629381, is a candidate PMS object (S07a). Based on their position in the $J-H$ versus $H-K$ diagram, we can classify both objects as Class III. These two objects are, in any case, indicated with question marks in Table 6. Regardless of the inclusion or not of the candidate PMS objects, the dominant objects in Cha II are those of Class II, with only a few Class I sources. Another interesting quantity is the ratio of the number of Class I and flat sources to the number of Class II YSOs and Class III sources; regardless of the inclusion of the candidate PMS objects, this ratio is on the order of 0.1, i.e., a factor of about 2 less than in Serpens (H07). Note that, in addition, some Class III sources in the IRAC 3.6-8 $\mu \mathrm{m}$ /MIPS $24 \mu \mathrm{m}$ overlapping area might be missed by the multicolor selection criteria.

In summary, in Cha II the Class II sources seem to represent $\sim 60 \%$ of the population, the Class III sources $\sim 30 \%$, with only a minority (less than 10\%) being Class I or flat-spectrum sources. These conclusions are in very good agreement with those drawn from the color-color and color-magnitude diagrams reported in $\S 5.1$. We also conclude that in Cha II the Lada classes are quite consistent with the IR stages as defined by R06. Therefore, $\alpha_{[K, \text { MIPS } 24 \mu \mathrm{m}]}=-1.6$ is a good definition for the borderline between optically thick and thin disks in Cha II. This limit is used in $\S 5.9$ to estimate the fraction of optically thick disks.

\subsection{Spatial Distribution and Clustering of the PMS Objects}

The spatial distribution of the different classes of objects in Cha II is shown in Figure 9 overplotted on the c2d extinction map, derived as explained in $\S 6.2$. Note that the latter covers only the 

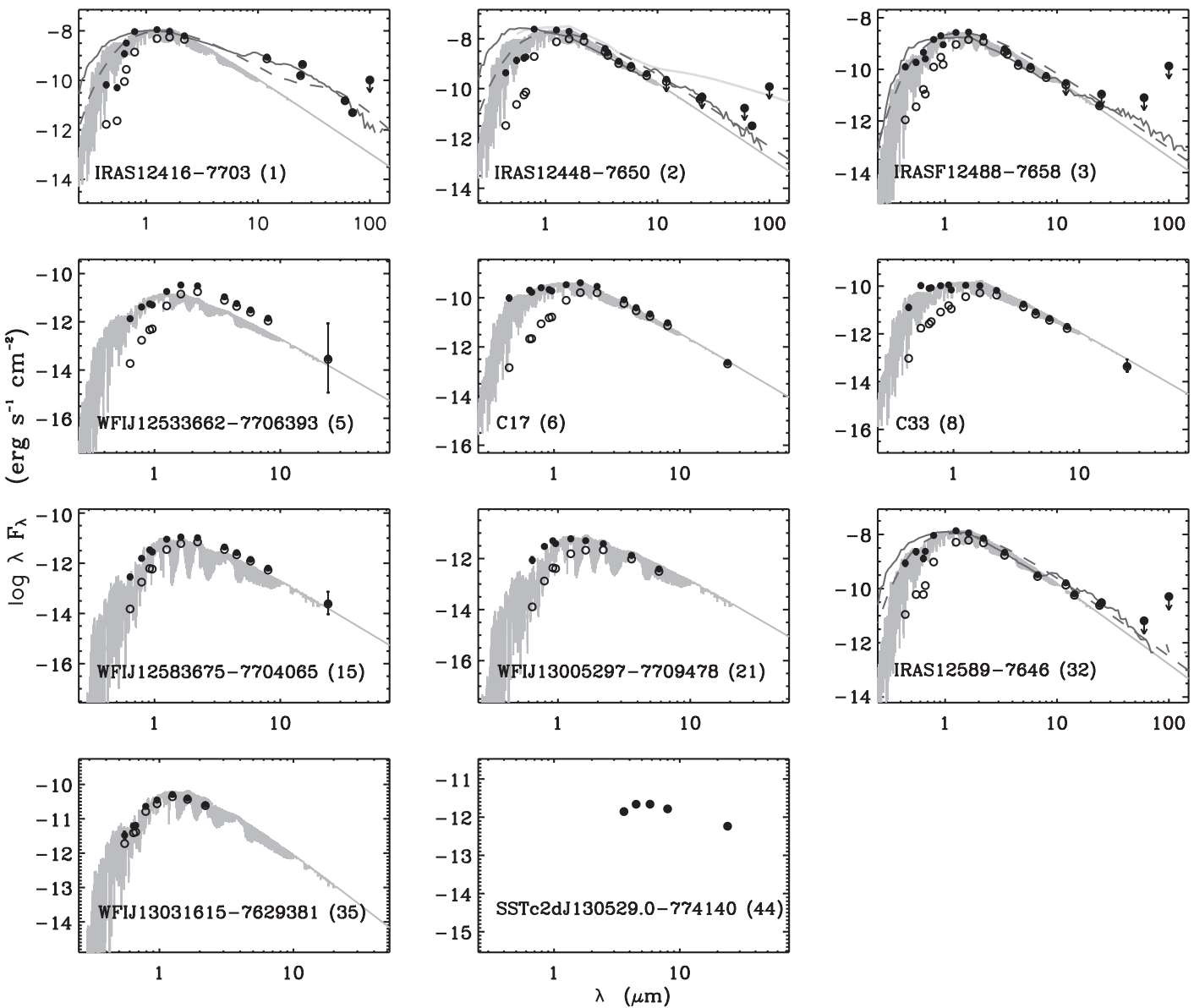

FIG. 8. - SEDs of the candidate PMS objects in Cha II. The symbols are as in Fig. 7. The best-fit NextGen spectrum (by Hauschildt et al. [1999] for objects with $T_{\text {eff }}>4000 \mathrm{~K}$ ) or StarDusty spectrum (by Allard et al. [2000] for objects with $T_{\text {eff }}<4000 \mathrm{~K}$ ) is overplotted on each SED. The best fits of the SEDs derived from both the R06 and D01 models are overplotted as solid and dashed lines, respectively, while those derived from the D'Alessio et al. (2005) models are represented with the thick lines. Fits were not possible for SSTc2d J130529.0-774140 and WFI J13031615-7629381 because of insufficient IR photometry. Likewise, the nondetection of SSTc2d J1305290-774140 in optical bands prevents any estimate of its stellar parameters. The modeling was not attempted for those sources showing pure photospheric Class III SEDs (see $\S 5.6$ ). [See the electronic edition of the Journal for a color version of this figure.]

overlap area observed in all IRAC bands. However, from other maps (Cambrésy 1999) it is evident that there is only one additional extinction peak off the area covered with IRAC, which is close to IRAS 13036-7644 or BHR 86, the easternmost Class I source in Cha II (see Fig. 9). Note that the Class I sources coincide or are located close to the sites of highest extinction.

The arrows in Figure 9 indicate the five optically bright sources IRAS 12416-7703, IRAS 12448-7650, IRAS F12488-7658, IRAS 12556-7731, and IRAS 12589-7646. These sources are scattered over a large area, in regions of quite low extinction. The former four are mainly located to the west of the dust lanes of the main cloud body, while the latter one lies to the east, between the main cloud and BHR 86. As demonstrated in S07b, these five sources are overluminous in the H-R diagram when adopting the
Cha II distance to compute their luminosity and hence might be unrelated to Cha II. However, strong lithium absorption at $6708 \AA$ in the optical spectrum of IRAS 12556-7731 has been detected (S07b); the presence of such a line is a necessary condition to assess the youth of low-mass stars and is used as a diagnostic for the substellar nature of objects below the hydrogen-burning limit. Further discussion on the five IRAS sources will be presented in S07b.

The vast majority of the certified and candidate PMS objects are located at the eastern edge of the cloud, with several of them being off the cloud boundaries as marked by the extinction maps. This may lead to the idea that some agent triggering star formation from the east of the cloud might have occurred. For the Chamaeleon cloud complex there is no evidence of supernova

TABLE 6

Summary of Lada Classes in Cha II

\begin{tabular}{|c|c|c|c|c|c|c|}
\hline Lada Class & Certified YSOs & YSO Candidate & Total YSOs & Certified PMS & PMS Candidate & Total PMS \\
\hline I & 2 & 0 & $2(8 \%)$ & 3 & 0 & $3(5 \%)$ \\
\hline Flat .......................... & 1 & 0 & $1(4 \%)$ & 2 & 0 & $2(3 \%)$ \\
\hline II & 18 & 1 & $19(73 \%)$ & 36 & 2 & $38(61 \%)$ \\
\hline III.............................. & 2 & 2 & $4(15 \%)$ & $9+1 ?$ & $8+1 ?$ & $19(31 \%)$ \\
\hline Total …........................ & 23 & 3 & 26 & 51 & 11 & 62 \\
\hline
\end{tabular}




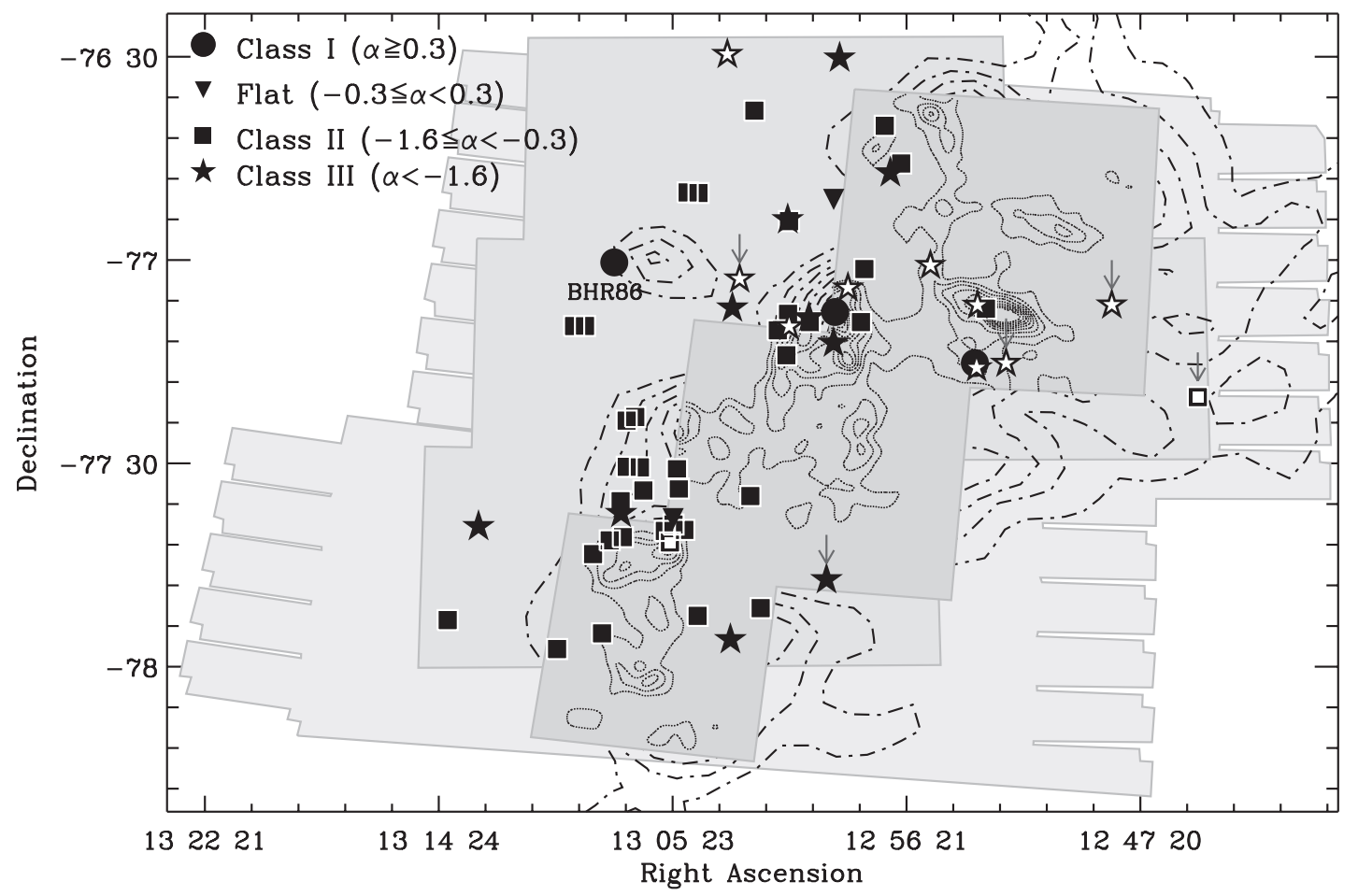

FIG. 9.- Spatial distribution of the certified ( filled symbols) and candidate (open symbols) PMS objects in Cha II as a function of Lada class, overplotted on the contours from the $\mathrm{c} 2 \mathrm{~d}$ extinction map (solid lines; see $\S 6.2$ ). The contour levels of extinction are from 2 to $20 \mathrm{mag}$, in steps of 2 mag. The downward-pointing arrows mark the five optically bright IRAS sources discussed in the text (see $\S$ 5.6). The shaded areas, from light to dark gray, display the regions observed with MIPS, WFI, and the IRAC 3.6-8 $\mu$ m overlap, respectively. The dashed lines outside the IRAC area are the contour levels of extinction from Cambrésy (1999) from 1 to 6 mag in steps of 0.35 mag. The higher resolution of the c2d extinction map with respect to the Cambrésy (1999) map can be appreciated. [See the electronic edition of the Journal for a color version of this figure.]

events or the presence of $\mathrm{OB}$ associations, but a scenario in which a high-velocity cloud with a velocity of about $100 \mathrm{~km} \mathrm{~s}^{-1}$ collides with the Galactic plane at an inclination angle of about $60^{\circ}$ has been proposed by Lepiné \& Duvert (1994). These authors claim that such an event could be responsible for the largescale filamentary structure of the Chamaeleon clouds and hence also of Cha II. It is suspected that the young stars in the cloud complex are moving toward lower Galactic longitudes, approximately in the direction $l=300^{\circ}$ to $l=280^{\circ}$, with a few kilometers per second, almost parallel to the Galactic plane. The spatial distribution of the stars in Chamaeleon shown in Figure 10 by Ducourant et al. (2005) somewhat traces the direction of the velocity vector of the hypothetical high-velocity cloud. Propermotion studies (e.g., Ducourant et al. 2005 and references therein) seem to confirm this, although the errors are quite large and the results may be blurred by the solar reflex motion (Teixeira et al. 2000).

To compare the sample of clouds mapped by $\mathrm{c} 2 \mathrm{~d}$, it is important to examine the distribution of YSOs in a uniform way. Lada \& Lada (2003, hereafter LL03) suggested that a cluster should be a group of some 35 members with a total mass density larger than 1.0 $M_{\odot} \mathrm{pc}^{-3}$. To test this criterion, we calculated the volume density of YSOs for each of the c2d clouds using a nearest neighbor algorithm similar to the one applied by Gutermuth et al. (2005) (for details see J. K. Jørgensen et al. 2008, in preparation), assuming that the distribution of sources is locally spherical. Generally the LL03 criterion is found to be too loose to define substructures and pick out known clusters in the larger scale clouds. We thus adopt the tighter level of 25 times the LL03 criterion (i.e., a mass density of $25 M_{\odot} \mathrm{pc}^{-3}$ ), which normally provides the already established cluster and group boundaries. We refer to "clusters" as regions with more than 35 YSOs within a given volume den- sity contour and "groups" as regions with less. The lowest number of YSOs that is considered to constitute a separate entity is 5 . "Clusters" and "groups" can be either "tight" or "loose" depending on whether their volume densities, $\rho$, are higher than 25 or $1 M_{\odot} \mathrm{pc}^{-3}$ (corresponding to 50 and $2 \mathrm{YSOs} \mathrm{pc}^{-3}$, respectively, assuming an average YSO mass of $0.5 M_{\odot}$ ). We note that although these criteria are useful as a way of making direct comparison between regions within clouds and across different clouds such as those in the c2d sample, this method is empirical and should not be taken, e.g., as evidence in discussions on whether the star formation process is hierarchical or not. For Cha II we applied the algorithm to the whole sample of 62 certified and candidate PMS objects. The overall results, reported in Table 7, are shown in Figure 10. For the sake of comparison with the other c2d clouds, we use the boundaries defined by the whole sample to investigate also the clustering of the 26 YSOs selected from the c2d criteria and also report their statistics in Table 7.

The clustering analysis gives two tight groups in Cha II at the level of 25 times the LL03 criterion; neither have enough members to qualify them as separate clusters. These groups, which we call A and B in Table 7, can be identified by eye in Figure 9. Group A, at around R.A. $=13^{\mathrm{h}} 01^{\mathrm{m}}$ and decl. $=-77^{\circ} 06^{\prime}$, lies close to the position of the Class I source Iso-Cha II 28, while group B, at around R.A. $=13^{\mathrm{h}} 07^{\mathrm{m}}$ and decl. $=-77^{\circ} 35^{\prime}$, is further to the southeast of group A. The two groups apparently coincide with regions of high extinction and several Class II sources are distributed around them, while the Class III sources seem to be more scattered all over the region. On the other hand, Cha II as a whole can be defined as a loose cluster at the level of one times the LL03 criterion. Most of the sources (48 out of $62 ; 75 \%$ ) in Cha II are associated with this cluster, which naturally also includes both the A and B groups from above ( $\frac{1}{3}$ of the cluster 
TABLE 7

Summary of Groups versus Lada Classes in Cha II

\begin{tabular}{|c|c|c|c|c|c|}
\hline Region & Total $\left(N_{\text {YSO }}\right)$ & Class I & Flat & Class II & Class III \\
\hline Cha II loose cluster $(\rho>1 \times$ LL03 $) \ldots \ldots \ldots \ldots \ldots \ldots . . . . . . .$. & $48(22)$ & $2(2)$ & $2(1)$ & $31(17)$ & $13(4)$ \\
\hline 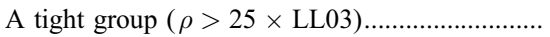 & $6(2)$ & $0(0)$ & $0(0)$ & $4(2)$ & $2(0)$ \\
\hline 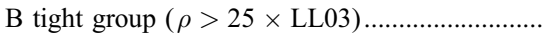 & $10(6)$ & $0(0)$ & $1(1)$ & $8(5)$ & $1(0)$ \\
\hline 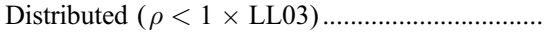 & $14(4)$ & $1(0)$ & $0(0)$ & $7(2)$ & $6(2)$ \\
\hline
\end{tabular}

NOTE.-The number of c2d-identified YSOs is given in parentheses.

members). The overall results are also given in Table 7. At this level, the remaining 14 sources can be considered as part of the extended population in Cha II. Finally, by applying the $1 \times$ LL03 criterion to the restricted YSO sample but using the group boundaries identified from the larger sample of sources, we recover only group B, but with six members.

Interestingly, the Class III sources are more abundant in the loose cluster than in the other substructures. This may lead to the idea that those objects may have formed more closely together and then dispersed during the evolution of the star-forming region. Assuming the typical velocity dispersion of $1 \mathrm{~km} \mathrm{~s}^{-1}$ of young clusters, 2-3 Myr (i.e., the typical age of Cha II members; see S07b) old stars would move about $2 \mathrm{pc}$ from their birth sites. However, the more scattered Class III sources are located at less than $1.5 \mathrm{pc}$ from the tight groups. Therefore, it is more likely that those objects were formed closer to their present position rather than in one of the tight groups.

\subsection{Luminosities}

One way to determine the degree of accretion activity of a PMS population is by means of the frequency of the disk/envelope luminosity of its members. To do this, we calculated the luminosity of each PMS object as follows: (1) The "total" luminosity was

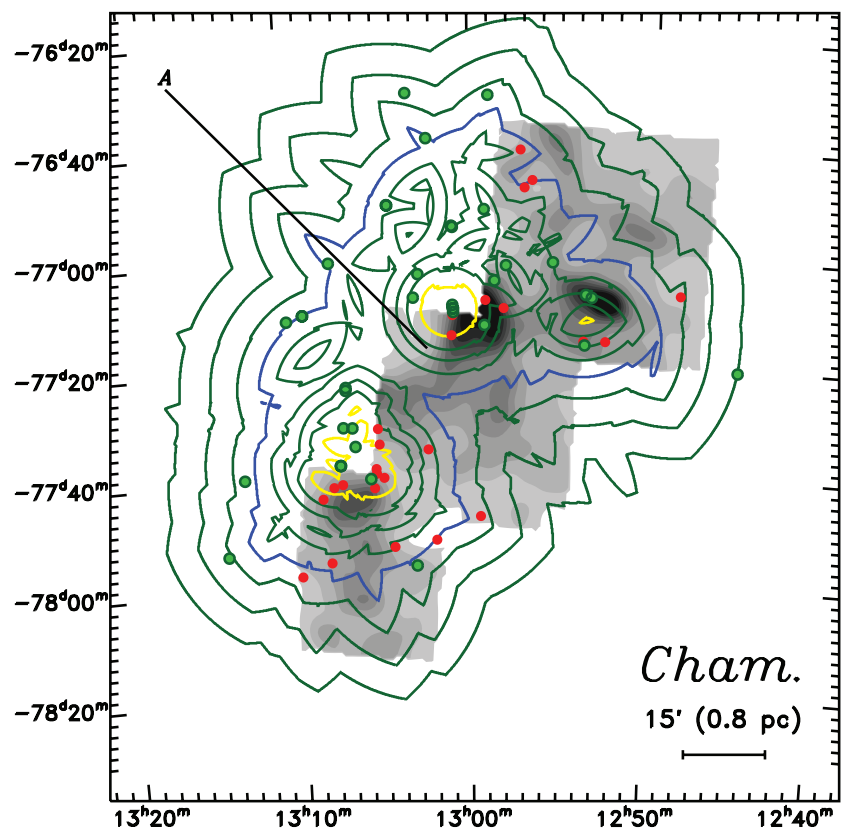

Fig. 10.- Volume density plot of Cha II as determined from the nearest neighbor algorithm described in J. K. Jørgensen et al. (2008, in preparation). The derived density contours are compared with the c2d extinction map (in gray scale). The blue contour corresponds to the $1.0 M_{\odot} \mathrm{pc}^{-3}$ density level, while the green ones correspond to levels of $0.125,0.25,0.5,2,4$, and 8 times this level. The yellow contour corresponds to the $25 M_{\odot} \mathrm{pc}^{-3}$ density level. The two tight groups in Cha II are marked. Red filled circles represent the YSOs, while the green filled circles represent the rest of the PMS objects. first computed by direct integration of its dereddened SED, assuming isotropic radiation and the distance $d=178 \mathrm{pc}$ (Whittet et al. 1997); we have extrapolated the SED beyond the available terminal wavelength using the method by Cohen (1973). (2) For each PMS object the stellar luminosity was also computed by integrating the model spectrum (Allard \& Hauschildt 1995; Allard et al. 2000) corresponding to the PMS object temperature or, in the case of the candidate PMS objects, to the model spectrum that best fits the dereddened SED as explained in S07a.

Using the total luminosity calculated as explained in item 1 above, we have investigated the expected number of low-luminosity objects and completeness in the IRAC 3.6-8 $\mu \mathrm{m} /$ MIPS $24 \mu \mathrm{m}$ overlap area in Cha II. To this aim, we explored similar ideas to those exposed in $\mathrm{H} 07$ regarding luminosity histograms and completeness limits in the $1-30 \mu \mathrm{m}$ range. Since the full SWIRE catalog is much deeper than the $\mathrm{c} 2 \mathrm{~d}$ one, it can be considered as a reference catalog with $100 \%$ completeness. Both the full SWIRE catalog and a "trimmed" version of it, which matches the sensitivity of the c2d observations in Cha II, are thus used for completeness determinations. In Figure 11 two luminosity histograms are shown: one for the YSOs, while the other one is the luminosity histogram corrected for completeness according to the guidelines described in H07. Both histograms are produced assuming the distance of $178 \mathrm{pc}$ for Cha II. The luminosity histograms show that only a very small number $(\sim 2)$ of additional low-luminosity $\left[\log \left(L / L_{\odot}\right)<-1.7\right]$ YSOs are still expected to be found. Therefore, we conclude that the sample of YSO candidates in Cha II, as provided by the observations in the IRAC 3.6-8 $\mu \mathrm{m} /$ MIPS $24 \mu \mathrm{m}$

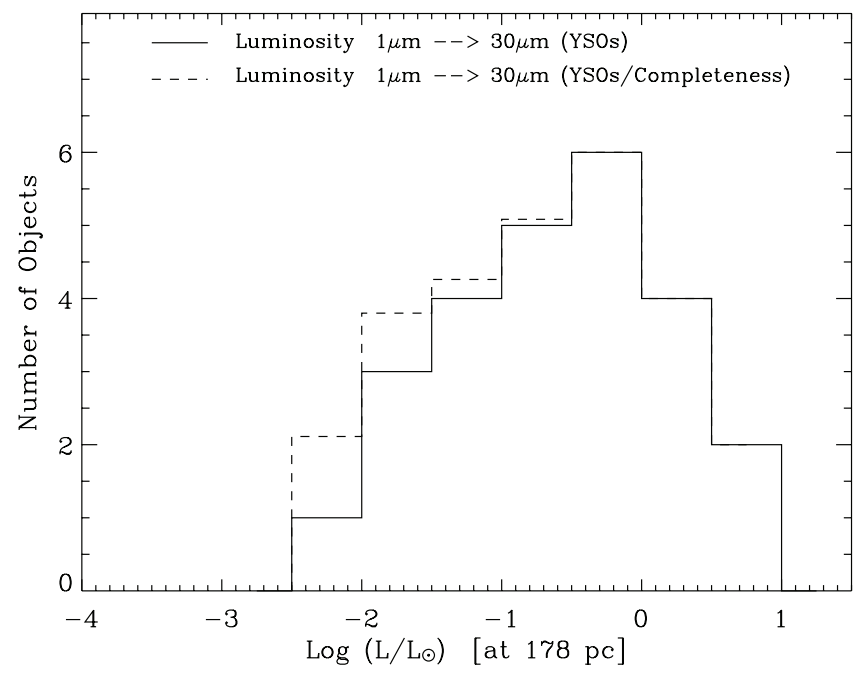

FIG. 11.-Luminosity distribution for the YSOs in the overlapping area surveyed with IRAC and MIPS in Cha II (solid histogram). The plotted luminosities were determined as in H07, i.e., by integration of the SEDs from 1 to $30 \mu \mathrm{m}$. The corrected luminosity distribution, determined by applying completeness factors at each luminosity bin as in H07, is overplotted (dashed histogram). Note that IRAS $12496-7650$ is not considered in these histograms because it is saturated in IRAC 3.6 and $4.5 \mu \mathrm{m}$ (see also $\S 3.1$ ). 


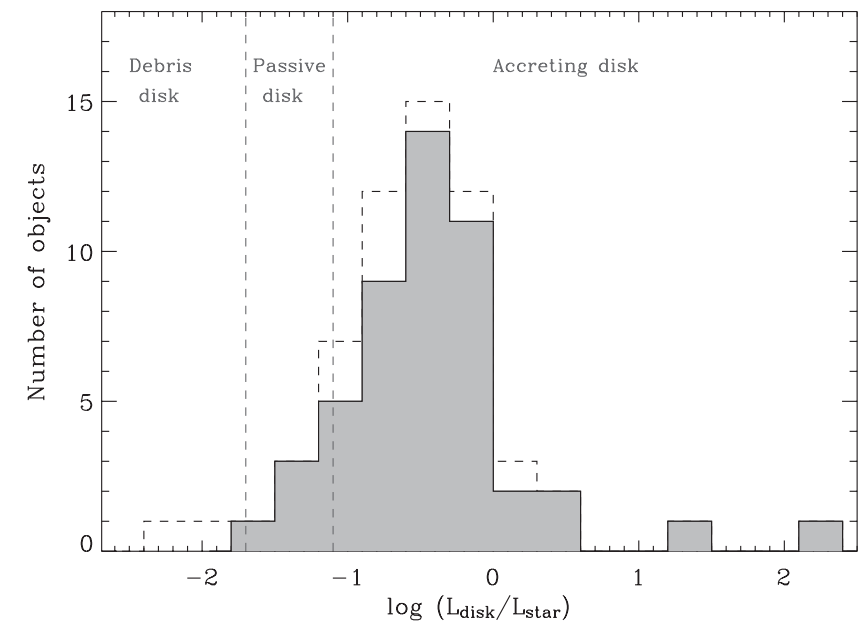

FIG. 12.-Distribution of the $L_{\text {disk }} / L_{\text {star }}$ ratio of the confirmed PMS objects (solid histogram) and when adding the candidate PMS objects (dashed histogram). The dashed vertical lines mark the typical boundaries of active, passive, and debris disks as defined by Kenyon \& Hartmann (1987). Most objects in Cha II are in the accretion phase. [See the electronic edition of the Journal for a color version of this figure.]

overlap area, is fairly complete. We recall, in addition, that the two very low luminosity objects discovered by Allers et al. (2006) were not selected from the multicolor criteria by $\mathrm{H} 07$ (see Table 1) because they are too faint in MIPS $24 \mu \mathrm{m} .{ }^{17}$ Hence, they are not among the objects producing the solid histogram in Figure 11. These two objects are, in any case, included in our census, making our sample of YSOs complete down to $\log \left(L / L_{\odot}\right) \approx-2.5$. Although slightly higher, this limit is consistent with the one derived from the limiting magnitudes for a complete sample in Cha II, based on the histograms of source number counts, reported in the basic data papers by Porras et al. (2007) and Young et al. (2005) for the IRAC and MIPS observations, respectively.

In order to study the excess luminosity, we compare the total luminosity with the one emitted only by the central object: the difference between the two luminosities derived as in items 1 and 2 above can be attributed to the circumstellar material. Figure 12 shows the distribution of the residual luminosity, i.e., of the $L_{\text {disk }} /$ $L_{\text {star }}$ ratio, for the Cha II certified and candidate PMS objects. From this figure it is evident that the Cha II population is dominated by objects with active accretion, in agreement with the conclusions of $\S 5.3$. It is also evident that the average PMS object in Cha II is the one for which the disk luminosity is about one-third of the stellar luminosity. In the case of the Class I sources we have derived the $L_{\text {disk+envelope }} / L_{\text {star }}$ ratio after the SED model fits performed in $\S 5.6$. The models provide the approximate temperature of the central object from which it is possible to derive a luminosity.

\subsection{Disk Modeling}

Understanding the evolution of accretion disks can provide strong constraints on theories of planet formation and evolution (Lynden-Bell \& Pringle 1974; Adams et al. 1987; Kenyon \& Hartmann 1987; Backman \& Paresce 1993; Lagrange et al. 2000; Zuckerman 2001; Dullemond et al. 2007 and references therein), and measuring the lowest mass at which young objects harbor circumstellar disks is crucial for determining whether planets can form around low-mass BDs (Luhman et al. 2005a, 2005c; Lada et al. 2006).

\footnotetext{
${ }^{17}$ MIPS $24 \mu \mathrm{m}$ fluxes for these two objects are provided by the band-filling process, but errors are too large to be accepted by the $\mathrm{H} 07$ criteria.
}

In order to further investigate the circumstellar material of the Cha II PMS objects, we have modeled the SEDs using the Dullemond et al. (2001, hereafter D01), D'Alessio et al. (2005), and R06 SED models. While the D01 models consider reprocessing flared disks, D'Alessio et al. (2005) and R06 simulate the SEDs of PMS stars with different combinations of accretion disk/envelope parameters. The sampling of the parameter space is quite different among the three prescriptions. For instance, in the D'Alessio et al. (2005) models the central object temperature is in steps of $500 \mathrm{~K}$, with a minimum star temperature of $4000 \mathrm{~K}$ and a minimum stellar mass of $0.7 M_{\odot}$; in addition, such models are provided only for two disk inclination angles $\left(30^{\circ}\right.$ and $\left.60^{\circ}\right)$. The sampling is much better in the D01 and R06 model grids, but the minimum stellar mass for which the R06 models are valid is $0.1 M_{\odot}$; thus, for objects far below this, the modeling results must be treated with caution. Flux upper limits are taken into account in the SED fitting tool by Robitaille et al. (2007, hereafter R07), while in the D01 and D'Alessio et al. (2005) prescriptions no upper limits are considered; however, when using the D01 and D'Alessio et al. (2005) models, we checked the consistency between the best-fit model and possible flux upper limits.

Taking all this into account, the SED fits were performed using the following criteria:

1. The models are done for star+disk/envelope systems; thus, SED fits of pure photospheric Class III SEDs cannot be attempted.

2. SEDs with only flux upper limits in their IR part were fitted using only the R06 models. The D01 and D'Alessio et al. (2005) models were fitted only if a reasonable number of IR absolute fluxes are available.

3. For the fits, the temperature of the central object was fixed using the values derived in S07b. ${ }^{18}$ The D'Alessio et al. (2005) model fits were attempted only for objects with $T_{\text {eff }}>3500 \mathrm{~K}$, selecting the model object temperature closest to the one provided in $\mathrm{S} 07 \mathrm{~b}$.

4. The fits of the D'Alessio et al. (2005) models were performed by normalizing the modeled SEDs to the derreddened I-band flux, where the possibility of excess is minimized.

5. The fits of the Class I SEDs were done using only the R06 models.

6. For objects not detected in the optical, like the Class I source Iso-Cha II 28, we have set all R07 input parameters free.

In Figures 6, 7, and 8 the resulting best fits are overplotted as solid and dashed lines for the R06 and D01 models, respectively, while those derived from the D'Alessio et al. (2005) models are represented with thick lines. The general results are reported in Tables 8 and 9 . We caution that the results on the mass accretion rate are not reliable for Class II sources lacking $U$ or $B$ photometry. Therefore, this parameter is provided only for such objects possessing fluxes in at least one of these two bands. For some of these objects the resulting mass accretion rate is zero. We also caution that the output disk parameters refer to the best-fit model SED to each source and that there may be a number of model SEDs providing a good fit. In this sense, the parameter values quoted in Tables 8 and 9 should be treated with caution.

A reasonable fit with the R06 models could be performed basically for all the sources with IR excess in the sample. Exceptions are the strongly veiled object $\mathrm{Sz} 47$ and the YSO candidate SSTc2d J130529.0-774140, the latter with insufficient photometry for the analysis. Noteworthy are the results for the Class I

18 Fixing the star temperature is not foreseen in the R07 Web interface. Thus, an IDL procedure was used that reads the grid of output models and selects the one that, matching input stellar parameters, best fits the SED. 
TABLE 8

SED Parameters and Disk Modeling Results from D01 Models

\begin{tabular}{|c|c|c|c|c|c|c|c|c|c|}
\hline Number & Object ID & $\alpha_{[K, \operatorname{MIPS} 24 \mu \mathrm{m}]}$ & $\log \left(L_{d} / L_{*}\right)$ & $\begin{array}{c}\log L_{d} \\
\left(L_{\odot}\right)\end{array}$ & $\begin{array}{c}\lambda_{\text {turnoff }} \\
(\mu \mathrm{m})\end{array}$ & $\alpha_{\text {excess }}$ & $\begin{array}{l}R_{\text {disk }}^{\text {Dull }} \\
(\mathrm{AU})\end{array}$ & $\begin{array}{l}R_{\mathrm{in}}^{\text {Dull }} \\
(\mathrm{AU})\end{array}$ & $\begin{array}{c}\log M_{\text {disk }}^{\text {Dull }} \\
\left(M_{\odot}\right)\end{array}$ \\
\hline $1 \ldots \ldots \ldots \ldots \ldots \ldots$ & IRAS $12416-7703$ & -1.51 & -0.90 & 0.21 & 2.2 & -1.64 & 15.0 & 0.24 & -3.7 \\
\hline $3 \ldots \ldots \ldots \ldots \ldots \ldots \ldots$ & IRAS F12488-7658 & -2.54 & -0.75 & -0.32 & 8.0 & -2.24 & 60.0 & 0.08 & -7.0 \\
\hline $4 \ldots \ldots \ldots \ldots \ldots \ldots \ldots$ & IRAS 12496-7650 & -0.72 & 0.36 & 1.63 & 0.9 & 0.05 & 200.0 & 1.30 & -0.4 \\
\hline $5 \ldots \ldots \ldots \ldots \ldots \ldots \ldots$ & WFI J12533662-7706393 & -2.88 & 0.26 & -1.69 & - & - & - & - & - \\
\hline $6 \ldots \ldots \ldots \ldots \ldots \ldots \ldots$ & $\mathrm{C} 17$ & -2.98 & -1.15 & -1.49 & - & - & - & - & - \\
\hline $8 \ldots \ldots \ldots \ldots \ldots \ldots \ldots$ & $\mathrm{C} 33$ & -3.03 & -0.77 & -1.55 & - & - & - & - & - \\
\hline $9 \ldots \ldots \ldots \ldots \ldots \ldots \ldots$ & IRAS $12522-7640$ & & - & - & - & - & - & - & - \\
\hline $10 \ldots \ldots \ldots \ldots \ldots \ldots$ & $\mathrm{Sz} 46 \mathrm{~N}$ & -1.27 & -0.94 & -1.43 & 2.2 & -1.29 & 3.0 & 0.05 & -2.3 \\
\hline $11 \ldots \ldots \ldots \ldots \ldots \ldots$ & Sz 47 & -2.06 & - & - & - & - & - & - & - \\
\hline $12 \ldots \ldots \ldots \ldots \ldots \ldots$ & IRAS $12535-7623$ & -0.98 & -0.47 & -0.33 & 2.2 & -0.98 & 20.0 & 0.15 & -1.8 \\
\hline $13 \ldots \ldots \ldots \ldots \ldots \ldots$ & SSTc2d J125758.7-770120 & -1.10 & -0.48 & -3.52 & 5.8 & -0.57 & 0.5 & 0.01 & -3.3 \\
\hline $14 \ldots \ldots \ldots \ldots \ldots \ldots$ & Iso-Cha II 13 & -1.06 & 0.23 & -1.82 & 1.2 & -1.03 & 0.4 & 0.01 & -3.8 \\
\hline $19 \ldots \ldots \ldots \ldots \ldots \ldots$ & Iso-Cha II 29 & -2.86 & -0.78 & -0.97 & 24.0 & 1.76 & 50.0 & 0.05 & -7.0 \\
\hline $20 \ldots \ldots \ldots \ldots \ldots \ldots$ & IRAS 12556-7731 & -1.90 & -1.16 & -0.08 & 8.0 & -1.07 & 100.0 & 0.12 & -2.4 \\
\hline $21 \ldots \ldots \ldots \ldots \ldots \ldots$ & WFI J13005297-7709478 & -2.06 & -1.15 & -3.22 & - & - & - & - & - \\
\hline $22 \ldots \ldots \ldots \ldots \ldots \ldots$ & $\mathrm{Sz} 48 \mathrm{NE}$ & -1.00 & -0.36 & -0.83 & 2.2 & $-1.27^{\mathrm{b}}$ & - & - & - \\
\hline $23 \ldots \ldots \ldots \ldots \ldots \ldots$ & Sz 49 & -0.61 & -0.47 & -1.17 & 3.4 & -0.43 & 5.0 & 0.05 & -1.3 \\
\hline $24 \ldots \ldots \ldots \ldots \ldots \ldots$ & Sz 48SW & -0.95 & -0.16 & -0.74 & 2.2 & $-1.35^{\mathrm{b}}$ & - & - & - \\
\hline $25 \ldots \ldots \ldots \ldots \ldots \ldots$ & $\mathrm{Sz} 50$ & -0.88 & -0.71 & -0.64 & 4.5 & -0.50 & 6.0 & 0.62 & -1.0 \\
\hline $26 \ldots \ldots \ldots \ldots \ldots \ldots$ & WFI J13005531-7708295 & -2.87 & -1.53 & -1.98 & - & - & - & - & - \\
\hline $27 \ldots \ldots \ldots \ldots \ldots \ldots$ & RX J1301.0-7654a & -2.82 & -1.27 & -0.89 & - & - & - & - & - \\
\hline $28 \ldots \ldots \ldots \ldots \ldots \ldots$ & IRAS F12571-7657 & -1.19 & 0.32 & 0.17 & 0.9 & -1.24 & 4.0 & 0.10 & -2.3 \\
\hline $29 \ldots \ldots \ldots \ldots \ldots$ & Sz 51 & -1.12 & -0.09 & -0.45 & 2.2 & -1.07 & 10.0 & 0.11 & -2.3 \\
\hline $30 \ldots \ldots \ldots \ldots \ldots \ldots$ & CM Cha & -0.95 & -0.39 & -0.53 & 3.4 & -0.39 & 15.0 & 0.59 & -2.0 \\
\hline $31 \ldots \ldots \ldots \ldots \ldots \ldots$ & $\mathrm{C} 50$ & -1.50 & -1.07 & -2.26 & 5.8 & -1.11 & 3.0 & 0.02 & -4.0 \\
\hline $40 \ldots \ldots \ldots \ldots \ldots \ldots$ & Hn 25 & -1.14 & -0.68 & -1.17 & 2.2 & -1.09 & 40.0 & 0.06 & -3.0 \\
\hline $41 \ldots \ldots \ldots \ldots \ldots \ldots$ & $\mathrm{Sz} 53$ & -1.07 & -0.77 & -1.26 & 2.2 & -1.01 & 30.0 & 0.14 & -3.0 \\
\hline $42 \ldots \ldots \ldots \ldots \ldots \ldots$ & $\mathrm{Sz} 54$ & -1.41 & -0.45 & -0.15 & 1.6 & -1.33 & 20.0 & 0.12 & -1.3 \\
\hline $43 \ldots \ldots \ldots \ldots \ldots \ldots$ & SSTc2d J130521.7-773810 & -0.26 & -0.13 & -2.17 & 2.2 & -0.29 & 70.0 & 0.13 & -4.0 \\
\hline $44 \ldots \ldots \ldots \ldots \ldots \ldots$ & SSTc2d J130529.0-774140 & -0.37 & - & - & - & - & - & - & - \\
\hline $45 \ldots \ldots \ldots \ldots \ldots \ldots$ & SSTc2d J130540.8-773958 & -0.97 & -0.08 & -3.30 & 3.6 & -1.32 & 0.5 & 0.05 & -5.0 \\
\hline $46 \ldots \ldots \ldots \ldots \ldots \ldots$ & Sz 55 & -1.19 & -0.87 & -1.77 & 3.6 & -1.05 & 3.0 & 0.04 & -1.3 \\
\hline $47 \ldots \ldots \ldots \ldots \ldots \ldots$ & Sz 56 & -1.08 & -0.58 & -1.05 & 5.8 & -0.57 & 1.0 & 0.02 & -3.3 \\
\hline $48 \ldots \ldots \ldots \ldots \ldots \ldots$ & Sz 57 & -1.55 & -0.63 & -1.03 & 5.8 & -0.89 & 2.0 & 0.02 & -3.0 \\
\hline $49 \ldots \ldots \ldots \ldots \ldots \ldots$ & $\mathrm{Sz} 58$ & -0.93 & -0.01 & -0.18 & 1.6 & -0.75 & 100.0 & 0.24 & -0.7 \\
\hline $50 \ldots \ldots \ldots \ldots \ldots \ldots$ & Sz 59 & -1.20 & -0.25 & -0.30 & 1.6 & -1.05 & 20.0 & 0.23 & -2.0 \\
\hline $51 \ldots \ldots \ldots \ldots \ldots$ & C62 & -1.28 & -0.91 & -1.96 & 5.8 & -0.77 & 1.0 & 0.02 & -3.0 \\
\hline $52 \ldots \ldots \ldots \ldots \ldots$ & $\mathrm{Sz} 60 \mathrm{~W}$ & -1.67 & -0.79 & -1.33 & 2.2 & -1.74 & 20.0 & 0.09 & -3.3 \\
\hline $53 \ldots \ldots \ldots \ldots \ldots$ & $\mathrm{Sz} 60 \mathrm{E}$ & -1.35 & -0.35 & -1.03 & 3.6 & -0.93 & - & - & - \\
\hline $54 \ldots \ldots \ldots \ldots \ldots \ldots$ & IRAS $13036-7644$ & & - & - & - & - & - & - & - \\
\hline $55 \ldots \ldots \ldots \ldots \ldots \ldots$ & Hn 26 & -1.17 & -0.70 & -1.29 & 2.2 & -1.12 & 20.0 & 0.32 & -3.0 \\
\hline $56 \ldots \ldots \ldots \ldots \ldots$ & Sz 61 & -0.97 & -0.11 & -0.04 & 1.6 & -0.99 & 10.0 & 0.26 & -0.8 \\
\hline $57 \ldots \ldots \ldots \ldots \ldots$ & C66 & -1.32 & -1.04 & -2.34 & 5.8 & -0.90 & 2.0 & 0.01 & -4.0 \\
\hline $58 \ldots \ldots \ldots \ldots \ldots \ldots$ & IRAS F13052-7653NW & -1.07 & -0.01 & -0.71 & 1.6 & -0.94 & - & - & - \\
\hline $59 \ldots \ldots \ldots \ldots \ldots \ldots$ & IRAS F13052-7653N & -1.17 & -0.42 & -0.89 & 2.2 & $-1.09^{\mathrm{b}}$ & - & - & - \\
\hline 60..................... & Sz 62 & -1.16 & -0.50 & -0.98 & 2.2 & -1.27 & 200.0 & 0.66 & -4.0 \\
\hline 61.................... & Sz 63 & -1.11 & -1.44 & -2.09 & - & - & 60.0 & 0.02 & -3.3 \\
\hline $62 \ldots \ldots \ldots \ldots \ldots \ldots$ & 2MASS $13125238-7739182$ & -1.80 & -0.41 & -1.22 & 2.2 & -1.80 & 50.0 & 0.03 & -5.0 \\
\hline $63 \ldots \ldots \ldots \ldots \ldots \ldots$ & Sz 64 & -1.10 & -0.09 & -1.14 & 2.2 & -1.07 & 1.0 & 0.01 & -2.3 \\
\hline
\end{tabular}

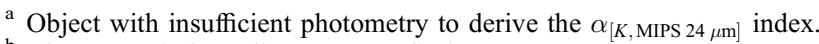

b The $\alpha_{\text {excess }}$ index relies on upper limits. 
TABLE 9

Disk Modeling Results from R07 and D’ Alessio et Al. (2005) Models

\begin{tabular}{|c|c|c|c|c|c|c|c|c|c|}
\hline Number & Object ID & $\begin{array}{l}R_{\text {disk }}^{\text {Rob }} \\
(\mathrm{AU})\end{array}$ & $\begin{array}{l}R_{\text {in }}^{\text {Rob }} \\
(\mathrm{AU})\end{array}$ & $\begin{array}{c}\log M_{\text {disk }}^{\text {Rob }} \\
\left(M_{\odot}\right)\end{array}$ & $\begin{array}{c}M_{\mathrm{accr}}^{\mathrm{Roba}} \\
\left(M_{\odot} \mathrm{yr}^{-1}\right)\end{array}$ & $\begin{array}{l}R_{\text {disk }}^{\mathrm{D}^{\prime} \mathrm{Ale}} \\
(\mathrm{AU})\end{array}$ & $\begin{array}{l}R_{\mathrm{in}}^{\mathrm{D}^{\prime} \mathrm{Ale}} \\
(\mathrm{AU})\end{array}$ & $\begin{array}{c}\log M_{\text {disk }}^{\mathrm{D}^{\prime} \mathrm{Ale}} \\
\left(M_{\odot}\right)\end{array}$ & $\begin{array}{c}M_{\mathrm{accr}}^{\mathrm{D}^{\prime} \mathrm{Alea}} \\
\left(M_{\odot} \mathrm{yr}^{-1}\right)\end{array}$ \\
\hline $1 \ldots \ldots \ldots \ldots \ldots \ldots \ldots \ldots$ & IRAS 12416-7703 & 42 & 0.43 & -5.56 & $0.0 \mathrm{E}+00$ & $\ldots$ & $\ldots$ & $\ldots$ & $\ldots$ \\
\hline $2 \ldots \ldots \ldots \ldots \ldots \ldots \ldots \ldots$ & IRAS $12448-7650$ & 263 & 0.40 & -6.31 & $1.0 \mathrm{E}-08$ & 100 & 0.11 & -3.00 & 1.E-09 \\
\hline $3 \ldots \ldots \ldots \ldots \ldots \ldots \ldots$ & IRAS F12488-7658 & 1805 & 0.13 & -6.63 & $3.6 \mathrm{E}-08$ & $\ldots$ & $\ldots$ & $\ldots$ & $\ldots$ \\
\hline $4 \ldots \ldots \ldots \ldots \ldots \ldots \ldots$ & IRAS $12496-7650$ & 149 & 1.79 & -2.90 & $1.8 \mathrm{E}-07$ & 800 & 1.01 & 0.93 & 1.E-06 \\
\hline $5 \ldots \ldots \ldots \ldots \ldots \ldots \ldots$ & WFI J12533662-7706393 & $\ldots$ & $\ldots$ & $\ldots$ & $\ldots$ & $\ldots$ & $\ldots$ & $\ldots$ & $\ldots$ \\
\hline 6........................ & C17 & $\cdots$ & $\ldots$ & $\cdots$ & $\cdots$ & $\cdots$ & $\cdots$ & $\cdots$ & $\cdots$ \\
\hline 7.......................... & IRAS $12500-7658$ & 50 & 0.65 & -2.19 & $2.0 \mathrm{E}-06$ & $\ldots$ & $\ldots$ & $\ldots$ & $\ldots$ \\
\hline $8 \ldots \ldots \ldots \ldots \ldots \ldots \ldots$ & C33 & $\cdots$ & $\ldots$ & $\ldots$ & $\ldots$ & $\ldots$ & $\ldots$ & $\ldots$ & $\ldots$ \\
\hline $9 \ldots \ldots \ldots \ldots \ldots \ldots \ldots$ & IRAS $12522-7640$ & $\ldots$ & $\ldots$ & $\cdots$ & $\cdots$ & $\cdots$ & $\cdots$ & $\cdots$ & $\cdots$ \\
\hline $10 \ldots \ldots \ldots \ldots \ldots \ldots$ & $\mathrm{Sz} 46 \mathrm{~N}$ & 137 & 0.13 & -4.72 & $1.1 \mathrm{E}-07$ & 100 & 0.06 & -1.39 & 1.E-08 \\
\hline $11 \ldots \ldots \ldots \ldots \ldots \ldots$ & Sz 47 & $\ldots$ & $\ldots$ & $\ldots$ & $\ldots$ & $\ldots$ & $\ldots$ & $\ldots$ & $\ldots$ \\
\hline $12 \ldots \ldots \ldots \ldots \ldots \ldots$ & IRAS $12535-7623$ & 75 & 0.49 & -3.60 & $2.1 \mathrm{E}-08$ & 300 & 0.06 & -1.18 & 1.E-08 \\
\hline $13 \ldots \ldots \ldots \ldots \ldots \ldots$ & SSTc2d J125758.7-770120 & 237 & 0.01 & -3.09 & $\ldots$ & $\ldots$ & $\ldots$ & $\ldots$ & $\ldots$ \\
\hline $14 \ldots \ldots \ldots \ldots \ldots \ldots$ & Iso-Cha II 13 & 116 & 0.19 & -5.74 & $\ldots$ & $\ldots$ & $\ldots$ & $\ldots$ & $\ldots$ \\
\hline $15 \ldots \ldots \ldots \ldots \ldots \ldots$ & WFI J12583675-7704065 & $\cdots$ & $\cdots$ & $\cdots$ & $\cdots$ & $\cdots$ & $\cdots$ & $\cdots$ & $\cdots$ \\
\hline $16 \ldots \ldots \ldots \ldots \ldots \ldots$ & WFI J12585611-7630105 & $\ldots$ & $\ldots$ & $\ldots$ & $\ldots$ & $\ldots$ & $\ldots$ & $\ldots$ & $\ldots$ \\
\hline $17 \ldots \ldots \ldots \ldots \ldots \ldots$ & Iso-Cha II 28 & 130 & 0.25 & -1.26 & $5.7 \mathrm{E}-07$ & $\ldots$ & $\ldots$ & $\ldots$ & $\ldots$ \\
\hline $18 \ldots \ldots \ldots \ldots \ldots \ldots$ & $\mathrm{C} 41$ & 973 & 0.98 & -2.42 & - & $\ldots$ & $\ldots$ & $\ldots$ & $\ldots$ \\
\hline $19 \ldots \ldots \ldots \ldots \ldots \ldots$ & Iso-Cha II 29 & 522 & 70.86 & -5.81 & $2.2 \mathrm{E}-09$ & 300 & 0.11 & -2.40 & 1.E-09 \\
\hline $20 \ldots \ldots \ldots \ldots \ldots \ldots$ & IRAS $12556-7731$ & 320 & 0.22 & -5.97 & $6.0 \mathrm{E}-09$ & $\ldots$ & $\ldots$ & $\ldots$ & $\ldots$ \\
\hline $21 \ldots \ldots \ldots \ldots \ldots \ldots$ & WFI J13005297-7709478 & $\ldots$ & $\ldots$ & $\ldots$ & $\ldots$ & $\ldots$ & $\ldots$ & $\ldots$ & $\ldots$ \\
\hline $22 \ldots \ldots \ldots \ldots \ldots \ldots \ldots$ & $\mathrm{Sz} 48 \mathrm{NE}$ & 206 & 0.53 & -6.23 & $0.0 \mathrm{E}+00$ & $\ldots$ & $\cdots$ & $\ldots$ & $\ldots$ \\
\hline $23 \ldots \ldots \ldots \ldots \ldots \ldots$ & Sz 49 & 66 & 0.03 & -2.61 & $4.8 \mathrm{E}-10$ & 300 & 0.06 & -1.11 & 1.E-08 \\
\hline $24 \ldots \ldots \ldots \ldots \ldots \ldots \ldots$ & Sz 48SW & 873 & 2.06 & -5.50 & $0.0 \mathrm{E}+00$ & $\ldots$ & $\ldots$ & $\ldots$ & $\ldots$ \\
\hline $25 \ldots \ldots \ldots \ldots \ldots \ldots \ldots$ & Sz 50 & 117 & 0.07 & -1.93 & $0.0 \mathrm{E}+00$ & $\ldots$ & $\ldots$ & $\ldots$ & $\ldots$ \\
\hline $26 \ldots \ldots \ldots \ldots \ldots \ldots \ldots$ & WFI J13005531-7708295 & $\ldots$ & $\ldots$ & $\ldots$ & $\ldots$ & $\ldots$ & $\ldots$ & $\ldots$ & $\ldots$ \\
\hline $27 \ldots \ldots \ldots \ldots \ldots \ldots$ & RX J1301.0-7654a & $\ldots$ & $\ldots$ & $\ldots$ & $\ldots$ & $\ldots$ & $\ldots$ & $\ldots$ & $\ldots$ \\
\hline $28 \ldots \ldots \ldots \ldots \ldots \ldots$ & IRAS F12571-7657 & 73 & 0.09 & -3.91 & - & 300 & 0.36 & 0.72 & - \\
\hline $29 \ldots \ldots \ldots \ldots \ldots \ldots$ & Sz 51 & 28 & 0.20 & -2.61 & $0.0 \mathrm{E}+00$ & 100 & 0.14 & -0.84 & 1.E-07 \\
\hline $30 \ldots \ldots \ldots \ldots \ldots \ldots \ldots$ & CM Cha & 348 & 0.78 & -1.71 & $1.1 \mathrm{E}-07$ & 100 & 0.27 & 0.24 & 1.E-06 \\
\hline 31................... & $\mathrm{C} 50$ & 146 & 0.08 & -4.11 & $0.0 \mathrm{E}+00$ & $\cdots$ & $\cdots$ & $\cdots$ & $\cdots$ \\
\hline $32 \ldots \ldots \ldots \ldots \ldots \ldots \ldots$ & IRAS 12589-7646 & 405 & 0.47 & -6.87 & $0.0 \mathrm{E}+00$ & $\ldots$ & $\ldots$ & $\ldots$ & $\ldots$ \\
\hline $33 \ldots \ldots \ldots \ldots \ldots \ldots \ldots$ & RX J1303.1-7706 & $\ldots$ & $\cdots$ & $\ldots$ & $\cdots$ & $\cdots$ & $\cdots$ & $\ldots$ & $\cdots$ \\
\hline $34 \ldots \ldots \ldots \ldots \ldots \ldots \ldots$ & C51 & $\cdots$ & $\cdots$ & $\cdots$ & $\cdots$ & $\cdots$ & $\cdots$ & $\cdots$ & $\cdots$ \\
\hline $35 \ldots \ldots \ldots \ldots \ldots \ldots \ldots$ & WFI J13031615-7629381 & $\cdots$ & $\ldots$ & $\cdots$ & $\ldots$ & $\ldots$ & $\ldots$ & $\ldots$ & $\cdots$ \\
\hline $36 \ldots \ldots \ldots \ldots \ldots \ldots \ldots$ & Hn 22 & 68 & 1.18 & -4.29 & $0.0 \mathrm{E}+00$ & $\ldots$ & $\ldots$ & $\ldots$ & $\ldots$ \\
\hline $37 \ldots \ldots \ldots \ldots \ldots \ldots$ & Hn 23 & 293 & 0.18 & -3.57 & $0.0 \mathrm{E}+00$ & $\cdots$ & $\cdots$ & $\ldots$ & $\ldots$ \\
\hline $38 \ldots \ldots \ldots \ldots \ldots \ldots$ & Sz 52 & 177 & 0.35 & -2.34 & $0.0 \mathrm{E}+00$ & $\ldots$ & $\ldots$ & $\ldots$ & $\ldots$ \\
\hline 39...................... & Hn 24 & 58 & 0.06 & -4.27 & $0.0 \mathrm{E}+00$ & 300 & 0.05 & -2.15 & 1.E-09 \\
\hline $40 \ldots \ldots \ldots \ldots \ldots \ldots$ & Hn 25 & 118 & 0.19 & -2.30 & $3.8 \mathrm{E}-09$ & $\ldots$ & $\ldots$ & $\ldots$ & $\ldots$ \\
\hline $41 \ldots \ldots \ldots \ldots \ldots \ldots$ & Sz 53 & 118 & 0.03 & -2.30 & $3.8 \mathrm{E}-09$ & 100 & 0.14 & -0.84 & 1.E-07 \\
\hline $42 \ldots \ldots \ldots \ldots \ldots \ldots$ & Sz 54 & 93 & 0.09 & -1.84 & $2.4 \mathrm{E}-08$ & 100 & 0.06 & -1.49 & 1.E-08 \\
\hline $43 \ldots \ldots \ldots \ldots \ldots \ldots$ & SSTc2d J130521.7-773810 & 662 & 0.65 & -2.42 & $0.0 \mathrm{E}+00$ & 100 & 0.14 & -0.50 & 1.E-07 \\
\hline $44 \ldots \ldots \ldots \ldots \ldots \ldots \ldots$ & SSTc2d J130529.0-774140 & $\ldots$ & $\ldots$ & $\ldots$ & $\ldots$ & $\ldots$ & $\ldots$ & $\ldots$ & $\ldots$ \\
\hline $45 \ldots \ldots \ldots \ldots \ldots \ldots \ldots$ & SSTc2d J130540.8-773958 & 138 & 0.01 & -4.61 & $\ldots$ & $\ldots$ & $\ldots$ & $\ldots$ & $\ldots$ \\
\hline $46 \ldots \ldots \ldots \ldots \ldots \ldots \ldots$ & Sz 55 & 496 & 0.08 & -4.60 & $0.0 \mathrm{E}+00$ & 100 & 0.05 & -2.52 & 1.E-09 \\
\hline $47 \ldots \ldots \ldots \ldots \ldots \ldots$ & Sz 56 & 399 & 0.43 & -3.36 & $0.0 \mathrm{E}+00$ & $\ldots$ & $\ldots$ & $\ldots$ & $\ldots$ \\
\hline $48 \ldots \ldots \ldots \ldots \ldots \ldots$ & Sz 57 & 145 & 0.05 & -3.69 & $8.1 \mathrm{E}-09$ & $\ldots$ & $\ldots$ & $\ldots$ & $\ldots$ \\
\hline $49 \ldots \ldots \ldots \ldots \ldots \ldots$ & Sz 58 & 19 & 1.75 & -1.42 & $0.0 \mathrm{E}+00$ & 300 & 0.16 & -0.17 & 1.E-07 \\
\hline $50 \ldots \ldots \ldots \ldots \ldots \ldots$ & Sz 59 & 81 & 0.37 & -2.29 & $5.2 \mathrm{E}-08$ & 100 & 0.06 & -1.54 & 1.E-08 \\
\hline $51 \ldots \ldots \ldots \ldots \ldots \ldots$ & C62 & 134 & 0.02 & -4.32 & $\ldots$ & $\ldots$ & $\ldots$ & $\ldots$ & $\ldots$ \\
\hline $52 \ldots \ldots \ldots \ldots \ldots \ldots$ & $\mathrm{Sz} 60 \mathrm{~W}$ & 106 & 0.07 & -5.84 & $0.0 \mathrm{E}+00$ & 100 & 0.14 & -0.82 & 1.E-07 \\
\hline $53 \ldots \ldots \ldots \ldots \ldots \ldots \ldots$ & $\mathrm{Sz} 60 \mathrm{E}$ & 148 & 0.03 & -4.84 & $0.0 \mathrm{E}+00$ & $\ldots$ & $\ldots$ & $\ldots$ & $\ldots$ \\
\hline $54 \ldots \ldots \ldots \ldots \ldots \ldots$ & IRAS 13036-7644 & $\ldots$ & $\ldots$ & $\ldots$ & $\ldots$ & $\ldots$ & $\ldots$ & $\ldots$ & $\ldots$ \\
\hline $55 \ldots \ldots \ldots \ldots \ldots \ldots$ & Hn 26 & 568 & 0.23 & -2.30 & $0.0 \mathrm{E}+00$ & 100 & 0.14 & -0.84 & 1.E-07 \\
\hline $56 \ldots \ldots \ldots \ldots \ldots \ldots$ & Sz 61 & 674 & 0.66 & -2.35 & $4.5 \mathrm{E}-07$ & 100 & 0.36 & 0.00 & 1. $E-06$ \\
\hline $57 \ldots \ldots \ldots \ldots \ldots \ldots \ldots$ & C66 & 147 & 0.01 & -4.74 & $0.0 \mathrm{E}+00$ & $\ldots$ & $\ldots$ & $\ldots$ & $\ldots$ \\
\hline $58 \ldots \ldots \ldots \ldots \ldots \ldots \ldots$ & IRAS F13052-7653NW & 197 & 0.11 & -6.51 & $\ldots$ & $\ldots$ & $\ldots$ & $\ldots$ & $\ldots$ \\
\hline $59 \ldots \ldots \ldots \ldots \ldots \ldots$ & IRAS F13052-7653N & 498 & 3.54 & -5.53 & $\ldots$ & $\cdots$ & $\ldots$ & $\ldots$ & $\cdots$ \\
\hline $60 \ldots \ldots \ldots \ldots \ldots \ldots \ldots$ & Sz 62 & 150 & 0.05 & -3.83 & $4.1 \mathrm{E}-09$ & $\cdots$ & $\cdots$ & $\cdots$ & $\cdots$ \\
\hline 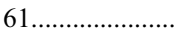 & Sz 63 & 81 & 3.02 & -3.17 & $0.0 \mathrm{E}+00$ & $\ldots$ & $\ldots$ & $\ldots$ & $\ldots$ \\
\hline $62 \ldots \ldots \ldots \ldots \ldots \ldots \ldots$ & 2MASS $13125238-7739182$ & 171 & 0.03 & -6.67 & $1.8 \mathrm{E}-08$ & $\ldots$ & $\ldots$ & $\cdots$ & $\cdots$ \\
\hline $63 \ldots \ldots \ldots \ldots \ldots \ldots \ldots$ & Sz 64 & 168 & 0.18 & -3.62 & $1.3 \mathrm{E}-07$ & $\ldots$ & $\ldots$ & $\ldots$ & $\ldots$ \\
\hline
\end{tabular}

${ }^{a}$ The mass accretion rate is given only for sources with $U$ and/or $B$ photometry (see $\S 5.6$ ). 

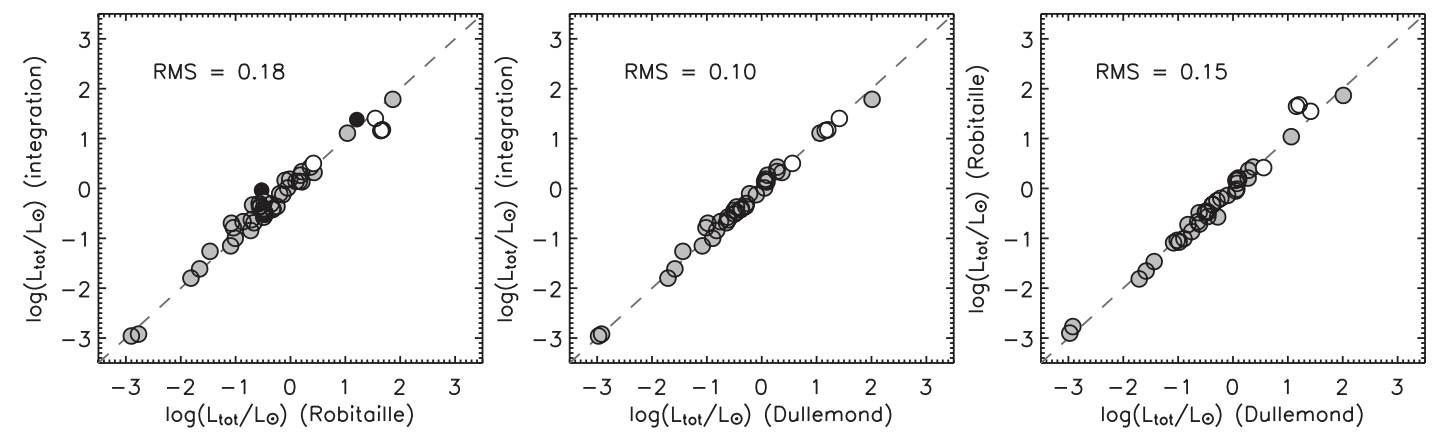

Fig. 13.-Comparison of the total luminosity derived with different methods as indicated in the axis labels. Symbols are as in Fig. 5. [See the electronic edition of the Journal for a color version of this figure.]

source IRAS 12500-7658: the SED is consistent with an embedded, very low mass object with $T_{\text {eff }}=2900 \mathrm{~K}$, mass accretion rate $\sim 2 \times 10^{-6} M_{\odot} \mathrm{yr}^{-1}$, and circumstellar dust parameters typical for very low mass forming objects. If the low temperature of this object will be spectroscopically confirmed, it will be an interesting case of a Class I source, very close to or below the substellar limit. It is worth noting that the object is detected in the $R$ and $I$ bands and that, using the techniques described in S07a, an effective temperature $T_{\text {eff }}=3000 \pm 200 \mathrm{~K}$ is independently estimated. However, the S07a methods may underestimate $T_{\text {eff }}$ if the extinction is high. The integrated luminosity, $L_{\text {bol }}=0.92 L_{\odot}$, is about 1 order of magnitude above the luminosity limit defining the so-called VeLLOs (very low luminosity objects; see, e.g., Dunham et al. 2006). Thus, another possibility is that what we are seeing in the optical is a very low mass companion, with the main luminosity source being invisible.

This section aims for a quantitative description of the circumstellar dust envelope properties found for the Cha II PMS population, which may provide some constraints for the disk evolution and planet formation models. However, since the disk/envelope parameters are strongly model dependent, we just compare the results among the different models in order to provide statistical ranges of these parameters and to quantify the deviations among different methods. Again, we caution that the output disk parameters are only the best-fit values and that a more careful analysis would require a number of model SEDs providing a good fit. This is, however, beyond the scope of this paper and is deferred to a future work.

An important output parameter from the models is the total object luminosity. We find a very good agreement between the total luminosities as derived in $\S 5.5$ and the total output luminosity from the D01 and R06 models: the rms difference relative to the former is within $0.1 \mathrm{dex}$ in $\log \left(L / L_{\odot}\right)$, while that relative to the latter is on the order of 0.18 dex (see Fig. 13); for some objects the total luminosity from the R06 fits is slightly lower (see Fig. 13), which is mainly a consequence of the fact that the R07 tool considers upper limits for the SED fits. The worse parameter sampling of the D'Alessio et al. (2005) models leads to some difficulties for a reasonable fit in some cases. The rms differences in luminosity relative to these models can be up to 0.5 dex. Differences in the total luminosity among models may also be due to the lack of data at short wavelengths in optical bands; for instance, the R06 fits tend to slightly overestimate the fluxes at wavelengths bluer than about $0.45 \mu \mathrm{m}$ relative to the D01 fits, when optical data are lacking. This happens in the SEDs of IRAS 12496-7650, Sz 49, and Hn 26 (see Fig. 7). Similarly, for Iso-Cha II 29 the R06 best-fit model flux is slightly underestimated relative to the SED data points at $\lambda<0.8 \mu \mathrm{m}$, while the D'Alessio et al. (2005) models are unable to reproduce its essentially photospheric points at around $10 \mu \mathrm{m}$.
A comparison of some of the output parameters resulting from the best fits of the D01 and R06 models is shown in the scatter plots in Figure 14, and a synthesis of the distribution of their values derived from each model is shown in the histograms in Figure 15 . There seems to be a fairly good correlation of the disk mass between the D01 and R06 models; however, the former models tend to overestimate the disk mass by about 1 order of magnitude relative to the latter. Geometrical parameters like the outer disk radius seem to be quite different among the different models; on the other hand, there seems to be a fair agreement of the inner disk radius for most objects, while for some the disk accretion models tend to overestimate this parameter relative to the reprocessing flared disk models. The difference is highest for Iso-Cha II 29, which is represented by the rightmost point in the bottom right panel of Figure 14. From a visual inspection of the fits, the most reliable disk parameters for this object seem to be those derived from the R06 models.

The discrepancies among the disk parameters derived from the different prescriptions might be in principle attributed to differences in the sampling of the parameter space covered by the models. This may be the case for the D'Alessio et al. (2005) models, but not for the R06 models that cover a wide range of disk parameters with a good sampling; also, the D01 tool calculates analytically the disk parameters based on the provided inputs. Thus, the differences on the disk parameters from the D01 and R06 models are most likely related to the fact that the two sets of models use different opacities and disk geometries, and that D01 considers flared reprocessing disks, while D'Alessio et al. (2005) and R06 reproduce the SEDs of accretion disks. Another possible source for the discrepancy among disk outer radii is linked to the lack of fluxes at millimeter wavelengths that allow stronger constraints on this parameter. The IRAC and MIPS wavelengths preferentially probe the inner parts of the disks; a good wavelength coverage should provide good measurements of the inner disk structures, differently with the outer disk radius, for which the mid-IR data are not sensitive. For the three models, the typical value for the disk inner radius is on the order of $0.1 \mathrm{AU}$ (see Fig. 15) with a similar range of this parameter among the different models, while the distribution of outer radius peaks at around $100 \mathrm{AU}$ for the accretion models but is smaller for the reprocessing models, again showing that this parameter is not well constrained because of the lack of millimeter data. Similarly, Figure 14 confirms that the inclination angle is mostly undetermined from the SED alone, a well-known result from previous works (e.g., D’Alessio et al. 1999; Chiang \& Goldreich 1999; R07 and references therein). We recall that differences may also be due to the fact that in our analysis the output disk parameters are only those resulting from the best-fit SED to each source. 

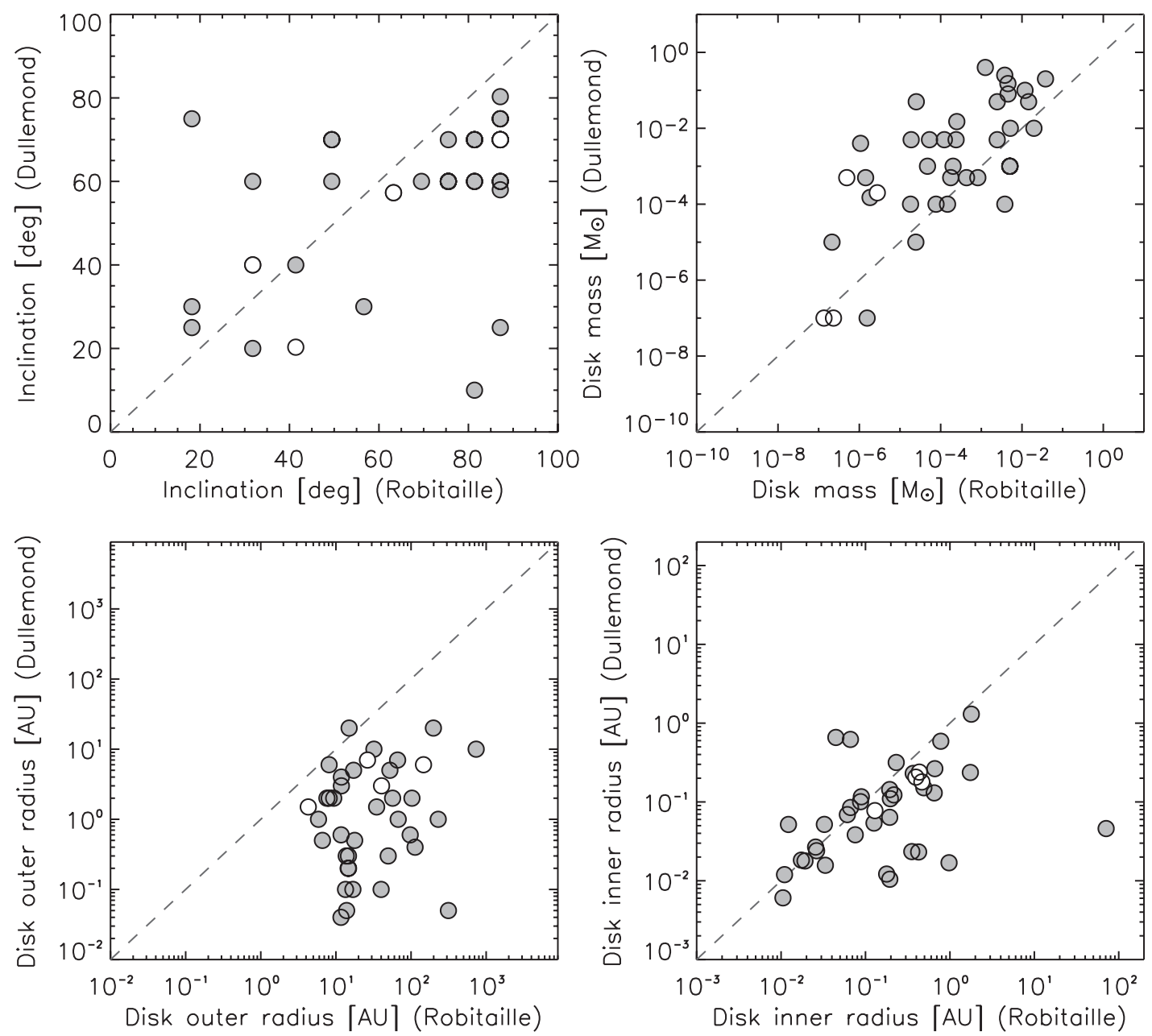

FIG. 14.-Comparison of some of the output parameters derived from the best fits to the SEDs using the D01 and R06 models as indicated in the axis labels. Symbols are as in Fig. 5. [See the electronic edition of the Journal for a color version of this figure.]
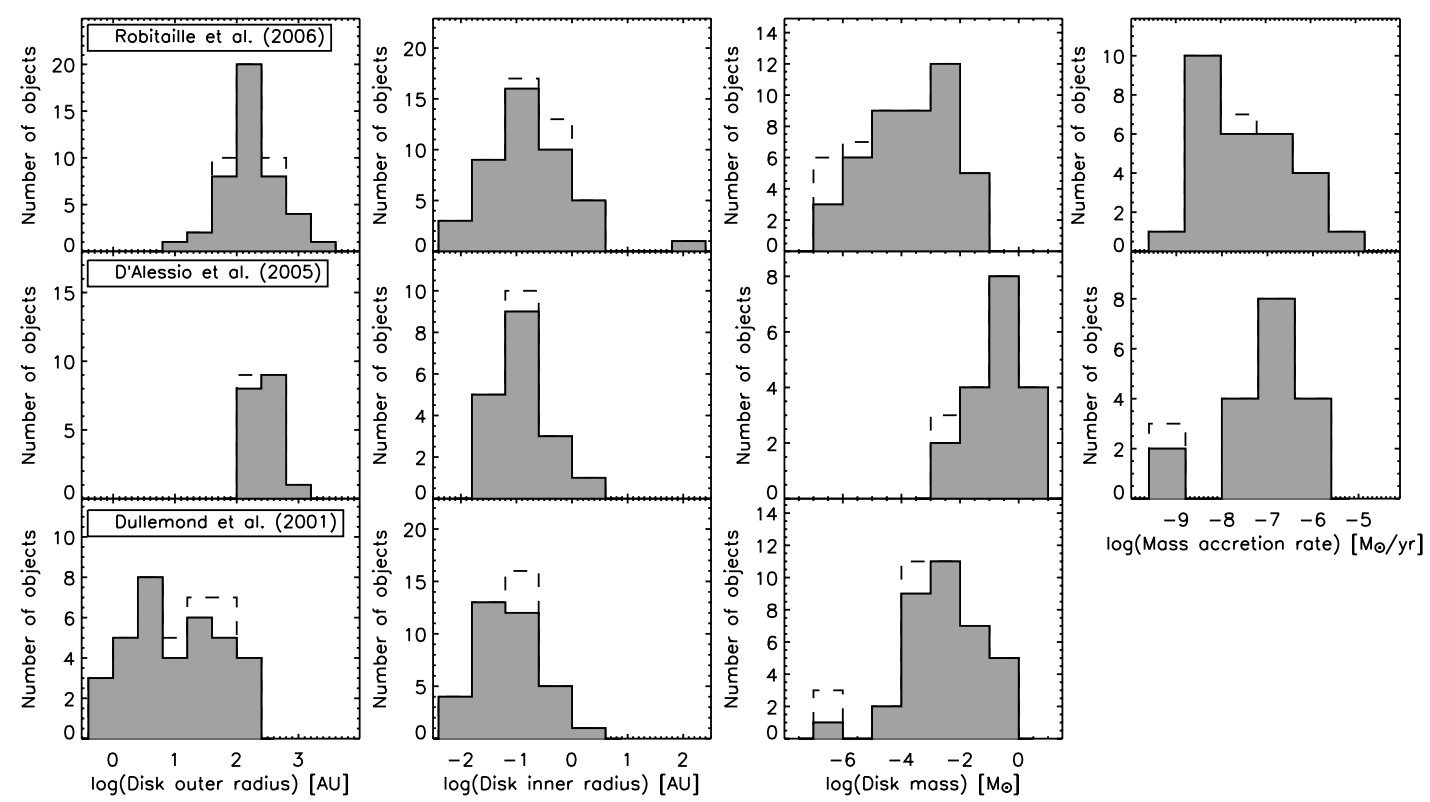

FIG. 15.-Distributions of relevant disk parameters resulting from the models as labeled in the leftmost panels. The solid histograms refer to the confirmed PMS objects, while the dashed ones represent the distributions when adding the candidate PMS objects. 
The disk mass for the sample ranges from about 1 to $10^{-4} M_{\odot}$ with the D01 models and from $10^{-2}$ to $10^{-6} M_{\odot}$ with the R06 models. The disk mass distribution from both models roughly peaks at around $10^{-3} M_{\odot}$. The D'Alessio et al. (2005) models provide higher disk masses than the D01 and R06 models.

Although the sample of objects for which we can provide the mass accretion rate is small, it is worthwhile to compare the value of this parameter as derived from the two accretion models. For some objects the accretion models agree within less than about 1 order of magnitude, but the statistics are small for any solid conclusion. The range of mass accretion rate determined from both accretion models is similar and goes from about $10^{-9}$ to about $10^{-6} M_{\odot} \mathrm{yr}^{-1}$.

\subsection{Comparison with Previous SED Fits}

An SED of IRAS 12496-7650 (or DK Cha) has been reported by Henning et al. (1993). These authors obtained $1.3 \mathrm{~mm}$ fluxes and performed the fit to the SED of the source by assuming both spherical and disk geometries. They found that the former model best fits the SED, except for the millimeter flux, while the disk model provides too much stellar flux in the NIR due to the assumption of a thin disk. Their disk model results for the disk radius $\left(R_{d} \approx 65 \mathrm{AU}\right)$ and disk inner radius $\left(R_{\mathrm{in}} \approx 0.13 \mathrm{AU}\right)$ are smaller than the values derived by both R06 $\left(R_{d} \approx\right.$ $\left.150 \mathrm{AU}, R_{\mathrm{in}} \approx 1.8 \mathrm{AU}\right)$ and D01 $\left(R_{d} \approx 200 \mathrm{AU}, R_{\mathrm{in}} \approx 1.3 \mathrm{AU}\right)$. The results of the disk mass are different among the different fits $\left[M_{d}\right.$ (Henning $) \approx 0.04 M_{\odot}, M_{d}($ Robitaille $) \approx 0.0013 M_{\odot}$, $M_{d}($ Dullemond $\left.) \approx 0.4 M_{\odot}\right]$. Using the $1.3 \mathrm{~mm}$ flux and equation (3) in Henning et al. (1993), a circumstellar mass of $0.07 M_{\odot}$ is derived.

A first fit of the SED of the Class I source Iso-Cha II 28 was performed by Persi et al. (2003) using the DUSTY code by Ivezic et al. (1999), which assumes an embedded source with a spherically symmetric dust envelope. The outer envelope radius they report is $\approx 1600 \mathrm{AU}$, while the value derived from the R06 fit is $\approx 2500 \mathrm{AU}$. The difference is most likely due to the assumption by Persi et al. (2003) of a spherically symmetric dust envelope, whereas the R06 model assumes a source with a disk and an envelope. Moreover, the envelope outer radius is not well constrained in the Persi et al. (2003) fit because they include only an upper limit for the (sub)millimeter flux. On the other hand, the envelope inner radius they fix $(\approx 0.16 \mathrm{AU})$ is much lower than the one derived from the R06 fit $(\approx 1 \mathrm{AU})$. Note also that the temperature of $4000 \mathrm{~K}$ assumed by Persi et al. (2003) for the central object is lower than the value derived from the R06 fit $(T \approx 4500 \mathrm{~K})$, but such a parameter is not constrained enough to say whether the difference of $500 \mathrm{~K}$ is really significant for such an embedded source. In conclusion, the differences on the resulting parameters come from the fact that the models are fundamentally different. This example only illustrates that fits to the SED of objects that are transitionary between envelope and disk stages are very degenerate, in particular when (sub)millimeter data are missing.

In addition, the SEDs of 10 classical T Tauri stars in Cha II were modeled by Cieza et al. (2005) using the D01 flared disk models. Their results on the disk inner radius are in very good agreement with those derived by us using the same models, except for the star Sz 62, for which Cieza et al. (2005) derived $R_{\text {in }} \approx 0.08 \mathrm{AU}$ and we obtain a value a factor of about 8 larger. We stress that the $70 \mu \mathrm{m}$ fluxes, which further constrain the SED fit, were not included in the SEDs by Cieza et al. (2005). When dropping the $70 \mu \mathrm{m}$ flux from the SED of Sz 62 , we obtain a closer value $\left(R_{\text {in }} \approx\right.$ $0.26 \mathrm{AU}$ ) to the one reported by Cieza et al. (2005) but still larger by a factor of about 3 .

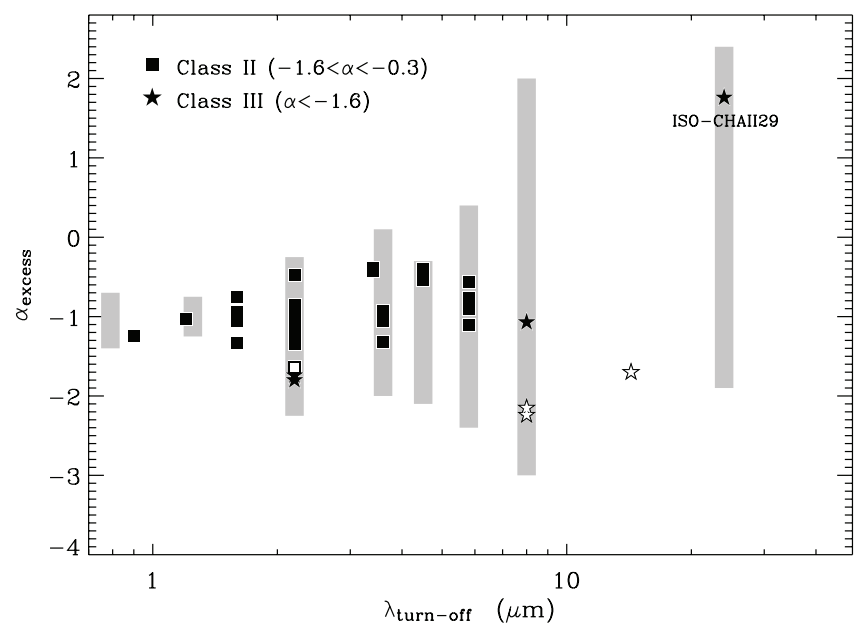

FIG. 16.-Distribution of excess slopes, $\alpha_{\text {excess }}$, with respect to the wavelength at which the IR excess begins, $\lambda_{\text {turnoff }}$, for the sample of confirmed ( filled symbols) and candidate (open symbols) PMS objects in Cha II. The symbols are as in Fig. 9. The intervals of $\alpha_{\text {excess }}$ for the classical and weak T Tauri stars were taken from Fig. 22 by $\mathrm{H} 07$ and are represented with shaded bars.

More recently, Allers et al. (2006) modeled the SEDs of six Cha II objects using the D01 models. The agreement between their results and ours using the same models is also quite good, except for SSTc2d J130540.8-773958 and C66, for which we get a slightly larger disk inner radius. We note also that the $70 \mu \mathrm{m}$ fluxes were not included in the SEDs by Allers et al. (2006), which might explain the small differences.

Finally, the SED of the substellar object Iso-Cha II 13 was modeled by Alcalá et al. (2006) also using the D01 flared disk models. The results are in agreement with those reported here for the D01 models.

\subsection{Disk Evolution Issues}

An interesting diagnostic to study disk evolution in young low-mass objects is the diagram of the excess slope, $\alpha_{\text {excess }}$, versus the wavelength at which the IR excess begins, $\lambda_{\text {turnoff }}$ (see Cieza et al. 2007 and references therein). The $\alpha_{\text {excess }}$ index is the SED slope determined for wavelengths equal to or longer than $\lambda_{\text {turnoff }}$. H07 compared the position of the Serpens YSOs in such a diagram with that of several classical and weak $\mathrm{T}$ Tauri stars and concluded that the Serpens objects follow the trend previously observed by Cieza et al. (2007), i.e., a wider range of excess slopes for later evolutionary phases. We have performed a similar analysis, which is shown in Figure 16. For the sake of clarity, the range of $\alpha_{\text {excess }}$ at each wavelength for the classical and weak T Tauri stars from Cieza et al. (2007) is represented here with shaded strips. The analysis is done only for the certified and candidate PMS objects with IR excess. Despite the low number statistics, the resulting $\alpha_{\text {excess }}$ and $\lambda_{\text {turnoff }}$ for the Cha II objects follow a similar trend as observed in Serpens by H07. The $\alpha_{\text {excess }}$ and $\lambda_{\text {turnoff }}$ values are reported in Table 8 .

The sources with a large $\alpha_{\text {excess }}$ and $\lambda_{\text {turnoff }}$, i.e., objects on the upper right of the diagram, are interpreted in terms of disks with large inner holes. One example in Cha II is Iso-Cha II 29. Its SED rises beyond $24 \mu \mathrm{m}$ (see Fig. 7). This object deserves further investigation with IR spectroscopy in order to assess possible signs of dust grain growth and settling to the disk midplane. This led us to investigate the relationship between the reference $\alpha$ index (i.e., $\alpha_{[K, \text { MIPS } 24 \mu \mathrm{m}]}$ ) and the disk inner radius. To this aim and considering the poor statistics, we divide the PMS objects and candidates in two samples according to $\lambda_{\text {turnoff }}$ in the following way: 
from the $\alpha_{\text {excess }}$ versus $\lambda_{\text {turnoff }}$ diagram we note that the dispersion of $\alpha_{\text {excess }}$ starts increasing at $\lambda_{\text {turnoff }} \sim 4-5 \mu \mathrm{m}$; under the assumption of thermal emission from a thin disk with blackbody grains, this corresponds to a distance of about $0.2 \mathrm{AU}$ from the central object. We thus use this limit to define two samples to investigate the dispersion of their $\alpha_{[K, \text { MIPS } 24 \mu \mathrm{m}]}$ index. We find that objects with disk inner radius larger than 0.2 AU show a dispersion of $\alpha_{[K, \text { MIPS } 24 \mu \mathrm{m}]}$ a factor of 2.5 larger than those with disk inner radius less than $0.2 \mathrm{AU}$. This may be interpreted in terms of larger inner disk radii for later evolutionary disk phases.

\subsection{Disk Fraction}

As concluded in $\S 5.3$, Lada classes in Cha II are quite consistent with their stages as defined by R06, and using $\alpha_{[K \text {, MIPS } 24 \mu \mathrm{m}]}=$ -1.6 is a good separation between thick and thin disks. Thus, sources with optically thick disks should have $\alpha_{[K, \text { MIPS } 24 \mu \mathrm{m}]}>$ -1.6 , while for anemic disks $-2.5<\alpha_{[K, \text { MIPS } 24 \mu \mathrm{m}]}<-1.6$. Objects with $\alpha_{[K, \text { MIPS } 24 \mu \mathrm{m}]}<-2.5$ should be characterized by normal late-type photospheres. From this classification the total fraction of objects with disks, either anemic or thick, would be on the order of $80 \%$, while those with thick disks would represent about $70 \%$ of the sample.

The disk fraction in Cha II is considerably higher than the values derived in other regions (i.e., in IC 348 by Lada et al. 2006) and would mean that if all the stars in Cha II were born with circumstellar disks, only a minor fraction of about $20 \%-30 \%$ have lost their primordial disks in about $4 \mathrm{Myr}$, the average age for the Cha II members (S07b). One may wonder whether our census might have missed a significant number of Class III objects, but this is unlikely. Although the $\mathrm{c} 2 \mathrm{~d}$ criteria select only IR excess objects, the other surveys are rather complete, in both space and flux. For instance, covering $1.75 \mathrm{deg}^{2}$, the survey by S07a picks up basically all low-mass objects $\left(M<1 M_{\odot}\right)$ without significant IR excess and is $95 \%$ complete down to a mass $M=0.03 M_{\odot}$, for a visual extinction $A_{V} \approx 2$ mag. In addition, our spatially unbiased spectroscopic survey with FLAMES (down to $R=18$ mag; $\mathrm{S} 07 \mathrm{~b}$ ) would have discovered at least some more Class III objects, if they existed. Furthermore, if many more Class III sources had been missed in optical and IR surveys, they would have been detected in X-rays, as they were in other regions, but only two (RX J1301.0-7654a and RX J1303.1-7706; Alcalá et al. 1995, 2000) were discovered. Although such X-ray surveys are not extremely deep, they would have detected many more Class III sources, if they were present. A further confirmation of this result comes from the negative detections by a deep XMM-Newton pointing in the northwest of the cloud (B. Stelzer 2007, private communication). Thus, unless all the Class III sources are located behind the cloud, which is very unlikely, the result on the high disk fraction in Cha II seems to be real.

The disk fraction as function of the central object mass in Cha II is shown in Figure 17. The average stellar mass, as derived by $\mathrm{S} 07 \mathrm{~b}$ for each individual object, was used for this analysis. In order to try to minimize the errors, we have divided the sample into four mass bins. ${ }^{19}$ Although the frequency of thick disks is apparently highest close to one solar mass, the low number statistics and hence the large errors prevent us from drawing any conclusion. However, this would be consistent with the results by Lada et al. (2006) that thick disks in IC 348 are more frequent

\footnotetext{
19 For this exercise we use the masses derived from the Baraffe et al. (1998) and Chabrier et al. (2000) tracks for stellar masses less than $1.4 M_{\odot}$, while for higher mass objects we use the masses derived from the Palla \& Stahler (1999) tracks, as the highest mass in the Baraffe et al. (1998) and Chabrier et al. (2000) tracks is $1.4 M_{\odot}$. These values are reported in $\mathrm{S} 07 \mathrm{~b}$.
}

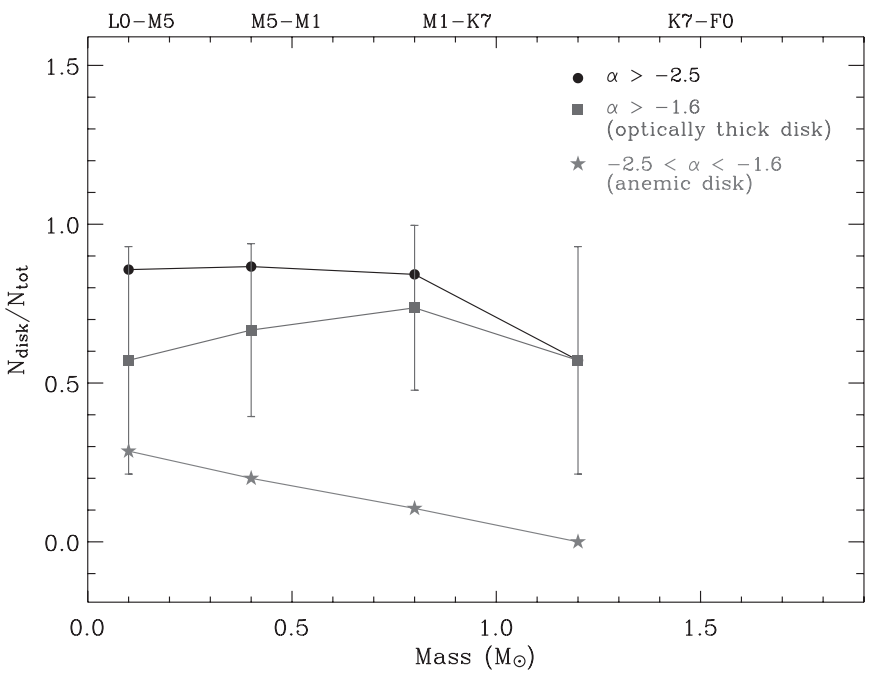

FIG. 17.-Disk fraction vs. mass of the central object. The symbols represent the different disk types, namely, anemic and thick, as indicated in the labels. The error bars, plotted only for the thick disks, represent the propagated Poissonian errors. The approximate spectral types corresponding to the four mass bins adopted are indicated at the top. [See the electronic edition of the Journal for a color version of this figure.]

for solar-mass stars. On the other hand, there seems to be a decline of anemic disks with stellar mass, but again the numbers are quite small.

It is also interesting to investigate the disk frequency in the substellar regime, but since only three out of the seven substellar objects (i.e., 43\%) are spectroscopically confirmed as members of Cha II (see S07b), the figures are not statistically significant; all three confirmed substellar objects possess optically thick disks according to the above definition, while the four substellar candidates posses anemic or no disks. Therefore, the disk fraction below the H-burning limit in Cha II strongly depends on the nature of these four candidates. If the latter will be all confirmed as members, the substellar disk fraction would be on the order of $40 \%$, i.e., similar to other regions; otherwise, it would be exceptionally high. We remind the reader that recent Spitzer studies in IC 348 by Luhman et al. (2005b) may indicate an increase of the disk fraction below the H-burning limit relative to that in stars (Lada et al. 2006).

\section{OVERALL RESULTS ON STAR FORMATION IN CHA II}

The physical parameters of the certified and candidate PMS objects are necessary for the study of the rate and efficiency of star formation of the cloud. We use here those determined by S07b.

\subsection{The Star Formation Rate}

One of the $\mathrm{c} 2 \mathrm{~d}$ goals is to compare the star formation rate (SFR) among the clouds of the Legacy survey. The results by $\mathrm{H} 07$ showed that the Serpens clouds turned some $60 M_{\odot}$ into YSOs every Myr. In order to homogeneously compare how much of the Cha II cloud mass is being transformed into YSOs, we have to use the total mass of the objects selected from the multicolor criteria by $\mathrm{H} 07$. The overall results of star formation in Cha II are summarized in Table 10. As mentioned in the previous sections, the number of YSOs in the $1.038 \mathrm{deg}^{2}$ IRAC 3.6-8 $\mu \mathrm{m} /$ MIPS $24 \mu \mathrm{m}$ overlap area in Cha II is 26 (see $\S 3$ and Table 1). Assuming a distance of $178 \mathrm{pc}$, the resulting IRAC 3.6-8 $\mu \mathrm{m} /$ MIPS $24 \mu \mathrm{m}$ overlap surveyed area in Cha II is about $10.02 \mathrm{pc}^{2}$. This means a density of about $2-3$ YSOs pc $^{-2}$, i.e., a factor of about 4-6 less 
TABLE 10

Numbers, Densities, and Star Formation Rates

\begin{tabular}{|c|c|c|c|c|c|c|c|c|}
\hline Type & $\begin{array}{c}\Omega \\
\left(\operatorname{deg}^{2}\right)\end{array}$ & $\begin{array}{l}\text { Area } \\
\left(\mathrm{pc}^{2}\right)\end{array}$ & $N$ & $\begin{array}{c}N / \Omega \\
\left(\operatorname{deg}^{-2}\right)\end{array}$ & $\begin{array}{c}\text { N/Area } \\
\left(\mathrm{pc}^{-2}\right)\end{array}$ & $\begin{array}{l}N / \mathrm{Vol}^{\mathrm{a}} \\
\left(\mathrm{pc}^{-3}\right)\end{array}$ & $\begin{array}{c}\mathrm{SFR} \\
\left(M_{\odot} \mathrm{Myr}^{-1}\right)\end{array}$ & $\begin{array}{c}\mathrm{SFR} / \text { Area } \\
\left(M_{\odot} \mathrm{Myr}^{-1} \mathrm{pc}^{-2}\right)\end{array}$ \\
\hline 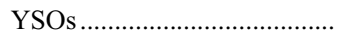 & 1.038 & 10.02 & 26 & 25 & 2.6 & 1.1 & 7.0 & 0.68 \\
\hline 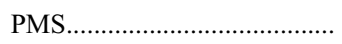 & 1.750 & 17.0 & 62 & 35 & 3.7 & 1.2 & 10.7 & 0.63 \\
\hline Cha II loose cluster................... & 0.944 & 9.11 & 48 & 51 & 5.3 & 2.3 & 12.5 & 0.90 \\
\hline A tight group & 0.022 & 0.211 & 6 & 275 & 28 & 82 & 1.6 & 9.5 \\
\hline 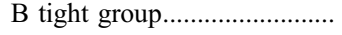 & 0.035 & 0.333 & 10 & 289 & 30 & 69 & 2.6 & 9.0 \\
\hline
\end{tabular}

${ }^{a}$ Volume estimated by assuming that the distribution of sources is locally spherical.

than in Serpens. The average mass of the Cha II members is $0.52 M_{\odot}(\mathrm{S} 07 \mathrm{~b})$, which means a total YSO mass of $\sim 14 M_{\odot}$. The typical age of these objects, determined from the D'Antona \& Mazzitelli (1997) and Palla \& Stahler (1999) theoretical isochrones, is on the order of $2 \mathrm{Myr}$ (S07b). This would mean that the Cha II cloud is turning some $7 M_{\odot}$ into YSOs every Myr, i.e., much less than the estimate in Serpens. A slightly higher star formation rate, but still less than in Serpens, is derived for the whole sample of certified and candidate PMS objects in the $1.75 \mathrm{deg}^{2}$ surveyed area, i.e., in $\sim 17 \mathrm{pc}^{2}$ : assuming the same average individual mass, we estimate a total mass of certified and candidate PMS objects on the order of $32 M_{\odot}$. Using the same theoretical isochrones, the resulting average age of the whole sample is on the order of $3 \mathrm{Myr}$ (S07b). Therefore, the star formation rate would be on the order of $10 M_{\odot} \mathrm{Myr}^{-1}$. The star formation rate in the other c2d clouds seems to be also higher than in Cha II (N. J. Evans et al. 2008, in preparation). It is worth noting that the star formation rate per unit area is quite similar for both PMS and YSO samples after correction for the larger area and older age of the PMS sample.

Using the results on clustering analysis in $\S 5.4$, we can also investigate the SFR for the substructures in Cha II. The results are summarized also in Table 10. The SFR per unit area and volume for the tight groups A and B, estimated by assuming an age of $2 \mathrm{Myr}$, is higher, but yet much lower than the values for the subgroups in Serpens and the other c2d clouds. On the other hand, the SFR of the Cha II loose cluster resembles the global SFR of the cloud determined above for the whole sample of PMS objects and candidates, as one should expect.

We therefore conclude that Cha II has the lowest SFR in comparison to the other four clouds investigated in the Legacy survey and hence it is currently the least active star-forming region among the $\mathrm{c} 2 \mathrm{~d}$ clouds.

\subsection{Cloud Mass and the Star Formation Efficiency}

To derive the star formation efficiency (SFE), we need an estimate of the cloud mass. Boulanger et al. (1998) combined CO observations with $I R A S$ images, H I observations, and extinction data to study the distribution of mass in the Chamaeleon cloud complex. The Cha II cloud has also been mapped in molecules such as ${ }^{12} \mathrm{CO}$ (Boulanger et al. 1998; Mizuno et al. 2001), ${ }^{13} \mathrm{CO}$ (Mizuno et al. 1998; Hayakawa et al. 2001), and $\mathrm{C}^{18} \mathrm{O}$ (Mizuno et al. 1999; Hayakawa et al. 2001). Estimates of the Cha II cloud mass based on these observations are reported in Table 11.

Another way to estimate the cloud mass is by means of extinction maps. Various combinations of maps in molecules, far-IR continuum, $\mathrm{H}$ I, extinction, and polarization toward field stars have been used to study the properties of the interstellar medium and magnetic field in the Chamaeleon clouds (Covino et al. 1997b). The $R_{V}$ ratio is found to be essentially normal (i.e., $\sim 3.1$ ) in the outer parts of the clouds, but several stars near the opaque core of Cha I show an unusually high value of $R_{V}$, which ranges between 5 and 6 , and there is some evidence that the Cha II cloud might show similar features, indicating that the two clouds are probably part of the same structure (Grasdalen et al. 1975; Rydgren 1980; Vrba \& Rydgren 1984; Steenman \& Thi 1989; Whittet et al. 1987, 1994, 1997; Luhman 2004). The extinction has been previously mapped in Cha II with a resolution of a few arcminutes by using IR star counts (Gregorio Hetem et al. 1988; Cambrésy 1999). The extinction has also been mapped using the c2d data. The c2d extinction maps were created at different spatial resolutions that range from $60^{\prime \prime}$ to $300^{\prime \prime}$. The details on how such maps were derived for each one of the five clouds of the $\mathrm{c} 2 \mathrm{~d}$ project can be found in the final data delivery document (Evans et al. 2007) and in H07. From the $\mathrm{c} 2 \mathrm{~d}$ map, the maximum extinction in the Cha II cloud is $A_{V} \approx 20 \mathrm{mag}$ and occurs close to IRAS 12496-7650 or DK Cha (see Fig. 9). The cloud mass as determined from the $\mathrm{c} 2 \mathrm{~d}$ extinction map is reported in Table 11. In addition, following the prescription by Kainulainen et al. (2006), we also calculated the cloud mass using the extinction maps by Cambrésy (1999) and J. Kainulainen (2006, private communication). There is a remarkable agreement of the mass estimates from the extinction maps and the $100 \mu \mathrm{m}$ emission.

Based on these mass estimates, we derived the global SFE in Cha II as SFE $=M_{\text {stars }} /\left(M_{\text {cloud }}+M_{\text {stars }}\right)$, where $M_{\text {cloud }}$ is the cloud mass and $M_{\text {star }}$ is the total mass in PMS objects or YSOs, which we estimate to be on the order of $32 M_{\odot}$ for the former and about $14 M_{\odot}$ for the latter (see $\S 6.1$ ). Note that this is the SFE so far as further star formation is possible. There is also the caveat that some of the gas that was used for the formation of some of the more evolved PMS objects has already been dispersed. The results on the

TABLE 11

Estimates of the Cloud Mass and SFE in Cha II

\begin{tabular}{|c|c|c|c|c|}
\hline \multirow[b]{2}{*}{ TRACER } & \multirow{2}{*}{$\begin{array}{l}\text { MASS } \\
\left(M_{\odot}\right)\end{array}$} & \multirow[b]{2}{*}{ REFERENCES } & \multicolumn{2}{|c|}{ SFE $(\%)$} \\
\hline & & & YSOs & PMS \\
\hline $100 \mu \mathrm{m} .$. & $900^{\mathrm{a}}$ & Boulanger et al. (1998) & 1.5 & 3.4 \\
\hline \multirow[t]{2}{*}{${ }^{12} \mathrm{CO} \ldots \ldots \ldots \ldots \ldots \ldots$} & $1250^{\mathrm{b}}$ & Boulanger et al. (1998) & 1.1 & 2.5 \\
\hline & $1860^{\mathrm{c}}$ & Mizuno et al. (2001) & 0.7 & 1.7 \\
\hline${ }^{13} \mathrm{CO} \ldots \ldots \ldots \ldots \ldots \ldots$ & $960^{\mathrm{c}}$ & Mizuno et al. (1999) & 1.4 & 3.2 \\
\hline \multirow[t]{3}{*}{ Extinction ............. } & $670^{\mathrm{d}}$ & This work & 2.0 & 4.6 \\
\hline & $830^{\mathrm{e}}$ & Cambrésy (1999) & 1.7 & 3.7 \\
\hline & $780^{\mathrm{e}}$ & $\begin{array}{l}\text { J. Kainulainen ( } 2006 \text {, } \\
\text { private communication) }\end{array}$ & 1.8 & 3.9 \\
\hline
\end{tabular}

\footnotetext{
a Total mass of $\mathrm{H}_{2}$ derived from the $100 \mu \mathrm{m}$ luminosity.

b Total mass of $\mathrm{H}_{2}$ derived from the ${ }^{12} \mathrm{CO}$ luminosity.

c Total mass of $\mathrm{H}_{2}$ derived from the ${ }^{13} \mathrm{CO}$ luminosity.

d Cloud mass for $A_{V}>2 \mathrm{mag}$.

e Cloud mass calculated using prescription by Kainulainen et al. (2006) for $A_{V}>2$ mag.
} 
TABLE 12

Estimates of the SFE for the Subgroups in Cha II

\begin{tabular}{ccccc}
\hline \hline Region & Number & $\begin{array}{c}M_{\text {subcloud }} \\
\left(M_{\odot}\right)\end{array}$ & $\begin{array}{c}M_{\text {objects }} \\
\left(M_{\odot}\right)\end{array}$ & SFE \\
\hline Cha II loose cluster.... & $48(22)$ & 400.7 & $25.0(11.4)$ & $5.8(2.8 \%)$ \\
A tight group .............. & $6(2)$ & 11.9 & $3.1(1.0)$ & $20.7(7.8 \%)$ \\
B tight group ............... & $10(6)$ & 14.7 & $5.2(3.1)$ & $26.1(17.4 \%)$ \\
Distributed...................... & $14(4)$ & 275.2 & $7.3(2.1)$ & $2.6(0.8 \%)$ \\
\hline
\end{tabular}

Notes. - The values for the c2d-identified YSOs are given in parentheses. $M_{\text {subcloud }}$ is the mass within volume density contour from $\mathrm{c} 2 \mathrm{~d}$ dust extinction maps. $M_{\text {objects }}$ is the total mass in objects estimated as $\left(0.52 M_{\odot}\right) N_{\text {objects }}$.

efficiency of star formation are provided in Table 11 . Consistently with the only previous estimate in literature $(\sim 1 \%$; Mizuno et al. 1999), we find that the SFE in Cha II ranges between $1 \%$ and $4 \%$ and that it is similar to the estimates for other T associations like Taurus (1\%-2\%; Mizuno et al. 1995) and Lupus (0.4-3.8\%; Tachihara et al. 1996), but surprisingly lower than the value derived for Cha I (13\%; Mizuno et al. 1999).

As suggested by Mizuno et al. (1999), the different star formation activities in the Chamaeleon clouds may reflect a different history of star formation, as a consequence of Cha I being older than Cha II. This hypothesis is not quite supported by our results, since the average age of the Cha I population is similar to that of Cha II (see results by S07b). The SFE estimates by Mizuno et al. (1999) assume an average stellar mass of 0.7 and $0.5 M_{\odot}$ for Cha I and Cha II, respectively, i.e., the average values determined by Alcalá et al. (1997). While for Cha II this assumption is consistent with the results by $\mathrm{S} 07 \mathrm{~b}$, i.e., $\sim 0.52 M_{\odot}$, the average value assumed for Cha I is significantly higher than measured by S07b, i.e., $\sim 0.45 M_{\odot}$, based on the more recent census of the Cha I population by Luhman (2004). Our estimate of the SFE for Cha I is on the order of $7.5 \%$, i.e., lower than the value derived by Mizuno et al. (1999) but yet significantly higher than our estimate for Cha II.

On the other hand, as mentioned in $\S 5.4$, Lepiné \& Duvert (1994) proposed arguments in favor of a common explanation for the displacement of the prominent nearby star formation regions with respect to the Galactic plane in terms of the impact of high-velocity clouds $\left(v \sim 100 \mathrm{~km} \mathrm{~s}^{-1}\right)$. This scenario would imply a common triggering agent for the star formation in Cha I and Cha II and hence a similar star formation activity, which is not supported by our SFE estimates.

At the level of the substructures, the SFE estimates are higher (see Table 12). Using the c2d extinction map, we calculated the mass enclosed within each structure as defined by the clustering analysis described in $\S 5.4$. By using the number of YSOs corresponding to each structure (see Table 7), we find that the efficiencies for the two tight $\mathrm{A}$ and $\mathrm{B}$ groups are as high as $20 \%-25 \%$, i.e., comparable to the efficiencies of dense clusters (e.g., LL03), but the SFE is only a few percent when averaged over the entire Cha II cluster and distributed group.

\section{CHA II OUTFLOWS}

In this section our data set is used to investigate the HH 52HH 54 outflow and its exciting source, as well as to search for other outflow candidates in Cha II.

\section{1. $H H$ 52, $H H$ 53, and HH 54}

The known outflow in Cha II is located at the northern edge of the cloud (see Spitzer images in Porras et al. [2007] and Fig. 18) and consists of several structures identified as the Herbig-Haro (HH) objects HH 52, HH 53, and HH 54 (Sandell et al. 1987).

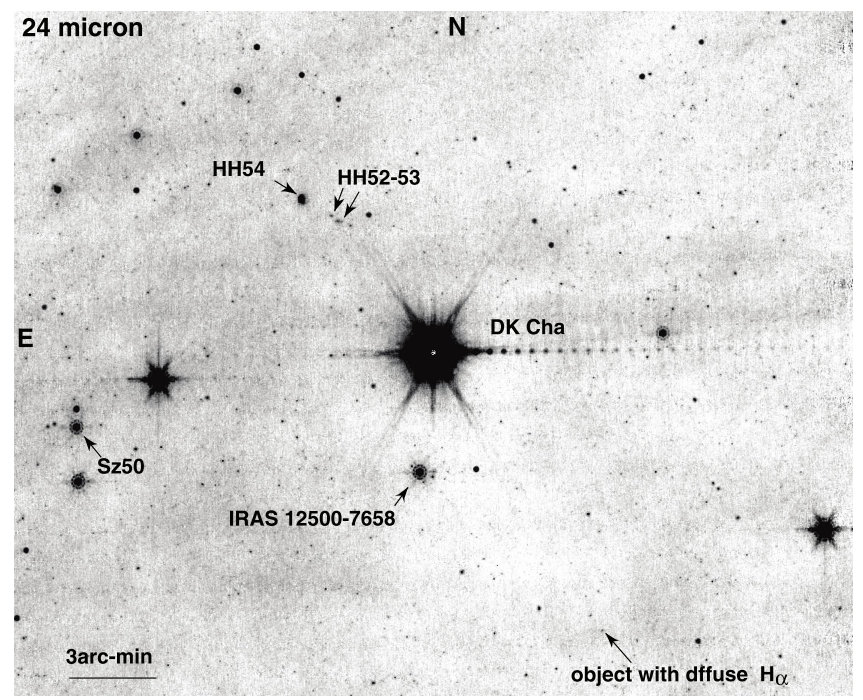

FIG. 18.- Part of the $24 \mu \mathrm{m}$ c2d mosaic in Cha II in gray-scale levels of intensity. Some of the sources discussed in the text are indicated. The object with diffused $\mathrm{H} \alpha$ emission discussed in $\S 7.2$ is also marked.

There has been controversy about the source driving the outflow: Sandell et al. (1987) proposed several possible sources that were later rejected in a spectroscopic study by Graham \& Hartigan (1988). However, the latter study revealed blueshifted gas with a radial velocity that becomes increasingly bluer along the stream from the northeast to the southwest. Based on these observations, Graham \& Hartigan (1988) proposed that IRAS 12496-7650, i.e., the embedded Herbig Ae star DK Cha located at about $14^{\prime}$ from $\mathrm{HH} 54$, might be the driving source of the outflow. Based on CO observations, Knee (1992) concluded that IRAS 124967650 is, however, unrelated to HH 52-HH 54, suggesting IRAS $12515-7641^{20}$ and IRAS $12522-7640$ as the driving sources of $\mathrm{HH}$ 52-HH 53 and HH 54, respectively.

We have investigated in all our images, from optical to $70 \mu \mathrm{m}$, both IRAS 12515-7641 and IRAS 12522-7640. The latter coincides with the head of the HH 54 bow shock (see Fig. 19) but in none of the images appears as a pointlike object. Note also that, as pointed out by Hughes et al. (1989), the source may have been mistakenly classified as a point source because of the line emission from the $\mathrm{HH}$ nebulosity itself. Moreover, its IRAS colors are incompatible with those of T Tauri stars or embedded objects (Prusti et al. 1992). Therefore, it is most likely produced by the IR emission of HH 54 itself. On the other hand, IRAS 125157641, or C30 (Vuong et al. 2001), appears as a pointlike object in all our images (see Fig. 19). This source was spectroscopically observed by us with FLAMES. Its optical spectrum is that of a late $\mathrm{K}$ type, most likely a background giant. Thus, contrary to the conclusions drawn from the CO study by Knee (1992), neither of these two IRAS sources seems to be the PMS star driving the outflow. Moreover, a proper-motion analysis of the infrared knots in HH 54 (Caratti o Garatti et al. 2006) led to the conclusion that its driving source should be located farther to the southwest.

If IRAS $12496-7650$ is not the driving source of the outflow, as concluded by Knee (1992), which source is then exciting HH 52-HH 54? Since our census for PMS objects in Cha II is rather complete, we inspected all our images to search for other possible candidates driving the outflow; we searched from the

20 This source, identified as IRAS C12515-7641 in SIMBAD and as IRAS F12514-7641 in Prusti et al. (1992), is also associated with the object C30 by Vuong et al. (2001). 

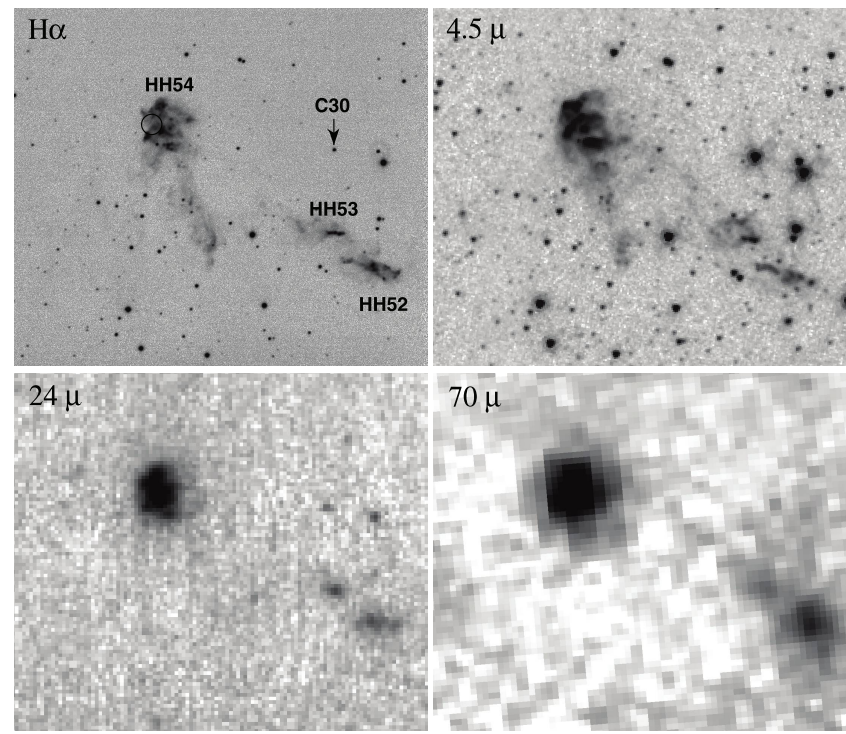

$70 \mu$

FIG. 19.-Images of the HH 52-HH 54 outflow at $\mathrm{H} \alpha$ (top left), $4.5 \mu \mathrm{m}$ (top right), $24 \mu \mathrm{m}$ (bottom left), and $70 \mu \mathrm{m}$ (bottom right). The HH objects and IRAS $12515-7641$ (or C30) are indicated in the WFI $\mathrm{H} \alpha$ image. The circle in this image marks the position of IRAS $12522-7640$. The images cover an area of approximately $4.5 \times 4 \mathrm{arcmin}^{2}$; north is up and east to the left.

northeast to the southwest direction of the stream of $\mathrm{HH} 52-$ HH 54, as suggested by the proper-motion study by Caratti o Garatti et al. (2006).

Figure 18 shows part of the $\mathrm{c} 2 \mathrm{~d}$ mosaic at $24 \mu \mathrm{m}$ with some interesting objects indicated. Besides IRAS 12496-7650, other relatively nearby candidates are C33 and WFI J12533662-7706393, located at about $2.3^{\prime}$ and $6.5^{\prime}$, respectively; these are, however, Class III candidate PMS objects without significant IR excess or strong winds. Thus, they are not expected to drive any outflow. Apart from IRAS 12496-7650 (DK Cha), the "nearest" object with strong IR emission in that direction is IRAS 12500-7658, one of the Class I sources in Cha II; its angular distance from HH 54 is about $20^{\prime}$, i.e., $\sim 1 \mathrm{pc}$ at the distance of Cha II, and its mass accretion rate, determined from the SED fit, is on the order of $2 \times 10^{-6} M_{\odot} \mathrm{yr}^{-1}$, i.e., typical for low-mass PMS stars driving outflows. We remind the reader that IRAS 12500-7658 might be a very low mass object (see $\S 5.6$ and results by S07b) and that the velocity of a jet is expected to correlate with the depth of the potential well; thus, in principle, one would expect that such a parsec-scale outflow is driven by a more massive star. However, no correlation exists between the mass of the driving source and the size of the outflows (for a review see Reipurth \& Bally 2001). We have thus searched for other possible $\mathrm{HH}$ features further to the southwest of IRAS $12500-7658$, but none were found. Nevertheless, an object with diffuse $\mathrm{H} \alpha$ emission (see $\S$ 7.2) located some $20^{\prime}$ to the southwest of DK Cha (see Fig. 18) was discovered, which would still favor this star as the driving source of the outflow. This is further discussed in $\S 7.2$.

Noteworthy, HH 54 is very bright at $24 \mu \mathrm{m}$. This can be seen in Figure 18 and in its zoomed version in Figure 19, where a comparison of the $\mathrm{H} \alpha, 4.5 \mu \mathrm{m}, 24 \mu \mathrm{m}$, and $70 \mu \mathrm{m}$ images is shown. Most of the $24 \mu \mathrm{m}$ emission comes from the [ $\left.\mathrm{S}_{\mathrm{I}}\right]$ and $[\mathrm{Fe}$ II] lines, as shown in the Spitzer spectrum of the object by Neufeld et al. (2006). As can be seen from Figure 19, the object is also well detected at $70 \mu \mathrm{m}$. We determined that HH 54 has a peak surface brightness of about $24 \mathrm{MJy} \mathrm{sr}^{-1}$ above the $70 \mu \mathrm{m}$ background. The total $70 \mu \mathrm{m}$ flux density is $0.68 \mathrm{Jy}$ in an arcmin square aperture, which is comparable to the $60 \mu \mathrm{m}$ flux density of IRAS 12522-7640 (see Liseau et al. 1996). If this flux density all came

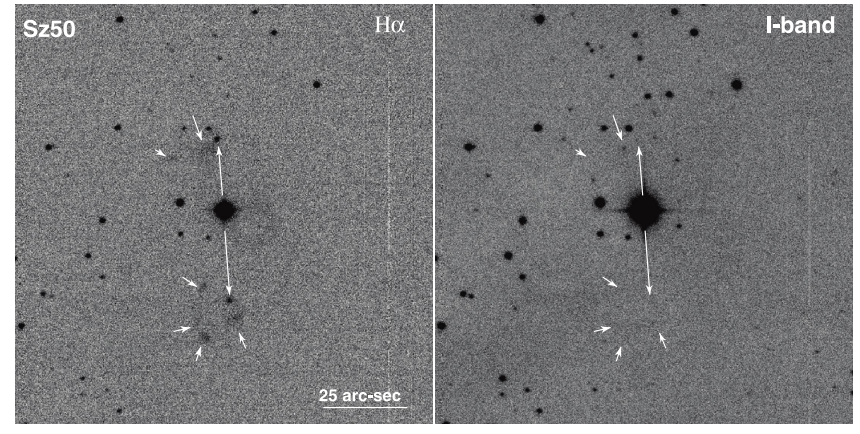

FIG. 20.- Images of the region around the classical T Tauri star $\mathrm{Sz} 50$ in $\mathrm{H} \alpha$ (left) and in the $I$ band (right). Sz 50 is in the center of the images. The arrows indicate the knots of $\mathrm{HH} 939$ and the diffuse emission in $\mathrm{H} \alpha$. North is up and east to the left. Both images cover an area of about $2 \times 2 \operatorname{arcmin}^{2}$.

from the [O I] line, the line flux would be on the order of $1.1 \times$ $10^{-11} \mathrm{ergs} \mathrm{s}^{-1} \mathrm{~cm}^{-2}$, i.e., close to, but slightly lower than, the value derived by Nisini et al. (1996) based on long-wavelength spectrometer (LWS) ISO data. Under the assumption of dissociative J-shocks, the [O I] luminosity is a very good diagnostic for mass-loss rate (Hollenbach 1985; Ceccarelli et al. 1997). Assuming the distance of $178 \mathrm{pc}$ and according to Hollenbach (1985), the mass-loss rate determined from the above [O I] line flux would be on the order of $1.1 \times 10^{-6} M_{\odot} \mathrm{yr}^{-1}$, which is a factor of about 2 less than derived by Nisini et al. (1996), but the difference is mainly due to the larger distance $(250 \mathrm{pc})$ these authors assume for Cha II. Based on measurements of the [O I] line flux, Nisini et al. (1996) derived values of the mass-loss rate for HH 52 and HH 53, which are similar between each other, but lower than the one derived for HH 54. This suggests, as these authors conclude, that the wind source driving $\mathrm{HH} 54$ should be other than the one driving $\mathrm{HH} 52$ and $\mathrm{HH}$ 53. The mass-loss rate they measure for IRAS 12496-7650 (DK Cha), also from an LWS spectrum, is very similar to the one they derived for HH 52 and HH 53 . Therefore, they cannot exclude that the driving source for at least the latter two is precisely IRAS $12496-7650$. The mass-loss rate of the order of $10^{-6} M_{\odot} \mathrm{yr}^{-1}$ would place the three $\mathrm{HH}$ objects among the most powerful known, and according to the measurements by Hartigan et al. (1995), a driving source with an accretion rate on the order of a few times $10^{-5} M_{\odot} \mathrm{yr}^{-1}$ would be necessary to produce such a mass loss. The accretion rate of the Class I source IRAS $12500-7658$ would be probably high enough. Thus, a scenario in which IRAS $12500-7658$ is driving HH 54, while IRAS 12496-7650 drives HH 52 and HH 53, might be feasible. However, given the uncertainties involved, one cannot completely rule out that IRAS $12496-7650$, i.e., DK Cha, is the driving source of the three HH objects. The HH 52-HH 54 outflow is not associated with any of the tight groups A or B identified in $\S 5.4$, but both DK Cha and the outflow can be associated with the loose cluster.

\subsection{New Outflows}

By inspection of our images, and in particular those in $\mathrm{H} \alpha$, it was possible to identify other possible candidates to outflows in Cha II. These candidates are shown in Figures 20 and 21, where the WFI $\mathrm{H} \alpha$ narrowband images are compared with those in the $I$ band. In both cases, the diffuse $\mathrm{H} \alpha$ emission is not detected in the $I$ band, which demonstrates that the structures shown cannot be of extragalactic nature.

Two compact $\mathrm{H} \alpha$ emission knots are found on opposite sides of the classical T Tauri star Sz 50 (see Fig. 20). Both are roughly circular with FWHM $20 \%$ larger than nearby point sources, 


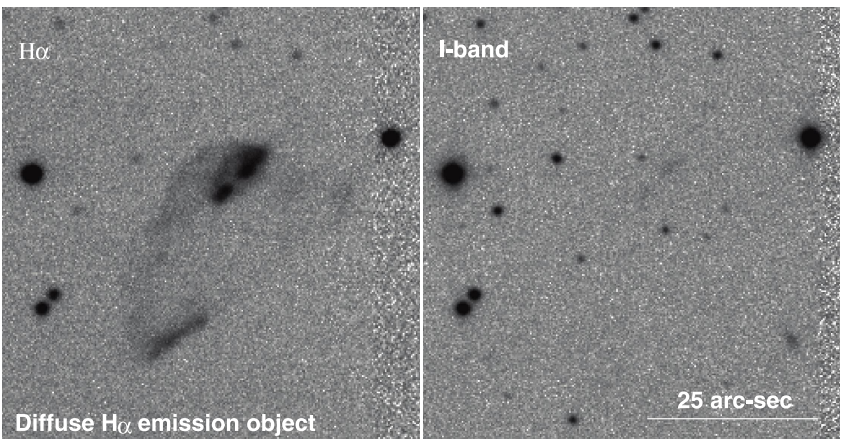

FIG. 21.- Images of the diffused $\mathrm{H} \alpha$ emission object in $\mathrm{H} \alpha$ (left $)$ and in the $I$ band (right). North is up and east to the left. Both images cover an area of about $1 \times 1 \operatorname{arcmin}^{2}$.

implying deconvolved source sizes of $0.5^{\prime \prime}-1^{\prime \prime}$. These two knots presumably trace a bipolar Herbig-Haro flow from $\mathrm{Sz} 50$, which is consistent with the detection of [O I] emission at $6300 \AA$ in the stellar spectrum by Hughes \& Hartigan (1992). No counterparts to these knots are detected in the Spitzer IRAC images, which are sensitive to rotational $\mathrm{H}_{2}$ emission; this suggests that the shocked material is primarily atomic. The flow axis follows P.A. $=4^{\circ}$, with one knot $21.2^{\prime \prime}$ north of the star and the other $26.7^{\prime \prime}$ to the south. Diffuse $\mathrm{H} \alpha$ emission is seen to the east of both knots, suggesting that deeper images might detect a greater flow extent. The bipolar flow spans a projected distance of $\sim 8500 \mathrm{AU}$. For a typical outflow velocity of $200 \mathrm{~km} \mathrm{~s}^{-1}$, the observed flow features would have an age of $\sim 100 \mathrm{yr}$. This object has been assigned the number $\mathrm{HH} 939$, following the criteria for the $\mathrm{HH}$ number designation (Reipurth 1994). The positions of the compact $\mathrm{H} \alpha$ emission knots of $\mathrm{HH} 939$ are reported in Table 13. The HH 939 outflow and its driving source fall right in the center of the tight group A identified in $\S 5.4$.

Another object with diffuse $\mathrm{H} \alpha$ emission has been found at some $20^{\prime}$ to the southwest of IRAS 12496-7650 (DK Cha). Images in $\mathrm{H} \alpha$ and in the $I$ band are shown in Figure 21. The object, also marked in Figure 18, is barely detected at $24 \mu \mathrm{m}$; unfortunately, we cannot verify possible $\mathrm{H}_{2}$ emission because the object falls outside the area covered with IRAC. While searching in all our images, we found no obvious driving source for this object. Note, however, that IRAS 12496-7650 (DK Cha) is located about halfway between HH 54 and this $\mathrm{H} \alpha$ object (see Fig. 18). Such geometry would suggest that it could be the counterpart of HH 52-HH 54, supporting the hypothesis that the embedded Herbig star is driving both $\mathrm{HH}$ features. However, assuming that the new one is indeed an HH object, the object's morphology (see Fig. 21) is not what one would expect for a flow coming from the northeast, casting some doubts that it may be part of the famous outflow. We extensively inspected all the images to search for other possible HH-like objects to the southwest of IRAS 12496-7650 (DK Cha), but no other interesting features were found. More data in IR bands and in CO are necessary to investigate in more depth the nature of the diffuse $\mathrm{H} \alpha$-emitting object and eventually confirm whether it is part of the HH 52-HH 54 outflow.

\section{SUMMARY}

Based on Spitzer c2d IRAC and MIPS observations, complemented with optical and NIR data, we have performed a census of the PMS population in Cha II. The properties of the PMS objects and candidates, as well as of their circumstellar matter, have been studied. The population consists of 51 certified and 11 candidate PMS objects in about $1.75 \mathrm{deg}^{2}$. The census is complete down to $M \sim 0.03 M_{\odot}$.
TABLE 13

Components of the HH 939 Outflow

\begin{tabular}{ccc}
\hline \hline HH & $\begin{array}{c}\text { R.A. } \\
\text { (J2000.0) }\end{array}$ & $\begin{array}{c}\text { Decl. } \\
\text { (J2000.0) }\end{array}$ \\
\hline HH 939 N .................... & 130055.92 & -771001.0 \\
HH 939 S................. & 130054.92 & -771049.0 \\
\hline
\end{tabular}

Note.- Units of right ascension are hours, minutes, and seconds, and units of declination are degrees, arcminutes, and arcseconds.

We have investigated both the YSOs with infrared excess selected according to the $\mathrm{c} 2 \mathrm{~d}$ criteria and the other PMS cloud members and candidate PMS objects from the previous surveys. The c2d multicolor criteria select 26 YSOs in the IRAC $3.6-8 \mu \mathrm{m} /$ MIPS $24 \mu \mathrm{m}$ overlapping area of $1.038 \mathrm{deg}^{2}$ in Cha II, where $A_{V}>2$; the new c2d selection criteria work very well in cleaning the samples of YSO candidates from background extragalactic contaminants. Most PMS objects and candidates off the IRAC $3.6-8 \mu \mathrm{m} /$ MIPS $24 \mu \mathrm{m}$ overlapping area show IR excess; thus, they would have been selected with such criteria if IRAC 3.6-8 $\mu \mathrm{m}$ /MIPS $24 \mu \mathrm{m}$ data had been available. Our spectroscopic follow-ups, published elsewhere, confirmed the PMS nature of most of the selected candidates, supporting the reliability of the new c2d selection criteria and of previous selections.

We studied the volume density of the 62 PMS objects and candidates with the following results: two tight groups, with volume densities higher than $25 M_{\odot} \mathrm{pc}^{-3}$, can be identified, but neither has enough members to qualify them as separate clusters. The spatial distribution of these groups is well correlated with the regions of high extinction as derived from the $\mathrm{c} 2 \mathrm{~d}$ extinction map. On the other hand, at $1 M_{\odot} \mathrm{pc}^{-3}$, corresponding to the level proposed by LL03 to define clusters, Cha II as a whole can be defined as a loose cluster. Most $(75 \%)$ of the sources in Cha II are associated with this cluster, which also includes both tight subgroups.

Seven of the PMS objects and candidates are visual multiples with separations $0.8^{\prime \prime}-6.0^{\prime \prime}$ and are thus blended in the IRAC or MIPS images. This gives a fraction of the order of $13 \% \pm 3 \%$ in the separation range of 140-1060 $\mathrm{AU}$, which is consistent with the observations in PMS clusters. We did not find significant variability, on a timescale of $6 \mathrm{hr}$, in any of the Cha II certified or candidate PMS objects, in line with other $\mathrm{c} 2 \mathrm{~d}$ investigations.

The analysis of the colors and SEDs shows that the Cha II population is dominated by objects with active accretion, with only a minority being systems with passive disks. The average PMS object in Cha II is the one for which the disk luminosity is about one-third of the stellar luminosity. Most objects in Cha II possess typical SEDs corresponding to disks that evolve more or less homogeneously from flared to flat. The disk fraction in Cha II of $70 \%-80 \%$ is exceptionally high in comparison with other star formation regions. If all the stars in Cha II were born with circumstellar disks, only a minor fraction of about $20 \%-30 \%$ have lost their disks in about 2-3 Myr, i.e., the typical age of Cha II members. Such high disk fraction and the very small number of Class I sources relative to Class II sources may indicate that star formation in Chamaeleon has occurred rapidly a few million years ago.

Many of the PMS stars close to the H-burning limit and all the legitimate substellar objects possess optically thick disks, confirming that disks are common down to the very low mass regime, in agreement with previous findings in other regions of star formation. A trend for the optically thick disks to be more frequent in 
solar-mass stars is observed, but it is blurred by the poor statistics in Cha II. No evidence is found that more luminous stars, being more likely multiples, possess less massive disks with big holes as a consequence of rapid disk dissipation due to multiplicity.

The cloud mass determined from the extinction maps is on the order of $1000 M_{\odot}$, and the SFE of $1 \%-4 \%$ is similar to our estimates for other T associations like Taurus and Lupus, but significantly lower than for Cha I $(\sim 7 \%)$. This suggests that different star formation activities in the Chamaeleon clouds may reflect a different history of star formation. The Cha II cloud turned about $7 M_{\odot}$ into stars every Myr, i.e., less than the star formation rate in other star-forming regions. At the level of the tight groups, the SFR and SFE are much higher, but yet lower than in the other $\mathrm{c} 2 \mathrm{~d}$ clouds.

Finally, an extensive investigation on the controversial driving source of the HH 52-HH 54 outflow has been performed. Our data set seems to still favor DK Cha as the driving source, but large-scale sensitive observations in $\mathrm{CO}$ are necessary to firmly establish this issue. The HH 52-HH 54 outflow is very well detected at $70 \mu \mathrm{m}$ with a flux density comparable to that of IRAS $12522-7640$ at $60 \mu \mathrm{m}$. If this flux density all came from the [O I] line, the line flux would be on the order of $1.1 \times 10^{-11} \mathrm{ergs} \mathrm{s}^{-1}$ $\mathrm{cm}^{-2}$. A new Herbig-Haro outflow, HH 939, has been discovered based on our WFI $\mathrm{H} \alpha$ images. The driving source of the $\mathrm{HH}$ knots is the classical T Tauri star Sz 50.

We thank the anonymous referee for his careful reading and useful comments/suggestions. Support for this work, part of the
Spitzer Legacy Science Program, was provided by NASA through contract 1224608 issued by the Jet Propulsion Laboratory, California Institute of Technology, under NASA contract 1407. This work was partially financed by the Istituto Nazionale di Astrofisica (INAF) through PRIN-INAF-2005. L. S. acknowledges financial support from PRIN-INAF-2005 (Stellar clusters: a benchmark for star formation and stellar evolution), and B. M. thanks the Fundación Ramón Areces for financial support. We thank T. Robitaille for discussions and help on the use of his accretion models and J. Kainulainen for providing an unpublished extinction map of Cha II and for discussions on extinction issues. We are also grateful to Ewine van Dishoeck for several discussions, comments, and suggestions on an earlier version of the paper and to Paul Harvey for the many discussions on the YSO selection and for his comments on the paper. We also thank Bo Reipurth for discussions on $\mathrm{HH}$ objects and B. Stelzer for communications on unpublished results of XMM-Newton observations in Cha II. We also thank Tyler Bourke for his comments. A special thanks to pussycat Matula for her assiduous and warm assistantship during the preparation of this manuscript. We acknowledge the c $2 \mathrm{~d}$ collaborators for the many discussions and suggestions during the telecons. This paper is based on observations carried out at the European Southern Observatory, La Silla and Paranal (Chile), under proposals 67.C-0225, 68.C-0311, 076.C-0385, and 078.C-0293. This publication makes use of data products from the Two Micron All Sky Survey, which is a joint project of the University of Massachusetts and the Infrared Processing and Analysis Center/ California Institute of Technology, funded by NASA and the National Science Foundation. We also acknowledge extensive use of the SIMBAD database.
Adams, F. C., Lada, C. J., \& Shu, F. H. 1987, ApJ, 312, 788

Alcalá, J. M., et al. 1995, A\&AS, 114, 109

1997, A\&A, 319, 184

2000, A\&A, 355, 629

2006, A\&A, 453, L1

Allard, F., \& Hauschildt, P. H. 1995, ApJ, 445, 433

Allard, F., Hauschildt, P. H., \& Schwenke, D. 2000, ApJ, 540, 1005

Allers, K. N. 2006, Ph.D. thesis, Univ. Texas at Austin

Allers, K. N., Kessler-Silacci, J. E., Cieza, L. A., \& Jaffe, D. T. 2006, ApJ, 644, 364

Allers, K. N., et al. 2007, ApJ, 657, 511

Backman, D. E., \& Paresce, F. 1993, in Protostars and Planets III, ed. E. H. Levy \& J. I. Lunine (Tucson: Univ. Arizona Press), 1253

Baraffe, I., Chabrier, G., Allard, F., \& Hauschildt, P. H. 1998, A\&A, 337, 403

Barrado y Navascués, D., \& Jayawardhana, R. 2004, ApJ, 615, 840

Beichmann, C. A., Helou, G., \& Walker, D. W. 1988, IRAS Point Source Catalog (NASA RP-1190; Washington, DC: GPO)

Bertin, E., \& Arnouts, S. 1996, A\&AS, 117, 393

Boulanger, F., Bronfman, L., Dame, T. M., \& Thaddeus, P. 1998, A\&A, 332, 273

Bourke, T. L., Hyland, A. R., \& Robinson, G. 1995, MNRAS, 276, 1052

Brandner, W., \& Zinnecker, H. 1997, A\&A, 321, 220

Calvet, N., et al. 2005, ApJ, 630, L185

Cambrésy, L. 1999, A\&A, 345, 965

Caratti o Garatti, A., Giannini, T., Nisini, B., \& Lorenzetti, D. 2006, A\&A, 449, 1077

Ceccarelli, C., Haas, M. R., Hollenbach, D. J., \& Rudolph, A. L. 1997, ApJ, 476, 771

Chabrier, G., Baraffe, I., Allard, F., \& Hauschildt, P. 2000, ApJ, 542, 464

Chiang, E. I., \& Goldreich, P. 1999, ApJ, 519, 279

Cieza, L., et al. 2005, ApJ, 635, 422 2007, ApJ, 667, 308

Cohen, M. 1973, MNRAS, 164, 395

Covino, E., et al. 1997a, A\&A, 328, 187 1997b, A\&AS, 122, 95

Cutri, R. M., et al. 2003, The IRSA 2MASS Point Source Catalog, NASA/IPAC Infrared Science Archive

D’Alessio, P., et al. 1999, ApJ, 527, 893 2005, Rev. Mex. AA, 41, 61

\section{REFERENCES}

D’Antona, F., \& Mazzitelli, I. 1997, Mem. Soc. Astron. Italiana, 68, 807

Ducourant, C., et al. 2005, A\&A, 438, 769

Dullemond, C. P., Dominik, C., \& Natta, A. 2001, ApJ, 560, 957 (D01)

Dullemond, C. P., Hollenbach, D., Kamp, I., \& D'Alessio, P. 2007, in Protostars and Planets V, ed. B. Reipurth, D. Jewitt, \& K. Keil (Tucson: Univ. Arizona Press), 555

Dunham, M. M., et al. 2006, ApJ, 651, 945

Evans, N. J., II, Harvey, P., \& Dunham, M. M. 2005, Third Delivery of Data from the c2d Legacy Project: IRAC and MIPS (Pasadena: SSC)

. 2007, Final Delivery of Data from the c2d Legacy Project: IRAC and MIPS (Pasadena: SSC)

Evans, N. J., II, et al. 2003, PASP, 115, 965

Gauvin, L. S., \& Strom, K. M. 1992, ApJ, 385, 217

Graham, J. A., \& Hartigan, P. 1988, AJ, 95, 1197

Grasdalen, G., et al. 1975, AJ, 80, 117

Greene, T. P., et al. 1994, ApJ, 434, 614

Gregorio Hetem, J. C., Sanzovo, G. C., \& Lepine, J. R. D. 1988, A\&AS, 76, 347

Gutermuth, R. A., et al. 2005, ApJ, 632, 397

Hartigan, P. 1993, AJ, 105, 1511

Hartigan, P., Edwards, S., \& Ghandour, L. 1995, ApJ, 452, 736

Hartmann, L., et al. 2005, ApJ, 629, 881

Harvey, P., et al. 2006, ApJ, 644, 307 2007, ApJ, 663, 1149 (H07)

Hauschildt, P. H., Allard, F., \& Baron, E. 1999, ApJ, 512, 377

Hayakawa, T., et al. 2001, PASJ, 53, 1109

Henning, T., Pfau, W., Zinnecker, H., \& Prusti, T. 1993, A\&A, 276, 129

Hollenbach, D. J. 1985, Icarus, 61, 36

Hughes, J. H., Emerson, J. P., Zinnecker, H., \& Whitelock, P. A. 1989, MNRAS, 236, 117

Hughes, J. H., \& Hartigan, P. 1992, AJ, 104, 680

Ivezic, Z., Nenkova, M., \& Elitzur, M. 1999, User Manual for Dusty (Univ. Kentucky), Internal Rep.

Jayawardhana, R., \& Ivanov, V. D. 2006, ApJ, 647, L167

Jørgensen, J. K., Johnstone, D., van Dishoeck, E. F., \& Doty, S. D. 2006, A\&A, 449, 609

Kainulainen, J., Lehtinen, J., \& Harju, J. 2006, A\&A, 447, 597

Kenyon, S., \& Hartmann, L. 1987, ApJ, 323, 714

Knee, L. B. G. 1992, A\&A, 259, 283 
Köhler, R. 2001, AJ, 122, 3325

Lada, C. J. 1987, in IAU Symp. 115, Star Forming Regions, ed. M. Peimbert \& J. Jugaku (Dordrecht: Reidel), 1

Lada, C. J., \& Lada, E. A. 2003, ARA\&A, 41, 57 (LL03)

Lada, C. J., et al. 2006, AJ, 131, 1574

Lagrange, A. M., Backman, D. E., \& Artymowicz, P. 2000, in Protostars and Planets IV, ed. V. Mannings, A. P. Boss, \& S. S. Russell (Tucson: Univ. Arizona Press), 639

Larson, K. A., Whittet, D. C. B., Prusti, T., \& Chiar, J. E. 1998, A\&A, 337, 465

Lehtinen, K., Mattila, K., \& Lemke, D. 2005, A\&A, 437, 159

Lepiné, J., \& Duvert, G. 1994, A\&A, 286, 60

Liseau, R., et al. 1996, A\&A, 315, L181

Luhman, K. L. 2004, ApJ, 602, 816

Luhman, K. L., et al. 2005a, ApJ, 620, L51

2005b, ApJ, 631, L69

2005c, ApJ, 635, L93

Lynden-Bell, D., \& Pringle, J. E. 1974, MNRAS, 168, 603

Mizuno, A., et al. 1995, ApJ, 445, L161

1998, ApJ, 507, L83

1999, PASJ, 51, 859
2001, PASJ, 53, 1071

Neufeld, D. A., Melnick, G. J., \& Harwit, M. 1998, ApJ, 506, L75

Neufeld, D. A., et al. 2006, ApJ, 649, 816

Nisini, B., et al. 1996, A\&A, 315, L321

Padgett, D. L., Strom, S. E., \& Ghez, A. 1997, ApJ, 477, 705

Palla, F., \& Stahler, S. W. 1999, ApJ, 525, 772

Persi, P., Marenzi, A. R., Gómez, M., \& Olofsson, G. 2003, A\&A, 399, 995

Porras, A., Jorgensen, J., \& Allen, L. E. 2007, ApJ, 656, 493
Prusti, T., Whittet, D. C. B., Assendorp, R., \& Wesselius, P. R. 1992, A\&A, 260,151

Rebull, L., et al. 2007, ApJS, 171, 447

Reipurth, B. 1994, A General Catalogue of Herbig-Haro Objects

Reipurth, B., \& Bally, J. 2001, ARA\&A, 39, 403

Robitaille, T. P., Whitney, B. A., Indebetow, R., \& Wood, K. 2007, ApJS, 169, 328 (R07)

Robitaille, T. P., et al. 2006, ApJS, 167, 256 (R06)

Rowan-Robinson, M., et al. 2004, MNRAS, 351, 1290

Rydgren, A. E. 1980, AJ, 85, 444

Sandell, G., et al. 1987, A\&A, 182, 237

Schwartz, R. D. 1977, ApJS, 35, 161 - 1991, ESO Rep. 11, 93

Spezzi, L., et al. 2007a, A\&A, 470, 281 (S07a) 2007b, ApJ, submitted (S07b)

Steenman, H., \& Thi, P. S. 1989, Ap\&SS, 161, 99

Tachihara, K., et al. 1996, PASJ, 48, 489

Teixeira, R., et al. 2000, A\&A, 361, 1143

Vrba, F. J., \& Rydgren, A. E. 1984, ApJ, 283, 123

Vuong, M. H., Cambrésy, L., \& Epchtein, N. 2001, A\&A, 379, 208

Weingartner, J. C., \& Draine, B. T. 2001, ApJ, 548, 296

Whittet, D. C. B., Laureijs, R. J., \& Zhang, C. Y. 1991, A\&A, 251, 524

Whittet, D. C. B., et al. 1987, MNRAS, 224, 497 1994, MNRAS, 268, 1 1997, A\&A, 327, 1194

Young, K. E., et al. 2005, ApJ, 628, 283

Zacharias, N., et al. 2004, BAAS, 36, 1418

Zuckerman, B. 2001, ARA\&A, 39, 549 